\title{
DIMENSION DE HEITMANN DES TREILLIS DISTRIBUTIFS ET DES ANNEAUX COMMUTATIFS
}

\author{
par \\ Thierry Coquand, Henri Lombardi \& Claude Quitté
}

\begin{abstract}
Résumé. - Nous étudions la notion de dimension introduite par Heitmann dans son article remarquable de 1984 [15], ainsi qu'une notion voisine, seulement implicite dans ses preuves. Nous développons ceci d'abord dans le cadre général de la théorie des treillis distributifs et des espaces spectraux. Nous obtenons ensuite des versions constructives de certains théorèmes importants d'algèbre commutative. Les versions constructives de ce ces théorèmes s'avèrent en fin de compte plus simples, et parfois plus générales, que les versions classiques abstraites correspondantes.
\end{abstract}

\begin{abstract}
Heitmann dimension of distributive lattices and commutative rings)
We study the notion of dimension which was introduced by R. Heitmann in his remarkable paper in 1984 [15]. We study also a related notion which was only implicit in his paper. First we do the job in the general framework of distributive lattices ans spectral spaces. Next we apply this to commutative algebra. This allows us to get constructive versions of important theorems. Constructive versions of these theorems are more simple, and sometimes more general than the abstract ones which have been obtained in classical mathematics.
\end{abstract}

Table des matières

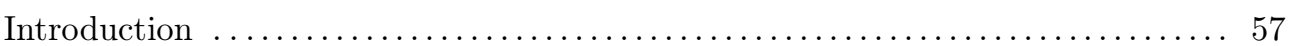

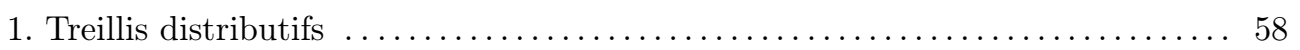

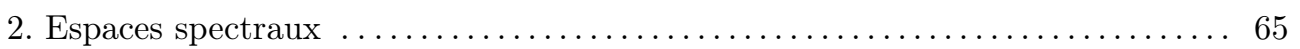

3. Dimensions de Krull et de Heitmann pour un treillis distributif . . . . ...... 73

4. Dimensions de Krull et Heitmann pour un anneau commutatif . . . . . . . . 82

5. Le théorème de Kronecker et le stable range de Bass

(versions non nothériennes de Heitmann) . . . . . . . . . . . . . . . . . 90

6. Le splitting off de Serre et le théorème de Forster,

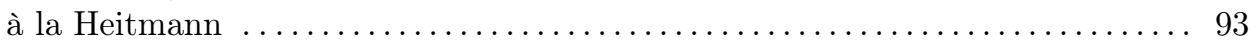

7. Théorèmes de Serre et de Swan, non noethériens $\ldots \ldots \ldots \ldots \ldots \ldots \ldots \ldots \ldots$

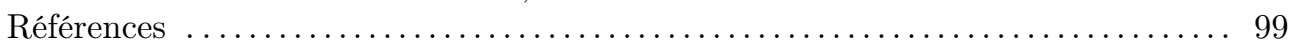

\section{Introduction}

Nous étudions la notion de dimension introduite par Heitmann dans son article de 1984 [15], ainsi qu'une notion voisine, seulement implicite dans ses preuves. Nous développons ceci d'abord dans le cadre général de la théorie des treillis distributifs et des espaces spectraux. Nous appliquons ensuite cette problématique dans le cadre de l'algèbre commutative.

Dans la dualité entre treillis distributifs et espaces spectraux, le spectre de Zariski d'un anneau commutatif correspond (comme l'a indiqué Joyal [18]) au treillis des idéaux qui sont radicaux

Classification mathématique par sujets (2000). — 13C15, 03F65, 13A15, $13 \mathrm{E} 05$.

Mots clefs. - Dimension de Krull, Dimension de Heitmann, Bord d'une sous variété, Théorème « Stable range * de Bass, Théorème « Splitting off » de Serre, Théorème de Forster-Swan, Théorème de Kronecker, Nombre de générateurs d'un module, Mathématiques constructives. 
d'idéaux de type fini. Nous montrons que l'espace spectral défini par Heitmann pour sa notion de dimension correspond au treillis formé par les idéaux qui sont radicaux de Jacobson d'idéaux de type fini. Ceci nous permet d'obtenir une définition constructive élémentaire de la dimension définie par Heitmann (que nous notons Jdim). Nous introduisons une autre dimension, que nous appelons dimension de Heitmann (et que nous notons Hdim), qui est «meilleure » en ce sens que Hdim $\leq$ Jdim et qu'elle permet des preuves par récurrence naturelles.

Nous obtenons alors des versions constructives de certains théorèmes classiques importants, dans leur version non nœthérienne (en général due à Heitmann).

Les versions constructives de ce ces théorèmes s'avèrent en fin de compte plus simples, et parfois plus générales, que les versions classiques abstraites correspondantes.

En particulier nous rappelons les versions non nœethériennes des théorèmes de Swan et de Serre (splitting off) obtenues récemment pour la première fois dans [5].

Naturellement, le principal avantage que nous voyons dans notre traitement est son caractère tout à fait élémentaire. En particulier nous n'utilisons pas d'hypothèses « non nécessaires » comme l'axiome du choix et le principe du tiers exclu, inévitables pour faire fonctionner les preuves classiques antérieures.

Enfin, le fait de s'être débarrassé de toute hypothèse nœthérienne est aussi non négligeable, et permet de mieux voir l'essence des choses.

En conclusion cet article peut être vu pour l'essentiel comme une mise au point constructive de la théorie des espaces spectraux via celle des treillis distributifs, avec une insistance particulière sur la dimension de Heitmann et quelques applications marquantes en algèbre commutative.

Remarque. - Nous avons résolu de la manière suivante un problème de terminologie qui se pose en rédigeant cet article. Le mot «dualité » apparait a priori dans le contexte des treillis distributifs avec deux significations différentes. Il y a d'une part la dualité qui correspond au renversement de la relation d'ordre dans un treillis. D'autre part il y a la dualité entre treillis distributifs et espaces spectraux, qui correspond à une antiéquivalence de catégorie. Nous avons décidé de réserver « dualité » pour ce dernier usage. Le terme « treillis dual » a donc été systématiquement remplacé par « treillis opposé ». De même on a remplacé « la notion duale » par « la notion renversée » ou par « la notion opposée », et «par dualité » par «par renversement de l'ordre ».

\section{Treillis distributifs}

Les axiomes des treillis distributifs peuvent être formulés avec des égalités universelles concernant uniquement les deux lois $\wedge$ et $\vee$ et les deux constantes $0_{\mathbf{T}}$ (l'élément minimum du treillis distributif $\mathbf{T}$ ) et $1_{\mathbf{T}}$ (le maximum). La relation d'ordre est alors définie par $a \leq_{\mathbf{T}} b \Leftrightarrow a \wedge b=a$. On obtient ainsi une théorie purement équationnelle, avec toutes les facilités afférentes. Par exemple on peut définir un treillis distributif par générateurs et relations, la catégorie comporte des limites inductives (qu'on peut définir par générateurs et relations) et des limites projectives (qui ont pour ensembles sous-jacents les limites projectives ensemblistes correspondantes).

Un ensemble totalement ordonné est un treillis distributif s'il possède un maximum et un minimum. On note $\mathbf{n}$ un ensemble totalement ordonné à $n$ éléments, c'est un treillis distributif si $n \neq 0$. Le treillis $\mathbf{2}$ est le treillis distributif libre à 0 générateur, et $\mathbf{3}$ celui à un générateur.

Pour tout treillis distributif $\mathbf{T}$, si on remplace la relation d'ordre $x \leq_{\mathbf{T}} y$ par la relation symétrique $y \leq_{\mathbf{T}} x$ on obtient le treillis opposé $\mathbf{T}^{\circ}$ avec échange de $\wedge$ et $\vee$ (on dit parfois treillis dual). 
1.1. Idéaux, filtres. $-\mathrm{Si} \varphi: \mathbf{T} \rightarrow \mathbf{T}^{\prime}$ est un morphisme de treillis distributifs, $\varphi^{-1}(0)$ est appelé un idéal de $T$. Un idéal $\mathfrak{I}$ de $\mathbf{T}$ est une partie de $\mathbf{T}$ soumise aux contraintes suivantes :

$$
\left.\begin{array}{rll}
x, y \in \mathfrak{I} & \Longrightarrow & 0 \in \mathfrak{I} \\
x \vee y \in \mathfrak{I} \\
x \in \mathfrak{I}, z \in \mathbf{T} & \Longrightarrow & x \wedge z \in \mathfrak{I}
\end{array}\right\}
$$

(la dernière se réécrit $(x \in \mathfrak{I}, y \leq x) \Rightarrow y \in \mathfrak{I})$. Un idéal principal est un idéal engendré par un seul élément $a:$ il est égal à

$$
\downarrow a=\{x \in \mathbf{T} \mid x \leq a\}
$$

L'idéal $\downarrow a$, muni des lois $\wedge$ et $\vee$ de $\mathbf{T}$ est un treillis distributif dans lequel l'élément maximum est a. L'injection canonique $\downarrow a \rightarrow \mathbf{T}$ n'est pas un morphisme de treillis distributifs parce que l'image de $a$ n'est pas égale à $1_{\mathbf{T}}$. Par contre l'application surjective $\mathbf{T} \rightarrow \downarrow a, x \mapsto x \wedge a$ est un morphisme surjectif, qui définit donc $\downarrow a$ comme une structure quotient.

La notion opposée à celle d'idéal est la notion de filtre. Le filtre principal engendré par $a$ est noté $\uparrow a$.

L'idéal engendré par une partie $J$ de $\mathbf{T}$ est

$$
\mathcal{I}_{\mathbf{T}}(J)=\left\{x \in \mathbf{T} \mid \exists J_{0} \in \mathcal{P}_{\mathrm{f}}(J), x \leq \bigvee J_{0}\right\} .
$$

En conséquence tout idéal de type fini est principal.

Si $A$ et $B$ sont deux parties de $\mathbf{T}$ on note

$$
A \vee B=\{a \vee b \mid a \in A, b \in B\} \text { et } A \wedge B=\{a \wedge b \mid a \in A, b \in B\}
$$

Alors l'idéal engendré par deux idéaux $\mathfrak{a}$ et $\mathfrak{b}$ est égal à

$$
\mathcal{I}_{\mathbf{T}}(\mathfrak{a} \cup \mathfrak{b})=\mathfrak{a} \vee \mathfrak{b}=\{z \mid \exists x \in \mathfrak{a}, \exists y \in \mathfrak{b}, z \leq x \vee y\} .
$$

L'ensemble des idéaux de $\mathbf{T}$ forme lui même un treillis distributif pour l'inclusion, avec pour inf de $\mathfrak{a}$ et $\mathfrak{b}$ l'idéal :

$$
\mathfrak{a} \cap \mathfrak{b}=\mathfrak{a} \wedge \mathfrak{b} .
$$

Ainsi les opérations $\vee$ et $\wedge$ définies en (3) correspondent au sup et au inf dans le treillis des idéaux.

On notera $\mathcal{F}_{\mathbf{T}}(S)$ le filtre de $\mathbf{T}$ engendré par le sous ensemble $S$. Quand on considère le treillis des filtres il faut faire attention à ce que produit le renversement de la relation d'ordre $: \mathfrak{f} \cap \mathfrak{g}=\mathfrak{f} \vee \mathfrak{g}$ est le inf de $\mathfrak{f}$ et $\mathfrak{g}$, tandis que leur sup est égal à $\mathcal{F}_{\mathbf{T}}(\mathfrak{f} \cup \mathfrak{g})=\mathfrak{f} \wedge \mathfrak{g}$.

Le treillis quotient de $\mathbf{T}$ par l'idéal $\mathfrak{J}$, noté $\mathbf{T} /(\mathfrak{J}=0)$ est défini comme le treillis distributif engendré par les éléments de $\mathbf{T}$ avec pour relations, les relations vraies dans $\mathbf{T}$ d'une part, et les relations $x=0$ pour les $x \in \mathfrak{J}$ d'autre part. Il peut aussi être défini par la relation de préordre

$$
a \preceq b \quad \Longleftrightarrow \quad a \leq_{\mathbf{T} /(\mathfrak{J}=0)} b \quad \stackrel{\text { def }}{\Longleftrightarrow} \exists x \in \mathfrak{J} \quad a \leq x \vee b
$$

Ceci donne

$$
a \equiv b \quad \bmod (\mathfrak{J}=0) \quad \Longleftrightarrow \quad \exists x \in \mathfrak{J} \quad a \vee x=b \vee x
$$

et dans le cas du quotient par un idéal principal $\downarrow a$ on obtient $\mathbf{T} /(a=0) \simeq \uparrow a$ avec le morphisme $y \mapsto y \vee a$ de $\mathbf{T}$ vers $\uparrow a$.

Transporteur, différence. - Par analogie avec l'algèbre commutative, si $\mathfrak{b}$ est un idéal et $A$ une partie de $\mathbf{T}$ on notera

$$
\mathfrak{b}: A \stackrel{\text { def }}{=}\{x \in \mathbf{T} \mid \forall a \in A \quad a \wedge x \in \mathfrak{b}\}
$$

Si $\mathfrak{a}$ est l'idéal engendré par $A$ on $\mathfrak{a} \mathfrak{b}: A=\mathfrak{b}: \mathfrak{a}$, on l'appelle le transporteur de $\mathfrak{a}$ dans $\mathfrak{b}$.

On note aussi $b: a$ l'idéal $(\downarrow b):(\downarrow a)=\{x \in \mathbf{T}|x \in \mathbf{T}| x \wedge a \leq b\}$. 
La notion opposée est celle de filtre différence de deux filtres

$$
\mathfrak{f} \backslash \mathfrak{f}^{\prime} \stackrel{\text { def }}{=}\left\{x \in \mathbf{T} \mid \forall a \in \mathfrak{f}^{\prime} \quad a \vee x \in \mathfrak{f}\right\}
$$

On note aussi $b \backslash a$ le filtre $(\uparrow b) \backslash(\uparrow a)=\{x \in \mathbf{T} \mid b \leq x \vee a\}$.

Radical de Jacobson. - Un idéal $\mathfrak{m}$ d'un treillis distributif $\mathbf{T}$ non trivial (i.e. distinct de 1) est dit maximal si $\mathbf{T} /(\mathfrak{m}=0)=\mathbf{2}$, c'est-à-dire si $1 \notin \mathfrak{m}$ et $\forall x \in \mathbf{T}(x \in \mathfrak{m}$ ou $\exists y \in \mathfrak{m} x \vee y=1)$.

Il revient au même de dire qu'il s'agit d'un idéal « maximal parmi les idéaux stricts ».

En mathématiques classiques on a le lemme suivant.

Lemme 1.1. - Dans un treillis distributif $\mathbf{T} \neq \mathbf{1}$ l'intersection des idéaux maximaux est égale à l'idéal

$$
\{a \in \mathbf{T} \mid \forall x \in \mathbf{T}(a \vee x=1 \Rightarrow x=1)\} .
$$

On l'appelle le radical de Jacobson de $\mathbf{T}$. On le note $\mathrm{J}_{\mathbf{T}}(0)$.

Plus généralement l'intersection des idéaux maximaux contenant un idéal strict $\mathfrak{J}$ est égale à l'idéal

$$
\mathrm{J}_{\mathbf{T}}(\mathfrak{J})=\{a \in \mathbf{T} \mid \forall x \in \mathbf{T}(a \vee x=1 \Rightarrow \exists z \in \mathfrak{J} \quad z \vee x=1)\}
$$

On l'appelle le radical de Jacobson de l'idéal $\mathfrak{J}$. En particulier :

$$
\mathrm{J}_{\mathbf{T}}(\downarrow b)=\{a \in \mathbf{T} \mid \forall x \in \mathbf{T}(a \vee x=1 \Rightarrow b \vee x=1)\}
$$

Démonstration. - La deuxième affirmation résulte de la première en passant au treillis quotient $\mathbf{T} /(\mathfrak{J}=0)$. Voyons la première. On montre que $a$ est en dehors d'au moins un idéal maximal si et seulement si $\exists x \neq 1$ tel que $a \vee x=1$. Si c'est le cas, un idéal maximal qui contient $x$ (il en existe puisque $x \neq 1$ ) ne peut pas contenir $a$ car il contiendrait $a \vee x$. Inversement, si $\mathfrak{m}$ est un idéal maximal ne contenant pas $a$, l'idéal engendré par $\mathfrak{m}$ et $a$ contient 1 . Or cet idéal est l'ensemble des éléments majorés par au moins un $a \vee x$ où $x$ parcourt $\mathfrak{m}$.

En mathématiques classiques un treillis distributif est appelé treillis de Jacobson si tout idéal premier est égal à son radical de Jacobson. Comme tout idéal est intersection des idéaux premiers qui le contiennent, cela implique que tout idéal est égal à son radical de Jacobson.

En mathématiques constructives on adopte les définitions suivantes.

Définitions 1.2. - (radical de Jacobson, treillis faiblement Jacobson)

1. Si $\mathfrak{J}$ est un idéal de $\mathbf{T}$ son radical de Jacobson est défini par l'égalité (8) (on ne fait pas l'hypothèse que $\mathbf{T} \neq \mathbf{1})$. On notera $\mathrm{J}_{\mathbf{T}}(a)$ pour $\mathrm{J}_{\mathbf{T}}(\downarrow a)$.

2. Un treillis distributif est appelé un treillis faiblement Jacobson si tout idéal principal est égal à son radical de Jacobson, c'est-à-dire encore

$$
\forall a, b \in \mathbf{T}[(\forall x \in 1 \backslash a, b \vee x=1) \Rightarrow a \leq b]
$$

On vérifie sans difficulté que $\mathrm{J}_{\mathbf{T}}(\mathfrak{J})$ est un idéal et que $1 \in \mathrm{J}_{\mathbf{T}}(\mathfrak{J})$ si et seulement si $1 \in \mathfrak{J}$.

1.2. Quotients. — Un treillis distributif quotient $\mathbf{T}^{\prime}$ de $\mathbf{T}$ est donné par une relation binaire $\preceq$ sur $\mathbf{T}$ vérifiant les propriétés suivantes :

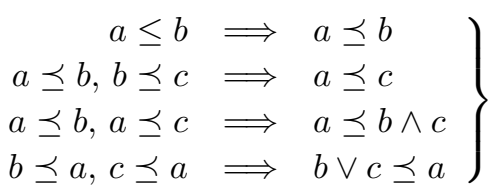

Proposition 1.3. - Soit $\mathbf{T}$ un treillis distributif et $(J, U)$ un couple de parties de $\mathbf{T}$. On considère le quotient $\mathbf{T}^{\prime}$ de $\mathbf{T}$ défini par les relations $x=0$ pour les $x \in J$ et $y=1$ pour les $y \in U$. Alors on a $a \leq_{\mathbf{T}^{\prime}} b$ si et seulement si il existe une partie finie $J_{0}$ de $J$ et une partie finie $U_{0}$ de $U$ telles que :

$$
a \wedge \bigwedge U_{0} \leq_{\mathbf{T}} b \vee \bigvee J_{0}
$$


Nous noterons $\mathbf{T} /(J=0, U=1)$ ce treillis quotient $\mathbf{T}^{\prime}$.

Idéaux dans un quotient. — Le fait suivant résulte des égalités (1), (3), (4) et (5).

Fait 1.4. - Soit $\pi: \mathbf{T} \rightarrow \mathbf{L}$ un treillis quotient.

- L'image réciproque d'un idéal de $\mathbf{L}$ par $\pi^{-1}$ est un idéal de $\mathbf{T}$, ceci donne un morphisme pour $\vee$ et $\wedge$ (mais pas nécessairement pour 0 et 1$)$.

- L'image d'un idéal de $\mathbf{T}$ par $\pi$ est un idéal de $\mathbf{L}$, ceci donne un homomorphisme surjectif de treillis.

- Un idéal de $\mathbf{T}$ est de la forme $\pi^{-1}(\mathfrak{a})$ si et seulement si il est saturé pour la relation $=_{\mathbf{L}}$.

- Résultats analogues pour les filtres.

Notez qu'en algèbre commutative, le morphisme de passage au quotient par un idéal ne se comporte pas aussi bien pour les idéaux dans le cas d'une intersection puisqu'on peut très bien avoir $\mathfrak{a}+(\mathfrak{b} \cap \mathfrak{c}) \varsubsetneqq(\mathfrak{a}+\mathfrak{b}) \cap(\mathfrak{a}+\mathfrak{c})$.

Le lemme suivant donne quelques renseignements complémentaires pour les quotients par un idéal et par un filtre.

Lemme 1.5. - Soit $\mathfrak{a}$ un idéal et $\mathfrak{f}$ un filtre de $\mathbf{T}$

1. Si $\mathbf{L}=\mathbf{T} /(\mathfrak{a}=0)$ alors la projection canonique $\pi: \mathbf{T} \rightarrow \mathbf{L}$ établit une bijection croissante entre les idéaux de $\mathbf{T}$ contenant $\mathfrak{a}$ et les idéaux de $\mathbf{L}$. La bijection réciproque est fournie par $\mathfrak{j} \mapsto \pi^{-1}(\mathfrak{j})$. En outre si $\mathfrak{j}$ est un idéal de $\mathbf{L}, \pi^{-1}\left(\mathrm{~J}_{\mathbf{L}}(\mathfrak{j})\right)=\mathrm{J}_{\mathbf{T}}\left(\pi^{-1}(\mathfrak{j})\right)$.

2. Si $\mathbf{L}=\mathbf{T} /(\mathfrak{f}=1)$ alors la projection canonique $\pi: \mathbf{T} \rightarrow \mathbf{L}$ établit une bijection croissante entre les idéaux $\mathfrak{J}$ de $\mathbf{T}$ vérifiant $« \forall \in \mathfrak{f}, \mathfrak{J}: f=\mathfrak{J} »$ et les idéaux de $\mathbf{L}$.

Remarque. - On notera que $\mathbf{T} \mapsto \mathrm{J}_{\mathbf{T}}(0)$ n'est pas une opération fonctorielle. La deuxième affirmation du point $1 \mathrm{du}$ lemme précédent, qui admet une preuve constructive directe, s'explique facilement en mathématiques classiques par le fait que, dans le cas très particulier du quotient par un idéal, les idéaux maximaux de $\mathbf{L}$ contenant $\mathbf{j}$ correspondent par $\pi^{-1}$ aux idéaux maximaux de $\mathbf{T}$ contenant $\pi^{-1}(\mathfrak{j})$.

Recollement de treillis quotients. - En algèbre commutative, si $\mathfrak{a}$ et $\mathfrak{b}$ sont deux idéaux d'un anneau $\mathbf{A}$ on a une « suite exacte $»$ de $\mathbf{A}$-modules (avec $j$ et $p$ des homomorphismes d'anneaux)

$$
0 \rightarrow \mathbf{A} /(\mathfrak{a} \cap \mathfrak{b}) \stackrel{j}{\longrightarrow}(\mathbf{A} / \mathfrak{a}) \times(\mathbf{A} / \mathfrak{b}) \stackrel{p}{\longrightarrow} \mathbf{A} /(\mathfrak{a}+\mathfrak{b}) \rightarrow 0
$$

qu'on peut lire en langage courant : le système de congruences $x \equiv a \bmod \mathfrak{a}, x \equiv b \bmod \mathfrak{b}$ admet une solution si et seulement si $a \equiv b \bmod \mathfrak{a}+\mathfrak{b}$ et dans ce cas la solution est unique modulo $\mathfrak{a} \cap \mathfrak{b}$. Il est remarquable que ce « théorème des restes chinois » se généralise à un système quelconque de congruences si et seulement si l'anneau est arithmétique, c'est-à-dire si le treillis des idéaux est distributif (le théorème des restes chinois « contemporain » concerne le cas particulier d'une famille d'idéaux deux à deux comaximaux, et il fonctionne sans hypothèse sur l'anneau de base).

D'autres épimorphismes de la catégorie des anneaux commutatifs sont les localisations. Et il y a un principe de recollement analogue au théorème des restes chinois pour les localisations, extrêmement fécond (le principe local global).

De la même manière on peut récupérer un treillis distributif à partir d'un nombre fini de ses quotients, si l'information qu'ils contiennent est «suffisante ». On peut voir ceci au choix comme une procédure de recollement (de passage du local au global), ou comme une version du théorème des restes chinois pour les treillis distributifs. Voyons les choses plus précisément.

Définition 1.6. - Soit $\mathbf{T}$ un treillis distributif, $\left(\mathfrak{a}_{i}\right)_{i=1, \ldots n}\left(\operatorname{resp} .\left(\mathfrak{f}_{i}\right)_{i=1, \ldots n}\right)$ une famille finie d'idéaux (resp. de filtres) de $\mathbf{T}$. On dit que les idéaux $\mathfrak{a}_{i}$ recouvrent $\mathbf{T}$ si $\bigcap_{i} \mathfrak{a}_{i}=\{0\}$. De même on dit que les filtres $\mathfrak{f}_{i}$ recouvrent $\mathbf{T}$ si $\bigcap_{i} \mathfrak{f}_{i}=\{1\}$. 
Pour un idéal $\mathfrak{b}$ nous écrivons $x \equiv y \bmod \mathfrak{b}$ comme abbréviation pour $x \equiv y \quad \bmod (\mathfrak{b}=0)$. Le fait suivant est facile.

Fait 1.7. - Soit $\mathbf{T}$ un treillis distributif, $\left(\mathfrak{a}_{i}\right)_{i=1, \ldots n}$ une famille finie d'idéaux de $\mathbf{T}$ et $\mathfrak{a}=\bigcap_{i} \mathfrak{a}_{i}$.

1. Si $\left(x_{i}\right)$ est une famille d'éléments de $\mathbf{T}$ telle que pour chaque $i<j$ on a $x_{i} \equiv x_{j} \bmod \mathfrak{a}_{i} \vee \mathfrak{a}_{j}$, alors il existe un unique $x$ modulo $\mathfrak{a}$ vérifiant $: x \equiv x_{i} \bmod \mathfrak{a}_{i}(i=1, \ldots, n)$.

2. Notons $\mathbf{T}_{i}=\mathbf{T} /\left(\mathfrak{a}_{i}=0\right), \mathbf{T}_{i j}=\mathbf{T}_{j i}=\mathbf{T} /\left(\mathfrak{a}_{i} \cup \mathfrak{a}_{j}=0\right), \pi_{i}: \mathbf{T} \rightarrow \mathbf{T}_{i}$ et $\pi_{i j}: \mathbf{T}_{i} \rightarrow \mathbf{T}_{i j}$ les projections canoniques. Si les $\mathfrak{a}_{i}$ recouvrent $\mathbf{T},\left(\mathbf{T},\left(\pi_{i}\right)_{i=1, \ldots n}\right)$ est la limite projective du diagramme

$$
\left(\left(\mathbf{T}_{i}\right)_{1 \leq i \leq n},\left(\mathbf{T}_{i j}\right)_{1 \leq i<j \leq n} ;\left(\pi_{i j}\right)_{1 \leq i \neq j \leq n}\right) .
$$

3. Soit maintenant $\left(\mathfrak{f}_{i}\right)_{i=1, \ldots n}$ une famille finie de filtres, notons $\mathbf{T}_{i}=\mathbf{T} /\left(\mathfrak{f}_{i}=1\right), \mathbf{T}_{i j}=\mathbf{T}_{j i}=$ $\mathbf{T} /\left(\mathfrak{f}_{i} \cup \mathfrak{f}_{j}=1\right), \pi_{i}: \mathbf{T} \rightarrow \mathbf{T}_{i}$ et $\pi_{i j}: \mathbf{T}_{i} \rightarrow \mathbf{T}_{i j}$ les projections canoniques. Si les $\mathfrak{f}_{i}$ recouvrent $\mathbf{T},\left(\mathbf{T},\left(\pi_{i}\right)_{i=1, \ldots n}\right)$ est la limite projective du diagramme

$$
\left(\left(\mathbf{T}_{i}\right)_{1 \leq i \leq n},\left(\mathbf{T}_{i j}\right)_{1 \leq i<j \leq n} ;\left(\pi_{i j}\right)_{1 \leq i \neq j \leq n}\right) .
$$

Il y a aussi une procédure de recollement proprement dit, qui fonctionne avec des quotients arbitraires.

Proposition 1.8. - (recollement abstrait de treillis)

Soit $\left(\left(\mathbf{T}_{i}\right)_{1 \leq i \leq n},\left(\mathbf{T}_{i j}\right)_{1 \leq i \neq j \leq n} ;\left(\pi_{i j}\right)_{1 \leq i \neq j \leq n}\right)$ un diagramme de treillis distributifs avec $\mathbf{T}_{j i}=\mathbf{T}_{j i}$, et les $\pi_{i j}: \mathbf{T}_{i} \rightarrow \mathbf{T}_{i j}$ pour $i \neq j$ qui sont des homomorphismes surjectifs. Supposons que les conditions de compatibilité naturelle pour « les quotients par triples » soient vérifiées : si $i<j<k$, $x_{i} \in \mathbf{T}_{i}, x_{j} \in \mathbf{T}_{j}, x_{k} \in \mathbf{T}_{k}$, alors parmi les trois relations $\pi_{i j}\left(x_{i}\right)=\pi_{j i}\left(x_{j}\right), \pi_{i k}\left(x_{i}\right)=\pi_{k i}\left(x_{k}\right)$, $\pi_{j k}\left(x_{j}\right)=\pi_{k j}\left(x_{k}\right)$, deux impliquent la troisième. Alors la limite projective du diagramme est donnée par un treillis distributif $\mathbf{T}$ et par des morphismes $\pi_{i}: \mathbf{T} \rightarrow \mathbf{T}_{i}$ qui identifient les $\mathbf{T}_{i}$ à un système de quotients de $\mathbf{T}$.

Démonstration. - Il faut essentiellement vérifier que les homomorphismes $\mathbf{T} \rightarrow \mathbf{T}_{i}$ sont surjectifs. Soit par exemple $x_{1} \in \mathbf{T}_{1}$. Pour $i>1$ il existe $x_{i} \in \mathbf{T}_{i}$ tel que $\pi_{1 i}\left(x_{1}\right)=\pi_{i 1}\left(x_{i}\right)$, ceci parce que les $\pi_{i 1}$ sont surjectifs. Et la famille $\left(x_{i}\right)$ ainsi obtenue est bien un élément de la limite projective, vue la condition de compatibilité "par triples".

$\mathrm{Au}$ vu de ce « recollement abstrait », on voit que le fait 1.7 peut s'interpréter en disant que dans le cas des idéaux et dans celui des filtres tout recouvrement fini est un recollement.

Treillis de Heitmann. — Un quotient intéressant, qui n'est ni un quotient par un idéal ni un quotient par un filtre, est le treillis de Heitmann.

Lemme 1.9. - Sur un treillis distributif arbitraire $\mathbf{T}$ la relation $\mathrm{J}_{\mathbf{T}}(a) \subseteq \mathrm{J}_{\mathbf{T}}(b)$ est une relation de préordre $a \preceq b$ qui définit un quotient de $\mathbf{T}$. On a aussi :

$$
a \preceq b \quad a \in \mathrm{J}_{\mathbf{T}}(b) \Longleftrightarrow \forall x \in \mathbf{T}(a \vee x=1 \Rightarrow b \vee x=1)
$$

Démonstration. - Les équivalences $a \in \mathrm{J}_{\mathbf{T}}(b) \Leftrightarrow \mathrm{J}_{\mathbf{T}}(a) \subseteq \mathrm{J}_{\mathbf{T}}(b) \Leftrightarrow \forall x \in \mathbf{T}(a \vee x=1 \Rightarrow$ $b \vee x=1$ ) résultent de ce qui a été dit page 60 concernant le radical de Jacobson d'un idéal (voir l'égalité (9)).

Par ailleurs on vérifie sans difficulté les relations (11) nécessaires pour qu'un préordre définisse un quotient.

Définition 1.10. - On appelle treillis de Heitmann de $\mathbf{T}$ et on note $\mathrm{He}(\mathbf{T})$ le treillis quotient de $\mathbf{T}$ obtenu en remplaçant sur $\mathbf{T}$ la relation d'ordre $\leq_{\mathbf{T}}$ par la relation de préordre $\preceq_{\text {He( } \mathbf{T})}$ définie comme suit

$$
a \preceq \text { He(T) } b \quad \stackrel{\text { def }}{\Longleftrightarrow} \quad \mathrm{J}_{\mathbf{T}}(a) \subseteq \mathrm{J}_{\mathbf{T}}(b)
$$


Ce treillis quotient peut être identifié à l'ensemble des idéaux $\mathrm{J}_{\mathbf{T}}(a)$, avec la projection canonique

$$
\mathbf{T} \longrightarrow \mathrm{He}(\mathbf{T}), \quad a \longmapsto \mathrm{J}_{\mathbf{T}}(a)
$$

Dire que le treillis $\mathbf{T}$ est faiblement Jacobson revient à dire que $\mathbf{T}=\mathrm{He}(\mathbf{T})$.

Notez qu'avec l'identification précédente on a les égalités :

$$
\mathrm{J}_{\mathbf{T}}(a \wedge b)=\mathrm{J}_{\mathbf{T}}(a) \wedge_{\mathrm{He}(\mathbf{T})} \mathrm{J}_{\mathbf{T}}(b), \quad \mathrm{J}_{\mathbf{T}}(a \vee b)=\mathrm{J}_{\mathbf{T}}(a) \vee_{\mathrm{He}(\mathbf{T})} \mathrm{J}_{\mathbf{T}}(b)
$$

Le lemme suivant est une précision (et une généralisation) de la première égalité ci-dessus. Il nous sera utile dans la suite.

Lemme 1.11. - Si $\mathfrak{a}$ et $\mathfrak{b}$ sont deux idéaux de $\mathbf{T}$, on a $\mathrm{J}_{\mathbf{T}}(\mathfrak{a} \cap \mathfrak{b})=\mathrm{J}_{\mathbf{T}}(\mathfrak{a}) \cap \mathrm{J}_{\mathbf{T}}(\mathfrak{b})$.

Démonstration. - Il suffit de montrer que si $z \in \mathrm{J}_{\mathbf{T}}(\mathfrak{a}) \cap \mathrm{J}_{\mathbf{T}}(\mathfrak{b})$ alors $z \in \mathrm{J}_{\mathbf{T}}(\mathfrak{a} \cap \mathfrak{b})$. Soit $t \in \mathbf{T}$ tel que $z \vee t=1$, nous cherchons $c \in \mathfrak{a} \cap \mathfrak{b}$ tel que $c \vee t=1$. Or nous avons un $a \in \mathfrak{a}$ tel que $a \vee t=1$ et un $b \in \mathfrak{b}$ tel que $b \vee t=1$. Il suffit donc de prendre $c=a \wedge b$.

On notera que la preuve ne marcherait pas pour une intersection infinie d'idéaux.

Fait 1.12. - Soit $\mathbf{T}$ un treillis distributif, $\mathbf{T}^{\prime}=\mathbf{T} /\left(\mathrm{J}_{\mathbf{T}}(0)=0\right), x \in \mathbf{T}$ et $\mathfrak{a}$ un idéal.

1. $x={ }_{\mathrm{He}(\mathbf{T})} 1 \Longleftrightarrow x=1$.

2. $x={ }_{\mathrm{He}(\mathbf{T})} 0 \Longleftrightarrow x \in \mathrm{J}_{\mathbf{T}}(0)$.

3. $\operatorname{He}(\operatorname{He}(\mathbf{T}))=\operatorname{He}\left(\mathbf{T}^{\prime}\right)=\operatorname{He}(\mathbf{T})$.

4. Si $\mathbf{L}=\mathbf{T} /(\mathfrak{a}=0)$, $\mathrm{He}(\mathbf{L})$ s'identifie à $\mathrm{He}(\mathbf{T}) /\left(\mathrm{J}_{\mathbf{T}}(\mathfrak{a})=0\right)$.

Remarque. - On notera cependant que He ne définit pas un foncteur.

Démonstration. - Les points 1 et 2 sont immédiats.

Le point 4 est laissé au lecteur. Il implique $\mathrm{He}\left(\mathbf{T}^{\prime}\right)=\mathrm{He}(\mathbf{T})$.

Dans le point $3 \mathrm{He}(\mathrm{He}(\mathbf{T}))$ et $\mathrm{He}\left(\mathbf{T}^{\prime}\right)$ sont identifiés à des quotients de $\mathbf{T}$. Montrons que $\mathrm{He}(\mathbf{T})=$ $\mathrm{He}(\mathrm{He}(\mathbf{T}))$, c'est-à-dire que pour $a, b \in \mathbf{T}, a \preceq_{\mathrm{He}(\mathrm{He}(\mathbf{T}))} b \Rightarrow a \preceq_{\mathrm{He}(\mathbf{T})} b$. Par définition l'hypothèse signifie $: \forall x \in \mathbf{T}\left(a \vee x=\mathrm{He(T)} 1 \Rightarrow b \vee x==_{\mathrm{He}(\mathbf{T})} 1\right)$. Or d'après le point 1 cela veut dire $\forall x \in \mathbf{T}(a \vee x=1 \Rightarrow b \vee x=1)$, c'est-à-dire $a \preceq \operatorname{He(T)} b$.

1.3. Algèbres de Heyting, de Brouwer, de Boole. — Un treillis distributif $\mathbf{T}$ est appelé un treillis implicatif [8] ou une algèbre de Heyting [17] lorsqu'il existe une opération binaire $\rightarrow$ vérifiant pour tous $a, b, c$ :

$$
a \wedge b \leq c \Longleftrightarrow a \leq(b \rightarrow c)
$$

Ceci signifie que pour tous $b, c \in \mathbf{T}$, l'idéal $c: b$ est principal, son générateur étant noté $b \rightarrow c$. Donc si elle existe, l'opération $\rightarrow$ est déterminée de manière unique par la structure du treillis. On définit alors la loi unaire $\neg x=x \rightarrow 0$. La structure d'algèbre de Heyting peut être définie comme purement équationnelle en donnant de bons axiomes. Précisément un treillis $\mathbf{T}$ (non supposé distributif) muni d'une loi $\rightarrow$ est une algèbre de Heyting si et seulement si les axiomes suivants sont vérifiés (cf. [17]) :

$$
\begin{aligned}
a \rightarrow a & =1 \\
a \wedge(a \rightarrow b) & =a \wedge b \\
b \wedge(a \rightarrow b) & =b \\
a \rightarrow(b \wedge c) & =(a \rightarrow b) \wedge(a \rightarrow c)
\end{aligned}
$$

Notons aussi les faits importants suivants :

$$
\begin{aligned}
(a \vee b) \rightarrow c & =(a \rightarrow c) \wedge(b \rightarrow c) \\
\neg(a \vee b) & =\neg a \wedge \neg b \\
\neg \neg a & \leq a \\
\neg a \vee b & \leq a \rightarrow b \\
a \leq b & \Leftrightarrow a \rightarrow b=1
\end{aligned}
$$


Tout treillis distributif fini est une algèbre de Heyting, car tout idéal de type fini est principal.

Un cas particulier important d'algèbre de Heyting est une algèbre de Boole : c'est un treillis distributif dans lequel tout élément $x$ possède un complément, c'est-à-dire un élément $y$ vérifiant $y \wedge x=0$ et $y \vee x=1$ ( $y$ est noté $\neg x$ et on a $a \rightarrow b=\neg a \vee b$ ).

Un homomorphisme d'algèbres de Heyting est un homomorphisme $\varphi: \mathbf{T} \rightarrow \mathbf{T}^{\prime}$ de treillis distributifs qui vérifie $\varphi(a \rightarrow b)=\varphi(a) \rightarrow \varphi(b)$ pour tous $a, b \in \mathbf{T}$.

Le fait suivant est immédiat.

Fait 1.13. - Soit $\pi: \mathbf{T} \rightarrow \mathbf{T}^{\prime}$ un homomorphisme de treillis distributifs. Supposons que $\mathbf{T}$ et $\mathbf{T}^{\prime}$ sont deux algèbres de Heyting et notons $a \preceq b$ pour $\varphi(a) \leq_{\mathbf{T}^{\prime}} \varphi(b)$. Alors $\pi$ est un homomorphisme d'algèbres de Heyting si et seulement si on a pour tous $a, a^{\prime}, b, b^{\prime} \in \mathbf{T}$ :

$$
a \preceq a^{\prime} \Rightarrow\left(a^{\prime} \rightarrow b\right) \preceq(a \rightarrow b) \quad \text { et } \quad b \preceq b^{\prime} \Rightarrow(a \rightarrow b) \preceq\left(a \rightarrow b^{\prime}\right)
$$

On a aussi :

Fait 1.14. - Si $\mathbf{T}$ est une algèbre de Heyting tout quotient $\mathbf{T} /(y=0)$ (c'est-à-dire tout quotient par un idéal principal) est aussi une algèbre de Heyting.

Démonstration. - Soit $\pi: \mathbf{T} \rightarrow \mathbf{T}^{\prime}=\mathbf{T} /(y=0)$ la projection canonique. On a $\pi(x) \wedge \pi(a) \leq_{\mathbf{T}^{\prime}}$ $\pi(b) \Leftrightarrow \pi(x \wedge a) \leq_{\mathbf{T}^{\prime}} \pi(b) \Leftrightarrow x \wedge a \leq b \vee y \Leftrightarrow x \leq a \rightarrow(b \vee y)$. Or $y \leq b \vee y \leq a \rightarrow(b \vee y)$, donc $\pi(x) \wedge \pi(a) \leq_{\mathbf{T}^{\prime}} \pi(b) \Leftrightarrow x \leq(a \rightarrow(b \vee y)) \vee y$, c'est-à-dire $\pi(x) \leq_{\mathbf{T}^{\prime}} \pi(a \rightarrow(b \vee y))$, ce qui montre que $\pi(a \rightarrow(b \vee y))$ vaut pour $\pi(a) \rightarrow \pi(b)$ dans $\mathbf{T}^{\prime}$.

Remarque. - La notion d'algèbre de Heyting est reminiscente de la notion d'anneau cohérent en algèbre commutative. En effet un anneau cohérent peut être caractérisé comme suit : l'intersection de deux idéaux de type fini est un idéal de type fini et le transporteur d'un idéal de type fini dans un idéal de type fini est un idéal de type fini. Si on « relit» ceci pour un treillis distributif en se rappelant que tout idéal de type fini est principal on obtient une algèbre de Heyting.

Remarque. - Tout treillis distributif $\mathbf{T}$ engendre une algèbre de Heyting de façon naturelle. Autrement dit on peut rajouter formellement un générateur pour tout idéal $b: c$. Mais si on part d'un treillis distributif qui se trouve être une algèbre de Heyting, l'algèbre de Heyting qu'il engendre est strictement plus grande. Prenons par exemple le treillis 3 qui est le treillis distributif libre à un générateur. L'algèbre de Heyting qu'il engendre est donc l'algèbre de Heyting libre à un générateur. Or celle-ci est infinie (cf. [17]). A contrario le treillis booléen engendré par $\mathbf{T}$ (cf. [2]) reste égal à $\mathbf{T}$ lorsque celui-ci est booléen.

Treillis avec négation. - Un treillis distributif possède une négation si pour tout $x$ l'idéal $(0: x)$ est principal, engendré par un élément que l'on note $\neg x$. Les règles suivantes sont immédiates.

$$
\begin{aligned}
& a \leq b \quad \Rightarrow \quad \neg b \leq \neg a \\
& a \leq \neg \neg a \quad, \quad \neg a=\neg \neg \neg a \\
& \neg(a \vee b)=\neg a \wedge \neg b \quad, \quad \neg a \vee \neg b \leq \neg(a \wedge b) \\
& \neg(x \vee \neg x)=0 \quad, \quad \neg \neg(x \vee \neg x)=1
\end{aligned}
$$

Si pour tout $a, \neg \neg a=a$, le treillis est une algèbre de Boole parce qu'alors $x \vee \neg x=1$.

Fait 1.15. - Si $\mathbf{T}$ possède une négation, notons $\mathrm{F}_{\min }(\mathbf{T})=\mathfrak{f}$ le filtre engendré par les $x \vee \neg x$. Alors $\mathrm{He}\left(\mathbf{T}^{\circ}\right)=(\mathbf{T} /(\mathfrak{f}=1))^{\circ}$, et ce treillis est une algèbre de Boole.

Démonstration. - Il est clair que $\neg x$ est un complément de $x$ dans $\mathbf{T} /(\mathfrak{f}=1)$, ce treillis est donc une algèbre de Boole. En présence de la négation, la relation $a \leq_{\mathrm{He}\left(\mathbf{T}^{\circ}\right)} b$ est équivalente à $\neg a \leq \neg b$ et ceci est facilement équivalent à $b \leq a \bmod (\mathfrak{f}=1)$.

Fait 1.16. - Si $\mathbf{T}$ est un treillis avec négation, le treillis $\mathbf{T}^{\circ}$ est faiblement Jacobson si et seulement si $\mathbf{T}$ est une algèbre de Boole. 
Démonstration. - En présence de négation, les équations (13) et (14) donnent pour $a \leq_{\mathrm{He}\left(\mathbf{T}^{\circ}\right)} b$ la condition équivalente $\neg b \leq \neg a$. Le treillis $\mathbf{T}^{\circ}$ est donc faiblement Jacobson si et seulement si $\neg b \leq \neg a$ implique $a \leq b$. En particulier on doit avoir $a=\neg \neg a$ pour tout $a$.

Algèbres de Brouwer. - Un treillis distributif tel que le treillis opposé est une algèbre de Heyting est appelé une algèbre de Brouwer. C'est un treillis distributif dans lequel tous les filtres $c \backslash b$ sont principaux. On note alors $c-b$ le générateur de $c \backslash b$.

En passant au treillis opposé le fait suivant dit la même chose que le fait 1.15.

Fait 1.17. - Si T possède un complément de Brouwer, c'est-à-dire si pour tout $x$ le filtre $(1 \backslash x)$ est principal (engendré par un élément qu'on note $1-x$ ), notons $\mathrm{I}_{\max }(\mathbf{T})=\mathfrak{a}$ l'idéal engendré par les $x \wedge(1-x)$. Alors $\mathrm{He}(\mathbf{T})$ est égal à l'algèbre de Boole $\mathbf{T} /(\mathfrak{a}=0)$.

Nous laissons au lecteur le soin de traduire le fait 1.16 .

1.4. Treillis distributifs nothériens. - En mathématiques classiques, pour un treillis distributif $\mathbf{T}$ les propriétés suivantes sont équivalentes :

(1) Tout idéal est principal.

(2) Toute suite croissante est stationnaire.

(3) Toute suite croissante d'idéaux est stationnaire.

Un tel treillis est appelé nœthérien (par analogie avec l'algèbre commutative, on pourrait l'appeler principal). C'est clairement est une algèbre de Heyting.

Tout sous treillis et tout treillis quotient d'un treillis nœthérien est nœthérien.

En mathématiques constructives la notion est plus délicate. Aucun treillis non trivial ne vérifie le point (2) (qui est a priori la formulation la plus faible des trois). On pourrait définir un treillis distributif nœethérien comme un treillis vérifiant une condition « ACC constructive d du style : toute suite croissante admet deux termes consécutifs égaux. Cette condition est équivalente à (2) en mathématiques classiques. Mais il y a a priori plusieurs variantes intéressantes.

En pratique, il semble qu'on est en général intéressé par le fait que certains idéaux bien précis sont principaux, comme dans le cas des algèbres de Heyting. Or le fait qu'un treillis est une algèbre de Heyting ne résulte pas constructivement de la condition ACC constructive (de la même manière, en algèbre commutative, la cohérence, qui est souvent plus importante que la nothérianité, ne résulte d'aucune variante constructive connue de la nothérianité).

Remarque. - Montrons en mathématiques classiques que si $\mathbf{T}$ et $\mathbf{T}^{\circ}$ sont nœethériens alors $\mathbf{T}$ est fini. Les idéaux maximaux sont des $\downarrow x$ où $x$ est un prédecesseur immédiat de 1 . Et le spectre maximal est fini, parce que si $\left(\mathfrak{m}_{n}\right)=\left(\downarrow x_{n}\right)$ est une suite infinie d'idéaux maximaux, la suite $\left(\bigwedge_{i \leq n} x_{i}\right)$ est strictement décroissante. On peut ensuite appliquer le résultat à chacun des treillis quotients par les idéaux maximaux. On termine par le lemme de König. Rendre cette preuve constructive, avec une définition constructive suffisamment forte de la nothériannité est un défi intéressant.

\section{Espaces spectraux}

\subsection{Généralités. -}

En mathématiques classiques. - Un idéal premier $\mathfrak{p}$ d'un treillis $\mathbf{T} \neq \mathbf{1}$ est un idéal dont le complémentaire $\mathfrak{f}$ est un filtre (qui est alors un filtre premier). On a alors $\mathbf{T} /(\mathfrak{p}=0, \mathfrak{f}=1) \simeq \mathbf{2}$. Il revient au même de se donner un idéal premier de $\mathbf{T}$ ou un morphisme de treillis distributifs $\mathbf{T} \rightarrow \mathbf{2}$.

Dans cette section, nous noterons $\theta_{\mathfrak{p}}: \mathbf{T} \rightarrow \mathbf{2}$ l'homomorphisme associé à l'idéal premier $\mathfrak{p}$.

On vérifie facilement que si $S$ est une partie génératrice du treillis distributif $\mathbf{T}$, un idéal premier $\mathfrak{p}$ de $\mathbf{T}$ est complètement caractérisé par sa trace sur $S$ (cf. [2]).

Un idéal maximal (resp. premier minimal) est un idéal maximal parmi les idéaux stricts (resp. minimal parmi les idéaux premiers). Il revient au même de dire que $\mathfrak{m}$ est maximal ou que le 
quotient $\mathbf{T} /(\mathfrak{m}=0) \simeq \mathbf{2}$, les idéaux maximaux sont donc premiers. Il revient au même de dire que $\mathfrak{p}$ est un idéal premier minimal ou que son complémentire est un filtre maximal.

En mathématiques classiques tout idéal strict est contenu dans un idéal maximal et (par renversement) tout filtre est contenu dans le complémentaire d'un idéal premier minimal.

Le spectre d'un treillis distributif $\mathbf{T}$ est l'ensemble Spec $\mathbf{T}$ de ses idéaux premiers, muni de la topologie suivante : une base d'ouverts est donnée par les

$$
\mathrm{D}_{\mathbf{T}}(a) \stackrel{\text { def }}{=}\{\mathfrak{p} \in \operatorname{Spec} \mathbf{T} \mid a \notin \mathfrak{p}\}, \quad a \in \mathbf{T} .
$$

On vérifie que

$$
\left.\begin{array}{ll}
\mathrm{D}_{\mathbf{T}}(a \wedge b)=\mathrm{D}_{\mathbf{T}}(a) \cap \mathrm{D}_{\mathbf{T}}(b), & \mathrm{D}_{\mathbf{T}}(0)=\emptyset, \\
\mathrm{D}_{\mathbf{T}}(a \vee b)=\mathrm{D}_{\mathbf{T}}(a) \cup \mathrm{D}_{\mathbf{T}}(b), & \mathrm{D}_{\mathbf{T}}(1)=\operatorname{Spec} \mathbf{T} .
\end{array}\right\}
$$

Le complémentaire de $\mathrm{D}_{\mathbf{T}}(a)$ est un fermé qu'on note $\mathrm{V}_{\mathbf{T}}(a)$.

On étend la notation $\mathrm{V}_{\mathbf{T}}(a)$ comme suit : si $I \subseteq \mathbf{T}$, on pose $\mathrm{V}_{\mathbf{T}}(I) \stackrel{\text { def }}{=} \bigcap_{x \in I} \mathrm{~V}_{\mathbf{T}}(x)$. Si $\mathcal{I}_{\mathbf{T}}(I)=\mathfrak{I}$, on a $\mathrm{V}_{\mathbf{T}}(I)=\mathrm{V}_{\mathbf{T}}(\mathfrak{I})$. On dit parfois que $\mathrm{V}_{\mathbf{T}}(\mathfrak{I})$ est la variété associée à $\mathfrak{I}$.

Les espaces topologiques homéomorphes à des espaces Spec $\mathbf{T}$ ont été appelés espaces spectraux par Stone [22].

Avec la logique classique et l'axiome du choix, l'espace Spec T a « suffisamment de points » : on peut retrouver le treillis $\mathbf{T}$ à partir de son spectre. Voici comment.

Tout d'abord on a le

\section{Théorème de Krull}

Supposons que $\mathfrak{J}$ est un idéal, $\mathfrak{F}$ un filtre et $\mathfrak{J} \cap \mathfrak{F}=\emptyset$. Alors il existe un idéal premier $\mathfrak{P}$ tel que $\mathfrak{J} \subseteq \mathfrak{P}$ et $\mathfrak{P} \cap \mathfrak{F}=\emptyset$.

On en déduit que :

- L'application $a \in \mathbf{T} \mapsto \mathrm{D}_{\mathbf{T}}(a) \in \mathcal{P}(\operatorname{Spec} \mathbf{T})$ est injective : elle identifie $\mathbf{T}$ à un treillis d'ensembles (théorème de représentation de Birkhoff).

- Si $\varphi: \mathbf{T} \rightarrow \mathbf{T}^{\prime}$ est un homomorphisme injectif l'application $\varphi^{\star}:$ Spec $\mathbf{T}^{\prime} \rightarrow$ Spec $\mathbf{T}$ obtenue par dualité est surjective.

- Tout idéal de $\mathbf{T}$ est intersection des idéaux premiers qui le contiennent.

- L'application $\mathfrak{I} \mapsto V_{\mathbf{T}}(\mathfrak{I})$, des idéaux de $\mathbf{T}$ vers les fermés de Spec $\mathbf{T}$ est un isomorphisme d'ensembles ordonnés (pour l'inclusion et l'inclusion renversée).

On montre aussi que les ouverts quasi-compacts de Spec $\mathbf{T}$ sont exactement les $\mathrm{D}_{\mathbf{T}}(a)$. D'après les égalités (17) les ouverts quasi-compacts de Spec $\mathbf{T}$ forment un treillis distributif de parties de Spec $\mathbf{T}$, isomorphe à $\mathbf{T}$.

A partir d'un espace spectral $X$ on peut considérer le treillis distributif $\operatorname{Oqc}(X)$ formé par ses ouverts quasi-compacts. Puisque pour tout treillis distributif $\mathbf{T}$, le treilli $\operatorname{Oqc}(\operatorname{Spec}(\mathbf{T}))$ est canoniquement isomorphe à $\mathbf{T}$, pour tout espace spectral $X, \operatorname{Spec}(\operatorname{Oqc}(X))$ est canoniquement homéomorphe à $X$.

On dit qu'un point $\mathfrak{q} \in X$ d'un espace spectral est le point générique du fermé $F$ si $F=$ $\overline{\{\mathfrak{q}\}}$. Ce point (s'il existe) est nécessairement unique car les espaces spectraux sont des espaces de Kolmogoroff (autrement dit, ils vérifient la condition de séparation $\mathrm{T}_{0}$ ). Les fermés $\overline{\{\mathfrak{q}\}}$ sont exactement tous les fermés irréductibles de $X$. La relation d'ordre $\mathfrak{q} \in \overline{\{\mathfrak{p}\}}$ sera notée $\mathfrak{p} \leq_{X} \mathfrak{q}$, et on a $\mathfrak{p} \leq_{X} \mathfrak{q} \Leftrightarrow \overline{\{\mathfrak{q}\}} \subseteq \overline{\{\mathfrak{p}\}}$. Les points fermés de Spec $\mathbf{T}$ sont les idéaux maximaux de $\mathbf{T}$.

Si $X=$ Spec $\mathbf{T}$ la relation $\mathfrak{p} \leq_{X} \mathfrak{q}$ est simplement la relation d'inclusion usuelle entre idéaux premiers.

Tout homomorphisme $\varphi: \mathbf{T} \rightarrow \mathbf{T}^{\prime}$ de treillis distributifs fournit par dualité une application continue $\varphi^{\star}$ : Spec $\mathbf{T}^{\prime} \rightarrow$ Spec $\mathbf{T}$, qui est appelée une application spectrale. Pour qu'une application continue entre espaces spectraux soit spectrale il faut et il suffit que l'image réciproque de tout ouvert quasi-compact soit un ouvert quasi-compact. 
La catégorie spectrale ainsi définie est alors équivalente à la catégorie opposée à celle des treillis distributifs.

Remarque. - Une définition purement topologique des espaces spectraux est la suivante [22] :

- L'espace est de Kolmogoroff (i.e., de type $\mathrm{T}_{0}$ ) : étant donnés deux points il existe un voisinage de l'un des deux qui ne contient pas l'autre.

- L'espace est quasi-compact.

- L'intersection de deux ouverts quasi-compacts est un ouvert quasi-compact.

- Tout ouvert est réunion d'ouverts quasi-compacts.

- Pour tout fermé $F$ et pour tout ensemble $S$ d'ouverts quasi-compacts tels que $F \cap \bigcap_{U \in S^{\prime}} U \neq \emptyset$ pour toute partie finie $S^{\prime}$ de $S$, on a aussi $F \cap \bigcap_{U \in S} U \neq \emptyset$.

En présence des quatre premières propriétés la dernière peut se reformuler comme suit : tout fermé irréductible admet un point générique.

On appelle espace de Stone un espace spectral Spec $\mathbf{T}$ lorsque $\mathbf{T}$ est une algèbre de Boole. Il est bien connu que les espaces de Stone peuvent être caractérisés comme les espaces compacts totalement discontinus.

En mathématiques constructives. - D'un point de vue constructif, T est une version « sans points » de Spec $\mathbf{T}$. En d'autres termes, à défaut d'avoir accès aux points de Spec $\mathbf{T}$, on peut se contenter de l'ensemble de ses ouverts quasi-compacts, qui sont directement visibles (sans recours à l'axiome du choix ni au principe du tiers exclu). La version sans points est plus facile à appréhender. Au contraire les points de Spec $\mathbf{T}$ ne sont pas en général des objets accessibles sans recours à des principes non constructifs.

En mathématiques constructives on a a priori plusieurs possibilités pour définir le spectre d'un treillis distributif (toutes équivalentes en mathématiques classiques). Le plus raisonnable semble de définir Spec $\mathbf{T}$ comme l'ensemble des filtres premiers de $\mathbf{T}$, c'est-à-dire les filtres pour lesquels on a

$$
x \wedge y \in \mathfrak{F} \quad \Longrightarrow \quad x \in \mathfrak{F} \text { ou } y \in \mathfrak{F}
$$

avec un $«$ ou $»$ explicite. Mais de tels espaces Spec T n'ont pas toujours suffisamment de points ${ }^{(1)}$ et on ne peut pas affirmer constructivement que les deux catégories sont antiéquivalentes, du moins si on définit les morphismes entre espaces spectraux comme des applications, car les applications nécessitent des points.

Une solution alternative satisfaisante (mais un peu troublante au premier abord) est de considérer Spec $\mathbf{T}$ comme un « espace topologique sans points », c'est-à-dire un espace topologique défini uniquement à travers sa base d'ouverts $\mathrm{D}_{\mathbf{T}}(a)$ (où $a$ parcourt $\mathbf{T}$ ). Les morphismes sont alors définis de manière purement formelle comme donnés par les morphismes des treillis correspondants, en renversant le sens des flèches. De ce point de vue l'antiéquivalence de la catégorie spectrale et de la catégorie des treillis distributifs devient une pure tautologie définitionnelle.

En tout état de cause, bien que la catégorie spectrale reste utile pour l'intuition, tout le travail se fait dans la catégorie des treillis distributifs. L'avantage est naturellement que l'on obtient des théorèmes constructifs.

Dans cet article les spectres seront étudiés uniquement du point de vue des mathématiques classiques, comme source d'inspiration importante pour de bonnes notions concernant les treillis distributifs.

Espaces spectraux nœthériens. - Un espace spectral $X$ est dit nœthérien si toute suite croissante d'ouverts est stationnaire. Il revient au même de dire que tout ouvert est quasi-compact, ou encore que le treillis $\operatorname{Oqc}(X)$ est nœthérien. Dans un espace spectral nœthérien tout ouvert est un $\mathrm{D}_{\mathbf{T}}(a)$ et tout fermé un $\mathrm{V}_{\mathbf{T}}(b)$.

\footnotetext{
${ }^{(1)}$ On peut par exemple définir un treillis distributif infini dénombrable explicite qui ne possède pas d'idéaux premiers récursifs. Pour un tel treillis distributif, il ne peut pas y avoir de preuve constructive que Spec $\mathbf{T}$ est non vide
} 
Deux autres topologies intéressantes sur Spec T. - En mathématiques classiques on a une bijection canonique entre les ensembles sous-jacents aux espaces $\operatorname{Spec} \mathbf{T}$ et $\operatorname{Spec} \mathbf{T}^{\circ}$ (à un idéal premier on associe son complémentaire) qui permet d'identifier ces deux ensembles. La topologie n'est pas la même. Les ouverts de base de Spec $\mathbf{T}^{\circ}$ sont les $\mathrm{V}_{\mathbf{T}}(a)$. Si $X=\operatorname{Spec} \mathbf{T}$ et $X^{\prime}=\operatorname{Spec} \mathbf{T}^{\circ}$, et si on identifie les ensembles sous-jacents à $X$ et $X^{\prime}$, la relation d'ordre $\leq_{X^{\prime}}$ est la relation symétrique de $\leq_{X}$ (l'ordre est renversé), mais ce qui se passe pour la topologie est plus compliqué.

On doit également considérer la topologie constructible (patch topology) dont les ouverts de base sont les $\mathrm{D}_{\mathbf{T}}(a) \cap \mathrm{V}_{\mathbf{T}}(b)$. Cela donne un espace compact naturellement homémorphe à Spec $\mathbf{T}^{\text {bool }}$ où $\mathbf{T}^{\text {bool }}$ est le treillis booléen engendré par $\mathbf{T}$. En mathématiques classiques on obtient $\mathbf{T}^{\text {bool }}$ comme la sous algèbre de Boole de l'ensemble des parties de Spec $\mathbf{T}$ engendrée par les $\mathrm{D}_{\mathbf{T}}(a)$. Ce treillis peut aussi être décrit constructivement comme suit (cf. [2]). On considère une copie disjointe de $\mathbf{T}$, qu'on note $\dot{\mathbf{T}}$. Alors $\mathbf{T}^{\text {bool }}$ est un treillis distributif défini par générateurs et relations. Les générateurs sont les éléments de l'ensemble $T_{1}=\mathbf{T} \cup \mathbf{T}$ et les relations sont obtenues comme suit : si $A, F, B, E$ sont quatre parties finies de $\mathbf{T}$ on a

$$
\bigwedge A \wedge \bigwedge E \leq_{\mathbf{T}} \bigvee B \vee \bigvee F \Longrightarrow \bigwedge A \wedge \bigwedge \dot{F} \leq_{T_{1}} \bigvee B \vee \bigvee \dot{E}
$$

On montre que $\mathbf{T}$ et $\dot{\mathbf{T}}$ s'injectent naturellement dans $\mathbf{T}^{\text {bool }}$ et que l'implication ci-dessus est en fait une équivalence. On obtient par dualité deux applications spectrales bijectives Spec $\mathbf{T}^{\text {bool }} \rightarrow$ Spec $\mathbf{T}$ et Spec $\mathbf{T}^{\text {bool }} \rightarrow \operatorname{Spec} \mathbf{T}^{\circ}$.

Espaces spectraux finis. - Dans le cas des treillis distributifs finis on obtient les espaces spectraux finis, qui ne sont rien d'autre que les ensembles ordonnés finis, (car il suffit de connaitre l'adhérence des points pour connaître la topologie) avec pour base d'ouverts les $\downarrow a$. Les ouverts sont tous quasi-compacts, ce sont les parties initiales, et les fermés sont les parties finales. Enfin, une application entre espaces spectraux finis est spectrale si et seulement si elle est croissante (pour les relations d'ordres associées).

La notion d'espace spectral apparaît ainsi comme une généralisation pertinente de la notion d'ensemble ordonné fini au cas infini.

$\mathrm{Si}$ on identifie les ensembles sous-jacents à $\operatorname{Spec} \mathbf{T}$ et $\operatorname{Spec} \mathbf{T}^{\circ}$ les deux spectres sont presque les mêmes : c'est le même ensemble ordonné au renversement près de la relation d'ordre. En outre les ouverts et les fermés sont échangés.

2.2. Treillis quotients et sous espaces spectraux. - En utilisant l'antiéquivalence des catégories, on définit la notion de sous espace spectral comme duale de la notion de treillis quotient. Ce sera l'objet du théorème 2.2.

Caractérisation des sous espaces spectraux. - Nous commençons par un lemme facile, qui caractérise les points de Spec $\mathbf{T}$ qui $«$ sont des éléments de $\operatorname{Spec} \mathbf{T}^{\prime} »$ lorsque $\mathbf{T}^{\prime}$ est un quotient de $\mathbf{T}$.

Lemme 2.1. - Soit $\mathbf{T}^{\prime}$ un treillis quotient de $\mathbf{T}$ et $\pi: \mathbf{T} \rightarrow \mathbf{T}^{\prime}$ la projection canonique. Notons $X=\operatorname{Spec} \mathbf{T}^{\prime}, Y=\operatorname{Spec} \mathbf{T}$ et $\pi^{\star}: X \rightarrow Y$ l'injection duale de $\pi$. Rappelons que pour un idéal premier $\mathfrak{p}$ de $\mathbf{T}$ nous notons $\theta_{\mathfrak{p}}: \mathbf{T} \rightarrow \mathbf{2}$ l'homomorphisme correspondant de noyau $\mathfrak{p}$. Les propriétés suivantes sont équivalentes:

$-\mathfrak{p} \in \pi^{\star}\left(\operatorname{Spec} \mathbf{T}^{\prime}\right)$.

$-\theta_{\mathfrak{p}}$ se factorise par $\mathbf{T}^{\prime}$.

$-\forall a, b \in \mathbf{T}((a \preceq b, b \in \mathfrak{p}) \Rightarrow a \in \mathfrak{p})$.

Cela peut se reformuler comme suit. Si le treillis quotient $\mathbf{T}^{\prime}$ est défini par un système $R$ de relations $x_{i}=y_{i}$, les propriétés suivantes sont équivalentes :

$-\mathfrak{p} \in \pi^{\star}\left(\operatorname{Spec} \mathbf{T}^{\prime}\right)$.

- $\theta_{\mathfrak{p}}$ 《 réalise un modèle de $R »$, c'est-à-dire $\forall i \theta_{\mathfrak{p}}\left(x_{i}\right)=\theta_{\mathfrak{p}}\left(y_{i}\right)$.

$-\forall i\left(x_{i} \in \mathfrak{p} \Leftrightarrow y_{i} \in \mathfrak{p}\right)$. 
Dans le théorème suivant nous identifions $\operatorname{Spec}^{\prime} \mathbf{T}^{\prime}$ à une partie de Spec $\mathbf{T}$ au moyen de l'injection $\pi^{\star}$

Théorème 2.2. - (caractérisations des sous espaces spectraux)

1. Avec les notations du lemme 2.1, $X$ est un sous espace topologique de $Y$. En outre $\operatorname{Oqc}(X)=$ $\{U \cap X \mid U \in \operatorname{Oqc}(Y)\}$. On dit que $X$ est un sous espace spectral de $Y$.

2. Pour qu'une partie $X$ d'un espace spectral $Y$ soit un sous espace spectral il faut et suffit que les conditions suivantes soient vérifiées :

- La topologie induite par $Y$ fait de $X$ un espace spectral, et

- $\operatorname{Oqc}(X)=\{U \cap X \mid U \in \operatorname{Oqc}(Y)\}$.

3. Une partie $X$ d'un espace spectral $Y$ est un sous espace spectral si et seulement si elle est fermée pour la topologie constructible.

4. Si $Z$ est une partie arbitraire d'un espace spectral $Y=$ Spec $\mathbf{T}$ son adhérence pour la topologie constructible est égal à $X=\mathrm{Spec}^{\mathbf{T}}$ où $\mathbf{T}^{\prime}$ est le treillis quotient de $\mathbf{T}$ défini par la relation de préordre $\preceq$ suivante :

$$
a \preceq b \quad \Longleftrightarrow \quad\left(\mathrm{D}_{\mathbf{T}}(a) \cap Z\right) \subseteq\left(\mathrm{D}_{\mathbf{T}}(b) \cap Z\right)
$$

En outre, $X$ est le plus petit sous espace spectral de $Y$ contenant $Z$.

Démonstration. - Le point 1 est facile, et définit la notion de sous espace spectral. Le point 2 en résulte. Le point 3 résulte des points 2 et 4 . Montrons le point 4 .

Remarquons tout d'abord que la relation (18) définit bien un treillis quotient $\mathbf{T}^{\prime}$ car les relations (11) sont trivialement vérifiées si on tient compte des relations (17).

Montrons que $X=\operatorname{Spec} \mathbf{T}^{\prime}$ est le plus petit sous espace spectral de $Y$ contenant $Z$.

Tout d'abord $Z \subseteq X$ : soit $\mathfrak{p} \in Z$, nous voulons montrer que si $b \in \mathfrak{p}$ et $a \preceq b$ alors $a \in \mathfrak{p}$. Si $\mathrm{D}_{\mathbf{T}}(a) \cap Z \subseteq \mathrm{D}_{\mathbf{T}}(b)$ et $b \in \mathfrak{p}$ alors $\mathfrak{p} \notin \mathrm{D}_{\mathbf{T}}(b)$ donc $\mathfrak{p} \notin \mathrm{D}_{\mathbf{T}}(a) \cap Z$ donc $\mathfrak{p} \notin \mathrm{D}_{\mathbf{T}}(a)$ c'est-à-dire $a \in \mathfrak{p}$. Par ailleurs $X$ est minimal. En effet effet si $X_{1}=$ Spec $\mathbf{T}_{1}$ est un sous espace spectral de $Y$ contenant $Z$, on a $a \leq \mathbf{T}_{1} b \Leftrightarrow\left(\mathrm{D}_{\mathbf{T}}(a) \cap X_{1}\right) \subseteq\left(\mathrm{D}_{\mathbf{T}}(b) \cap X_{1}\right)$ ce qui implique $\left(\mathrm{D}_{\mathbf{T}}(a) \cap Z\right) \subseteq\left(\mathrm{D}_{\mathbf{T}}(b) \cap Z\right)$ et donc $a \leq \mathbf{T}^{\prime} b$, d'où $X \subseteq X_{1}$.

Il reste à montrer que $X$ est l'adhérence de $Z$ pour la topologie constructible. Notons $\widetilde{Z}$ cette adhérence. Nous voulons donc démontrer pour tout $\mathfrak{p} \in$ Spec T l'équivalence des deux propriétés suivantes :

1. $\mathfrak{p} \in \widetilde{Z}$, c'est-à-dire :

$$
\forall a, b \in \mathbf{T},\left(\mathfrak{p} \in \mathrm{D}_{\mathbf{T}}(a) \cap \mathrm{V}_{\mathbf{T}}(b) \Longrightarrow \mathrm{D}_{\mathbf{T}}(a) \cap \mathrm{V}_{\mathbf{T}}(b) \cap Z \neq \emptyset\right),
$$

2. $\mathfrak{p} \in \operatorname{Spec} \mathbf{T}^{\prime}$.

Or le point 2. équivaut successivement à

3. $\forall a, b \in \mathbf{T},((a \preceq b, b \in \mathfrak{p}) \Rightarrow a \in \mathfrak{p})$.

4. $\forall a, b \in \mathbf{T},(a \preceq b, b \in \mathfrak{p}$ et $a \notin \mathfrak{p})$ sont incompatibles.

5. $\forall a, b \in \mathbf{T}, \mathrm{D}_{\mathbf{T}}(a) \cap Z \subseteq \mathrm{D}_{\mathbf{T}}(b)$ et $\mathfrak{p} \in \mathrm{D}_{\mathbf{T}}(a) \cap \mathrm{V}_{\mathbf{T}}(b)$ sont incompatibles.

6. $\forall a, b \in \mathbf{T}, \mathrm{D}_{\mathbf{T}}(a) \cap \mathrm{V}_{\mathbf{T}}(b) \cap Z=\emptyset$ et $\mathfrak{p} \in \mathrm{D}_{\mathbf{T}}(a) \cap \mathrm{V}_{\mathbf{T}}(b)$ sont incompatibles.

et le dernier point est clairement équivalent à 1 .

Corollaire 2.3. - Toute réunion finie et toute intersection de sous espaces spectraux de $X=$ Spec $\mathbf{T}$ est un sous espace spectral. Si $X_{i}=\operatorname{Spec}_{i}$ pour un quotient $\pi_{i}: \mathbf{T} \rightarrow \mathbf{T}_{i}$ alors $\bigcap_{i} X_{i}$ correspond au quotient engendré par toutes les relations $\pi_{i}(x)=\pi_{i}(y)$. Si la famille est finie $\bigcup_{i} X_{i}$ correspond au quotient par la relation $\&_{i}\left(\pi_{i}(x)=\pi_{i}(y)\right)$.

Proposition 2.4. - (Ouverts et fermés de base)

1. $\mathrm{D}_{\mathbf{T}}(a)$ est un sous espace spectral de $X$ canoniquement homéomorphe à $\operatorname{Spec}(\mathbf{T} /(a=1))$. 
2. $\mathrm{V}_{\mathbf{T}}(b)$ est un sous espace spectral de $X$ canoniquement homéomorphe à $\operatorname{Spec}(\mathbf{T} /(b=0))$.

Démonstration. — Soit $x \preceq y$ l'ordre partiel correspondant au sous espace spectral $\mathrm{D}_{\mathbf{T}}(a)$. On a donc :

$$
\begin{aligned}
x \preceq y & \Longleftrightarrow \mathrm{D}_{\mathbf{T}}(x) \cap \mathrm{D}_{\mathbf{T}}(a) \subseteq \mathrm{D}_{\mathbf{T}}(y) \cap \mathrm{D}_{\mathbf{T}}(a) \\
& \Longleftrightarrow \mathrm{D}_{\mathbf{T}}(x \wedge a) \subseteq \mathrm{D}_{\mathbf{T}}(y \wedge a) \\
& \Longleftrightarrow x \wedge a \leq y \wedge a
\end{aligned}
$$

et ceci est bien le préordre correspondant au quotient $\operatorname{Spec}(\mathbf{T} /(a=1))$.

Soit maintenant $x \preceq^{\prime} y$ l'ordre partiel correspondant au sous espace spectral $\mathrm{V}_{\mathbf{T}}(b)$. On a :

$$
\begin{aligned}
x \preceq^{\prime} y & \Longleftrightarrow \mathrm{D}_{\mathbf{T}}(x) \cap \mathrm{V}_{\mathbf{T}}(b) \subseteq \mathrm{D}_{\mathbf{T}}(y) \cap \mathrm{V}_{\mathbf{T}}(b) \\
& \Longleftrightarrow \mathrm{D}_{\mathbf{T}}(x) \cup \mathrm{D}_{\mathbf{T}}(b) \subseteq \mathrm{D}_{\mathbf{T}}(y) \cup \mathrm{D}_{\mathbf{T}}(b) \\
& \Longleftrightarrow \mathrm{D}_{\mathbf{T}}(x \vee b) \subseteq \mathrm{D}_{\mathbf{T}}(y \vee b) \\
& \Longleftrightarrow x \vee b \leq y \vee b
\end{aligned}
$$

et ceci est bien le préordre correspondant au quotient $\operatorname{Spec}(\mathbf{T} /(b=0))$.

Fermés de Spec T. — Dans ce paragraphe $\mathbf{T}$ est un treillis distributif fixé et $X=$ Spec T. Si $Z \subseteq X$ on notera $\bar{Z}$ l'ahérence de $Z$ pour la topologie usuelle de $X$.

Proposition 2.5. - (Fermés de Spec T)

1. Un fermé arbitraire de Spec $\mathbf{T}$ est de la forme $\mathrm{V}_{\mathbf{T}}(\mathfrak{J})=\bigcap_{x \in \mathfrak{J}} \mathrm{V}_{\mathbf{T}}(x)$ où $\mathfrak{J}$ est un idéal arbitraire de $\mathbf{T}$. C'est un sous espace spectral et il correspond au quotient $\mathbf{T} /(\mathfrak{J}=0)$.

2. L'intersection d'une famille de fermés correspond au sup de la famille d'idéaux. La réunion de deux fermés correspond à l'intersection des deux idéaux.

3. Le treillis $\mathbf{T} /((a: b)=0)$ est le quotient correspondant $\grave{a} \overline{\mathrm{V}_{\mathbf{T}}(a) \cap \mathrm{D}_{\mathbf{T}}(b)}$.

4. Donc $\mathbf{T}$ est une algèbre de Heyting si et seulement si $X$ vérifie la propriété suivante : pour tous ouverts quasi-compacts $U_{1}$ et $U_{2}$, l'adhérence de $U_{1} \backslash U_{2}$ est un ouvert quasi-compact.

5. À l'adhérence de $\mathrm{D}_{\mathbf{T}}(x)$ correspond le quotient $\mathbf{T} /((0: x)=0)$.

6. Donc à la frontière de $\mathrm{D}_{\mathbf{T}}(x)$ correspond le quotient $\mathbf{T}_{\mathrm{K}}^{x}=\mathbf{T} /\left(\mathrm{K}_{\mathbf{T}}^{x}=0\right)$, où

$$
\mathrm{K}_{\mathbf{T}}^{x}=\downarrow x \vee(0: x)
$$

Le treillis $\mathbf{T}_{\mathrm{K}}^{x}$ sera appelé le bord supérieur (de Krull) de $x$ dans $\mathbf{T}$. On dira aussi que $\mathrm{K}_{\mathbf{T}}^{x}$ est l'idéal bord de Krull de $x$ dans $\mathbf{T}$.

Lorsque $\mathbf{T}$ est une algèbre de Heyting, $\mathrm{K}_{\mathbf{T}}^{x}=\downarrow(x \vee \neg x)$ et $\mathbf{T}_{\mathrm{K}}^{x} \simeq \uparrow(x \vee \neg x)$ avec l'homomorphisme surjectif $\pi_{\mathrm{K}}^{x}: \mid \begin{aligned} \mathbf{T} & \rightarrow \uparrow(x \vee \neg x) \\ y & \mapsto y \vee x \vee \neg x\end{aligned}$.

Démonstration. - Pour le seul point délicat (le point 3), on dit : puisque $(a: b)=\{x \mid x \wedge b \leq a\}$, la variété associée $\mathrm{V}_{\mathbf{T}}(a: b)$ est l'intersection des $\mathrm{V}_{\mathbf{T}}(x)$ tels que $\mathrm{V}_{\mathbf{T}}(a) \subseteq \mathrm{V}_{\mathbf{T}}(x) \cup \mathrm{V}_{\mathbf{T}}(b)$, c'està-dire encore tels que $\mathrm{V}_{\mathbf{T}}(a) \cap \mathrm{D}_{\mathbf{T}}(b) \subseteq \mathrm{V}_{\mathbf{T}}(x)$. Or tout fermé de Spec $\mathbf{T}$ est une intersection de fermés de base $\mathrm{V}_{\mathbf{T}}(x)$, donc on obtient bien l'adhérence de $\mathrm{V}_{\mathbf{T}}(a) \cap \mathrm{D}_{\mathbf{T}}(b)$.

Remarques. -

1) On notera qu'un ouvert arbitraire de $X$ n'est pas en général un sous espace spectral.

2) La définition que nous avons donnée pour le treillis bord $\mathbf{T}_{\mathrm{K}}^{x}$ est constructive, (elle ne nécessite pas que l'espace Spec T ait suffisamment de points) mais elle est motivée par l'interprétation qu'on donne de son spectre en mathématiques classiques.

Signalons le lemme suivant qui permet de mieux cerner l'idéal bord de Krull de $x$ dans $\mathbf{T}$.

Lemme 2.6. - Pour tout $x \in \mathbf{T}$ l'idéal $\mathbf{j}$ bord de Krull de $x$ dans $\mathbf{T}$ est régulier, c'est-à-dire «son annulateur est réduit à $0 »: 0: \mathbf{j}=0$. 
Démonstration. - Soit $u \in(0: \mathfrak{j})$. Puisque $\mathfrak{j}=\downarrow x \vee(0: x)$ on a $u \wedge x=0$ et, pour tout $z \in(0: x)$, $u \wedge z=0$. En particulier $u \wedge u=0$.

Notez que dans le cas d'une algèbre de Heyting il ne s'agit de rien d'autre que de la loi découverte par Brouwer : $\neg(x \vee \neg x)=0$.

La proposition qui suit est la version duale, constructive, «sans points » du fait topologique suivant : si $A$ et $B$ sont fermés, la réunion des bords de $A \cup B$ et de $A \cap B$ est égale à celle des bords de $A$ et $B$.

Proposition 2.7. - Pour tous $x, y \in \mathbf{T}$ on $a$ :

$$
\mathrm{K}_{\mathbf{T}}^{x} \cap \mathrm{K}_{\mathbf{T}}^{y}=\mathrm{K}_{\mathbf{T}}^{x \vee y} \cap \mathrm{K}_{\mathbf{T}}^{x \wedge y} .
$$

Démonstration. - Soit $z \in \mathrm{K}_{\mathbf{T}}^{x} \cap \mathrm{K}_{\mathbf{T}}^{y}$, autrement dit il existe $u$ et $v$ tels que $z \leq x \vee u$ et $u \wedge x=0$, $z \leq y \vee v$ et $v \wedge y=0$. Alors $z \leq(x \vee(u \vee v)) \wedge(y \vee(u \vee v))=(x \wedge y) \vee(u \vee v)$ avec $(u \vee v) \wedge(x \wedge y)=$ $(u \wedge(x \wedge y)) \vee(v \wedge(x \wedge y))=0 \vee 0=0$ donc $z \in \mathrm{K}_{\mathbf{T}}^{x \wedge y}$

De même $z \leq(x \vee y) \vee(u \wedge v)$ avec $(u \wedge v) \wedge(x \vee y)=0$ donc $z \in \mathrm{K}_{\mathbf{T}}^{x \vee y}$.

Enfin supposons $z \in \mathrm{K}_{\mathbf{T}}^{x \vee y} \cap \mathrm{K}_{\mathbf{T}}^{x \wedge y}$, autrement dit il existe $u$ et $v$ tels que $z \leq x \vee y \vee u$ et $u \wedge(x \vee y)=0$, $z \leq(x \wedge y) \vee v$ et $v \wedge x \wedge y=0$. Soit $u_{1}=(y \vee u) \wedge v$. On a $z \leq(x \wedge y) \vee v \leq x \vee v$ et $z \leq x \vee(y \vee u)$ donc $z \leq x \vee u_{1}$. Par ailleurs $x \wedge u_{1}=x \wedge(y \vee u) \wedge v \leq x \wedge y \wedge v=0$ et donc $z \in \mathrm{K}_{\mathbf{T}}^{x}$.

Fermés de Spec $\mathbf{T}^{\circ}$. — Rappelons que la notion opposée à l'idéal $a: b$ est le filtre $a \backslash b \stackrel{\text { def }}{=}$ $\{z \mid z \vee b \geq a\}$.

Introduisons aussi la notation suivante, pour $F \subseteq \mathbf{T}: \mathrm{D}_{\mathbf{T}}(F)=\bigcap_{a \in \mathfrak{F}} \mathrm{D}_{\mathbf{T}}(a)$. Si $\mathfrak{F}$ est le filtre engendré par $F$, on a $\mathrm{D}_{\mathbf{T}}(F)=\mathrm{D}_{\mathbf{T}}(\mathfrak{F})$.

La proposition suivante découle de la proposition 2.5 par renversement de l'ordre (on n'a réécrit que les points 1 et 6 ) modulo l'identification des ensembles sous-jacents à Spec $\mathbf{T}$ et Spec $^{\circ}$.

Proposition 2.8. - (Fermés de Spec $\mathbf{T}^{\circ}$ )

1. Si $\mathfrak{F}$ est un filtre arbitraire, le quotient $\mathbf{T} /(\mathfrak{F}=1)$ correspond au sous espace spectral intersection $\bigcap_{a \in \mathfrak{F}} \mathrm{D}_{\mathbf{T}}(a)$. C'est un fermé arbitraire pour la topologie de $\operatorname{Spec} \mathbf{T}^{\circ}$ (donc il est a fortiori fermé pour la topologie constructible).

2. On définit le quotient $\mathbf{T}_{x}^{\mathrm{K}}=\mathbf{T} /\left(\mathrm{K}_{x}^{\mathbf{T}}=1\right)$, où $\mathrm{K}_{x}^{\mathbf{T}}$ est le filtre

$$
\mathrm{K}_{x}^{\mathbf{T}}=\uparrow x \wedge(1 \backslash x)
$$

Le treillis $\mathbf{T}_{x}^{\mathrm{K}}$ sera appelé le bord inférieur (de Krull) de $x$ dans $\mathbf{T}$. On dira aussi que $\mathrm{K}_{x}^{\mathbf{T}}$ est le filtre bord de Krull de $x$.

Lorsque $\mathbf{T}$ est une algèbre de Brouwer, $\mathrm{K}_{x}^{\mathbf{T}}=\uparrow(x \wedge(1-x))$ et $\mathbf{T}_{x}^{\mathrm{K}} \simeq \downarrow(x \wedge(1-x))$ avec l'homomorphisme surjectif

$$
\pi_{x}^{\mathrm{K}}: \mid \begin{aligned}
\mathbf{T} & \rightarrow \downarrow(x \wedge(1-x)) \\
y & \mapsto y \wedge x \wedge(1-x)
\end{aligned} .
$$

Le quotient $\mathbf{T}_{x}^{\mathrm{K}}$ correspond à la notion opposée à la frontière (c'est la frontière de $\mathrm{D}_{\mathbf{T}}(x)$ pour la topologie de Spec $\mathbf{T}^{\circ}$ ).

Recollement d'espaces spectraux. - Voici ce que donnent par dualité la proposition 1.8 et le fait 1.7.

Proposition 2.9. - (Recollement d'espaces spectraux le long de sous espaces spectraux)

1. Soit $\left(X_{i}\right)_{1 \leq i \leq n}$ une famille finie d'espaces spectraux, et pour chaque $i \neq j$ un sous-espace spectral $X_{i j}$ de $X_{i}$ avec un isomorphisme $\varphi_{i j}: X_{i j} \rightarrow X_{j i}$. Si les relations de compatibilité naturelles sont vérifiées, la limite inductive du diagramme dans la catégorie des espaces spectraux est un espace $X$ pour lequel chacun des $X_{i}$ est un sous-espace spectral. 
2. Si chaque $X_{i j}$ est un fermé de $X_{i}$ alors les $X_{i}$ sont fermés dans $X$ et la limite inductive est aussi valide dans la catégorie des espaces topologiques.

3. Si chaque $X_{i j}$ est un ouvert quasi-compact de $X_{i}$ alors les $X_{i}$ sont ouverts quasi-compacts dans $X$ et la limite inductive est aussi valide dans la catégorie des espaces topologiques.

Notez qu'on ne dit pas dans le point 1 que $X$ est la limite inductive des $X_{i}$ dans la catégorie des espaces topologiques.

Spectre maximal et spectre de Heitmann. - Dans un article remarquable [15] R. Heitmann explique que la notion usuelle de j-spectrum pour un anneau commutatif n'est pas la bonne dans le cas non nœthérien car elle ne correspond pas à un espace spectral au sens de Stone. Il introduit la modification suivante de la définition usuelle : au lieu de considérer l'ensemble des idéaux premiers qui sont intersections d'idéaux maximaux il propose de considérer l'adhérence du spectre maximal dans le spectre premier, adhérence à prendre au sens de la topologie constructible (la «patch topology »).

Définitions 2.10. - Soit $\mathbf{T}$ un treillis distributif.

1. On note Max $\mathbf{T}$ le sous-espace topologique de Spec $\mathbf{T}$ formé par les idéaux maximaux de $\mathbf{T}$. On l'appelle le spectre maximal de $\mathbf{T}$.

2. On note jspec $\mathbf{T}$ le sous espace topologique de $\operatorname{Spec} \mathbf{T}$ formé par les $\mathfrak{p}$ qui vérifient $J_{\mathbf{T}}(\mathfrak{p})=\mathfrak{p}$, c'est-à-dire les idéaux premiers $\mathfrak{p}$ qui sont intersections d'idéaux maximaux (c'est le j-spectrum « usuel »).

3. On appelle J-spectre de Heitmann de $\mathbf{T}$ et on note Jspec $\mathbf{T}$ l'adhérence du spectre maximal dans Spec $\mathbf{T}$, adhérence à prendre au sens de la topologie constructible. Cet ensemble est muni de la topologie induite par Spec $\mathbf{T}$.

4. On note Min $\mathbf{T}$ le sous-espace topologique de Spec $\mathbf{T}$ formé par les idéaux premiers minimaux de $\mathbf{T}$. On l'appelle le spectre minimal de $\mathbf{T}$.

Notez que malgré leurs dénominations, Max $\mathbf{T}$, jspec $\mathbf{T}$ et $\operatorname{Min} \mathbf{T}$ ne sont pas en général des espaces spectraux.

Théorème 2.11. - Soit $\mathbf{T}$ un treillis distributif. L'espace Jspec $\mathbf{T}$ est un sous espace spectral de Spec $\mathbf{T}$ canoniquement homéomorphe à $\mathrm{Spec} \mathrm{He}(\mathbf{T})$. Plus précisément, si $M$ est l'ensemble des idéaux maximaux dans $\operatorname{Spec} \mathbf{T}$, pour $a, b \in \mathbf{T}$ on $a$ :

$$
\mathrm{D}_{\mathbf{T}}(a) \cap M \subseteq \mathrm{D}_{\mathbf{T}}(b) \cap M \quad \Longleftrightarrow \quad a \preceq \text { He(T) } b .
$$

Démonstration. - Le treillis $\mathrm{He}(\mathbf{T})$ a été défini page 62 .

La deuxième affirmation implique la première. En effet Jspec $\mathbf{T}$, d'après le théorème 2.2 (4), est le spectre du treillis quotient $\mathbf{T}^{\prime}$ correspondant à la relation de préordre $a \leq_{\mathbf{T}^{\prime}} b$ définie par $\mathrm{D}_{\mathbf{T}}(a) \cap M \subseteq \mathrm{D}_{\mathbf{T}}(b) \cap M$.

Pour le deuxième point on remarque que les propriétés suivantes sont équivalentes :

$$
\begin{aligned}
& \mathrm{D}_{\mathbf{T}}(a) \cap M \subseteq \mathrm{D}_{\mathbf{T}}(b) \cap M \\
& \mathrm{D}_{\mathbf{T}}(a) \cap \mathrm{V}_{\mathbf{T}}(b) \cap M=\emptyset \\
& \forall \mathfrak{m} \in M(b \notin \mathfrak{m} \text { ou } a \in \mathfrak{m}) \\
& \forall \mathfrak{m} \in M(b \in \mathfrak{m} \Rightarrow a \in \mathfrak{m})
\end{aligned}
$$

Et la dernière assertion revient à dire que, vu dans le treillis quotient $\mathbf{T} /(b=0), a$ appartient au radical de Jacobson. Cela signifie $a \in \mathrm{J}_{\mathbf{T}}(b)$, c'est-à-dire $a \preceq_{\mathrm{He}(\mathbf{T})} b$.

Quelques points de comparaison.

Fait 2.12. -

1. Spec $\mathbf{T}=$ Jspec $\mathbf{T}$ si et seulement si $\mathbf{T}=\mathrm{He}(\mathbf{T})$, c'est-à-dire si $\mathbf{T}$ est faiblement Jacobson. 
2. Max $\mathbf{T}=$ Jspec $\mathbf{T}$ si et seulement si $\mathrm{He}(\mathbf{T})$ est une algèbre de Boole.

3. Si $\mathbf{T}$ possède un complément de Brouwer, Max $\mathbf{T}$ est un fermé de Spec $\mathbf{T}$, correspondant $\grave{a}$ l'idéal $\mathrm{I}_{\max }(\mathbf{T})$. C'est un espace de Stone, il est égal à Jspec $\mathbf{T}$.

4. Min $\mathbf{T}=$ Jspec $\mathbf{T}^{\circ}$ si et seulement si $\mathrm{He}\left(\mathbf{T}^{\circ}\right)$ est une algèbre de Boole.

5. Si $\mathbf{T}$ possède une négation, Min $\mathbf{T}$ est un fermé de $\mathrm{Spec}_{\mathbf{T}}^{\circ}$, correspondant au filtre $\mathrm{F}_{\min }(\mathbf{T})$. C'est un espace de Stone, il est égal à Jspec $\mathbf{T}^{\circ}$.

Démonstration. - Le point 1 résulte du théorème 2.11. Pour le point 2, (on est en mathématiques classiques) on remarque qu'un treillis distributif est une algèbre de Boole si et seulement si ses idéaux premiers sont tous maximaux. Le point 3 résulte du point 2 et du fait 1.17. Les points 4 et 5 s'obtiennent à partir des points 2 et 3 en passant au treillis opposé.

\section{Dimensions de Krull et de Heitmann pour un treillis distributif}

Nous arrivons dans cette section au cour de l'article. Nous reprenons le point de vue des mathématiques constructives. Les seules preuves non constructives sont celles qui font le lien entre une notion classique et sa reformulation constructive.

3.1. Dimension de Krull des treillis distributifs. - En mathématiques classiques la dimension de Krull d'un treillis distributif est définie comme en algèbre commutative : c'est la borne supérieure des longueurs des chaînes strictement croissantes d'idéaux premiers.

Nous rappelons maintenant une version constructive élémentaire de la dimension de Krull $([\mathbf{4 ,} 6])$ en nous appuyant sur l'intuition suivante : une variété est de dimension $\leq k$ si et seulement si le bord de toute sous variété est de dimension $\leq k-1$.

Le théorème suivant en mathématiques classiques nous donne une bonne signification intuitive de la dimension de Krull dans un treillis distributif.

Théorème 3.1. - Soit un treillis distributif $\mathbf{T}$ engendré par une partie $S$ et $\ell$ un entier positif ou nul. Les conditions suivantes sont équivalentes.

1. Le treillis $\mathbf{T}$ est de dimension $\leq \ell$.

2. Pour tout $x \in S$ le bord $\mathbf{T}_{\mathrm{K}}^{x}$ est de dimension $\leq \ell-1$.

3. Pour tout $x \in S$ le bord $\mathbf{T}_{x}^{\mathrm{K}}$ est de dimension $\leq \ell-1$.

4. Pour tous $x_{0}, \ldots, x_{\ell} \in S$ il existe $a_{0}, \ldots, a_{\ell} \in \mathbf{T}$ vérifiant :

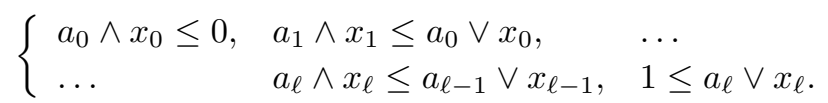

Si $\mathbf{T}$ est une algèbre de Heyting, les conditions précédentes sont aussi équivalentes à

5. Pour toute suite $x_{0}, \ldots, x_{\ell}$ dans $S$ on a l'égalité

$$
1=x_{\ell} \vee\left(x_{\ell} \rightarrow\left(\cdots\left(x_{1} \vee\left(x_{1} \rightarrow\left(x_{0} \vee \neg x_{0}\right)\right)\right) \cdots\right)\right)
$$

Si $\mathbf{T}$ est une algèbre de Brouwer, les conditions précédentes sont aussi équivalentes à

6. Pour toute suite $x_{0}, \ldots, x_{\ell}$ dans $S$ on a l'égalité

$$
0=x_{0} \wedge\left(x_{0}-\left(x_{1} \wedge\left(x_{1}-\left(\cdots\left(x_{\ell} \wedge\left(1-x_{\ell}\right)\right)\right)\right) \cdots\right)\right)
$$

En particulier un treillis est de dimension $\leq 0$ si et seulement si c'est une algèbre de Boole.

L'équivalence entre (1), (4) et (5) avait été établie précédemment dans [4], en poursuivant la problématique de Joyal [18] et Español [12].

Notez aussi que la théorie des algèbres de Heyting de dimension $\leq k$ est une théorie équationnelle. 
Démonstration. - Preuve du théorème 3.1 On commence par noter que le quotient $\mathbf{T}_{\mathrm{K}}^{x}=\mathbf{T} / \mathrm{K}_{\mathbf{T}}^{x}$ peut aussi être vu comme l'ensemble ordonné obtenu à partir de la relation de préordre $\leq^{x}$ définie sur $\mathbf{T}$ comme suit :

$$
a \leq^{x} b \quad \Longleftrightarrow \quad \exists y \in \mathbf{T}(x \wedge y=0 \quad \& a \leq x \vee y \vee b)
$$

$(1) \Leftrightarrow(2)$ : Nous montrons tout d'abord que tout filtre maximal $\mathfrak{f}$ de $\mathbf{T}$ devient trivial dans $\mathbf{T}_{\mathrm{K}}^{x}$, c'est-à-dire qu'il contient 0 . Autrement dit on doit trouver $a$ dans $\mathfrak{f}$ tel que $a \leq^{x} 0$. Si $x \in \mathfrak{f}$ alors on prend $a=x$ et $y=0$ dans (25). Si $x \notin \mathfrak{f}$ il existe $z \in \mathfrak{f}$ tel que $x \wedge z=0$ (puisque le filtre engendré par $\mathfrak{f}$ et $x$ est trivial dans $\mathbf{T}$ ) et on prend $a=y=z$ dans (25). Ceci montre que la dimension de $\mathbf{T}_{\mathrm{K}}^{x}$ chute de au moins une unité par rapport à celle de $\mathbf{T}$ (supposée finie).

Ensuite nous montrons que si on a deux filtres premiers $\mathfrak{f}^{\prime} \subseteq \mathfrak{f}, \mathfrak{f}$ maximal et $x \in \mathfrak{f} \backslash \mathfrak{f}^{\prime}$ alors $\mathfrak{f}^{\prime}$ ne devient pas trivial dans $\mathbf{T}_{\mathrm{K}}^{x}$ (ceci montre que la dimension de $\mathbf{T}_{\mathrm{K}}^{x}$ chute de seulement une unité si $x$ est bien choisi). En effet, dans le cas contraire, on aurait un $z \in \mathfrak{f}^{\prime}$ tel que $z \wedge x=0$, mais comme $z$ et $x \in \mathfrak{f}$ cela ferait $0 \in \mathfrak{f}$, ce qui est absurde.

Enfin nous remarquons que si $\mathfrak{f}^{\prime} \subseteq \mathfrak{f}$ sont des filtres premiers distincts et si $S$ engendre $\mathbf{T}$ on peut trouver $x \in S$ tel que $x \in \mathfrak{f} \backslash \mathfrak{f}^{\prime}$.

$(1) \Leftrightarrow(3)$ est conséquence de $(1) \Leftrightarrow(2)$ par renversement de l'ordre.

$(2) \Leftrightarrow(4)$ par récurrence sur $\ell$, vue la définition du bord.

$(2) \Leftrightarrow(5)$ par récurrence sur $\ell$, vue la définition du bord dans le cas d'une algèbre de Heyting.

$(3) \Leftrightarrow(6)$ par récurrence sur $\ell$, vue la définition du bord dans le cas d'une algèbre de Brouwer.

On a montré (en mathématiques classiques) que si $X$ est le spectre d'un treillis distributif $\mathbf{T}$ et $x \in \mathbf{T}$, alors la frontière de l'ouvert quasi-compact $\mathrm{D}_{\mathbf{T}}(x)$ de $X$ est (canoniquement isomorphe à) $\operatorname{Spec}\left(\mathbf{T}_{\mathrm{K}}^{x}\right)$. On obtient ainsi comme corollaire du théorème 3.1 le théorème suivant concernant la dimension des espaces spectraux (c'est-à-dire la longueur maximale des chaînes de fermés irréductibles, ou encore la longeur maximale des chaînes de points pour la relation $\mathfrak{p} \leq_{X} \mathfrak{q}$ ). Rappelons que l'unique espace spectral de dimension -1 est le vide.

Théorème 3.2. - Soit $k$ un entier $\geq 0$. Un espace spectral $X$ est de dimension $\leq k$ si et seulement si tout ouvert quasi-compact de $X$ a une frontière (un bord) de dimension $\leq k-1$.

Concernant la dimension de Krull, on choisit en mathématiques constructives la définition suivante :

Définition 3.3. - (définition constructive de la dimension de Krull)

La dimension de Krull (notée Kdim) des treillis distributifs est définie comme suit :

1. $\operatorname{Kdim}(\mathbf{T})=-1$ si et seulement si $1={ }_{\mathbf{T}} 0$ (i.e. le treillis est réduit à un point).

2. Pour $\ell \geq 0$ on définit $K \operatorname{dim}(\mathbf{T}) \leq \ell$ par les conditions équivalentes suivantes :

(a) $\forall x \in \mathbf{T}, \operatorname{Kdim}\left(\mathbf{T}_{\mathrm{K}}^{x}\right) \leq \ell-1$

(b) $\forall x \in \mathbf{T}, \operatorname{Kdim}\left(\mathbf{T}_{x}^{\mathrm{K}}\right) \leq \ell-1$

(c) $\forall x_{0}, \ldots, x_{\ell} \in \mathbf{T} \exists a_{0}, \ldots, a_{\ell} \in \mathbf{T}$ vérifiant :

$a_{0} \wedge x_{0} \leq 0, \quad a_{1} \wedge x_{1} \leq a_{0} \vee x_{0}, \ldots, \quad a_{\ell} \wedge x_{\ell} \leq a_{\ell-1} \vee x_{\ell-1}, \quad 1 \leq a_{\ell} \vee x_{\ell}$

Notez qu'il y a en fait trois définitions possibles ci-dessus pour $\operatorname{Kdim}(\mathbf{T}) \leq \ell$. Les définitions basées sur $2 \mathrm{a}$ et $2 \mathrm{~b}$ sont inductives, tandis que la définition basée sur 2c est globale. L'équivalence des définitions basées sur 2a et 2c est immédiate par induction (même chose pour 2b et 2c).

Remarque. - On peut illustrer le point 2.c dans la définition ci-dessus. Nous introduisons « l'idéal bord de Krull itéré ». Pour $x_{1}, \ldots, x_{n} \in \mathbf{T}$ nous notons $\mathbf{T}_{\mathrm{K}}\left[x_{1}\right]=\mathbf{T}_{\mathrm{K}}^{x_{1}}, \mathbf{T}_{\mathrm{K}}\left[x_{1}, x_{2}\right]=\left(\mathbf{T}_{\mathrm{K}}^{x_{1}}\right)_{\mathrm{K}}^{x_{2}}$, $\mathbf{T}_{\mathrm{K}}\left[x_{1}, x_{2}, x_{3}\right]=\left(\left(\mathbf{T}_{\mathrm{K}}^{x_{1}}\right)_{\mathrm{K}}^{x_{2}}\right)_{\mathrm{K}}^{x_{3}}$, etc. . les treillis bords quotients successifs, et $\mathrm{K}\left[\mathbf{T} ; x_{1}, \ldots, x_{k}\right]=$ $\mathrm{K}_{\mathbf{T}}\left[x_{1}, \ldots, x_{k}\right]$ désigne le noyau de la projection canonique $\mathbf{T} \rightarrow \mathbf{T}_{\mathrm{K}}\left[x_{1}, \ldots, x_{k}\right]$. Alors on a $y \in$ $\mathrm{K}_{\mathbf{T}}\left[x_{0}, \ldots, x_{\ell}\right]$ si et seulement si $\exists a_{0}, \ldots a_{\ell} \in \mathbf{T}$ vérifiant :

$$
a_{0} \wedge x_{0} \leq 0, \quad a_{1} \wedge x_{1} \leq a_{0} \vee x_{0}, \ldots, \quad a_{\ell} \wedge x_{\ell} \leq a_{\ell-1} \vee x_{\ell-1}, \quad y \leq a_{\ell} \vee x_{\ell}
$$


Si T est une algèbre de Heyting on a :

$$
\mathrm{K}_{\mathbf{T}}\left[x_{0}, \ldots, x_{\ell}\right]=\downarrow\left(x_{\ell} \vee\left(x_{\ell} \rightarrow\left(\cdots\left(x_{1} \vee\left(x_{1} \rightarrow\left(x_{0} \vee \neg x_{0}\right)\right)\right) \cdots\right)\right)\right)
$$

La dimension de Krull du treillis est $\leq \ell$ si et seulement si $1 \in \mathrm{K}_{\mathbf{T}}\left[x_{0}, \ldots, x_{\ell}\right]$ pour tous $x_{0}, \ldots, x_{\ell}$.

En mathématiques constructives la dimension de Krull de $\mathbf{T}$ n'est pas a priori un élément bien défini de $\mathbb{N} \cup\{-1\} \cup\{\infty\}$. En mathématiques classiques cet élément est défini comme la borne inférieure des entiers $\ell$ tels que $\operatorname{Kdim}(\mathbf{T}) \leq \ell$. On utilise en mathématiques constructives les notations suivantes $^{(2)}$, pour se rapprocher du langage classique :

Notation 3.4. - Soient $\mathbf{T}, \mathbf{L}, \mathbf{T}_{i}$ des treillis distributifs.

- $\mathrm{K} \operatorname{dim} \mathbf{L} \leq \mathrm{Kdim} \mathbf{T}$ signifie $: \forall \ell \geq-1(\mathrm{Kdim} \mathbf{T} \leq \ell \Rightarrow \mathrm{K} \operatorname{dim} \mathbf{L} \leq \ell)$.

- $\mathbf{K} \operatorname{dim} \mathbf{L}=\mathbf{K} \operatorname{dim} \mathbf{T}$ signifie $: \mathbf{K} \operatorname{dim} \mathbf{L} \leq \mathbf{K} \operatorname{dim} \mathbf{T}$ et $\mathbf{K} \operatorname{dim} \mathbf{L} \geq \mathbf{K} \operatorname{dim} \mathbf{T}$.

$-\mathrm{Kdim} \mathbf{T} \leq \sup _{i} \mathrm{Kdim} \mathbf{T}_{i}$ signifie :

$$
\forall \ell \geq-1\left(\&_{i}\left(\operatorname{Kdim} \mathbf{T}_{i} \leq \ell\right) \Longrightarrow \mathrm{Kdim} \mathbf{T} \leq \ell\right) .
$$

$-\mathrm{Kdim} \mathbf{T}=\sup _{i} \mathrm{Kdim} \mathbf{T}_{i}$ signifie :

$$
\forall \ell \geq-1\left(\&_{i}\left(\mathrm{Kdim} \mathbf{T}_{i} \leq \ell\right) \Longleftrightarrow \mathrm{Kdim} \mathbf{T} \leq \ell\right) .
$$

Le fait que la définition fonctionne aussi bien avec le bord supérieur qu'avec le bord inférieur donne la constatation suivante.

Fait 3.5. - Un treillis distributif et le treillis opposé ont même dimension.

En mathématiques classiques on peut s'en rendre compte directement en considérant les chaînes d'idéaux premiers qui ont pour complémentaires des chaînes de filtres premiers (et vice-versa) : si on identifie les ensembles sous-jacents à $X=\operatorname{Spec} \mathbf{T}$ et $X^{\prime}=\operatorname{Spec} \mathbf{T}^{\circ}$ les relations d'ordre $\leq_{X}$ et $\leq_{X^{\prime}}$ sont opposées.

Notons $\operatorname{Bd}(V, X)$ le bord de $V$ dans $X$ ( $X$ est un espace topologique et $V$ est une partie de $X)$. Alors si $Y$ est un sous espace de $X$ on a $\operatorname{Bd}(V \cap Y, Y) \subseteq \operatorname{Bd}(V, X) \cap Y$, avec égalité si $Y$ est un ouvert. La proposition suivante donne une version duale, constructive, sans points, de cette affirmation.

Proposition 3.6. — (bord de Krull d'un treillis quotient) Soit $\mathbf{L}$ un treillis quotient d'un treillis distributif $\mathbf{T}$. Par abus, nous notons $x$ l'image de $x \in \mathbf{T}$ dans $\mathbf{L}$. Alors $\mathbf{L}_{\mathrm{K}}^{x}$ est un quotient de $\mathbf{T}_{\mathrm{K}}^{x}$ et $\mathbf{L}_{x}^{\mathrm{K}}$ est un quotient de $\mathbf{T}_{x}^{\mathrm{K}}$. En outre si $\mathbf{L}$ est le quotient de $\mathbf{T}$ par un filtre $\mathfrak{f}, \mathbf{L}_{\mathrm{K}}^{x}$ est le quotient de $\mathbf{T}_{\mathrm{K}}^{x}$ par le filtre image de $\mathfrak{f}$ dans $\mathbf{T}_{\mathrm{K}}^{x}$.

Démonstration. - Soient $a, b, x \in \mathbf{T}$, si $a \leq_{\mathbf{T}_{\mathrm{K}}^{x}} b$ il existe $z \in \mathbf{T}$ tel que $x \wedge z \leq_{\mathbf{T}} 0$ et $a \leq_{\mathbf{T}} x \vee z \vee b$. Puisque $\mathbf{L}$ est un quotient de $\mathbf{T}$, on a fortiori $x \wedge z \leq_{\mathbf{L}} 0$ et $a \leq_{\mathbf{L}} x \vee z \vee b$ et donc $a \leq_{\mathbf{L}_{\mathrm{K}}^{x}} b$. Voyons le deuxième point. Notons $\pi: \mathbf{T} \rightarrow \mathbf{L}, \pi_{\mathrm{K}}^{x}: \mathbf{T} \rightarrow \mathbf{T}_{\mathrm{K}}^{x}$ et $\theta: \mathbf{T}_{\mathrm{K}}^{x} \rightarrow \mathbf{L}_{\mathrm{K}}^{x}$ les projections. Il est clair que $\theta\left(\pi_{\mathrm{K}}^{x}(\mathfrak{f})\right)=\{1\}$ de sorte l'on a une factorisation de $\theta$ via $\mathbf{T}_{\mathrm{K}}^{x} /\left(\pi_{\mathrm{K}}^{x}(\mathfrak{f})=1\right)$. Inversement soient $a, b \in \mathbf{T}$ tels que $a \leq_{\mathbf{L}_{\mathrm{K}}^{x}} b$. Nous voulons montrer que $a \leq_{\mathbf{T}_{\mathrm{K}}^{x} /\left(\pi_{\mathrm{K}}^{x}(\mathfrak{f})=1\right)} b$. Par hypothèse il existe $z \in \mathbf{T}$ tel que $a \leq_{\mathbf{L}} x \vee z \vee b$ et $x \wedge z \leq_{\mathbf{L}} 0$. Cela signifie qu'il existe $f_{1}$ et $f_{2} \in \mathfrak{f}$ tels que $a \wedge f_{1} \leq_{\mathbf{T}} b \vee x \vee z$ et $x \wedge z \wedge f_{2} \leq_{\mathbf{T}} 0$. En prenant $f=f_{1} \wedge f_{2}$ et $z^{\prime}=z \wedge f_{2}$ on obtient $a \wedge f \leq_{\mathbf{T}} b \vee x \vee z^{\prime}$ et $x \wedge z^{\prime} \leq_{\mathbf{T}} 0$, c'est-à-dire $a \wedge f \leq_{\mathbf{T}_{\mathrm{K}}^{x}} b$.

Le corollaire suivant donne une version duale, constructive, sans points, du fait suivant : la dimension d'un sous espace spectral est toujours inférieure ou égale à celle de l'espace entier.

Corollaire 3.7. - Si $\mathbf{L}$ est un treillis quotient de $\mathbf{T}$ on a $\mathrm{Kdim} \mathbf{L} \leq \mathrm{Kdim} \mathbf{T}$.

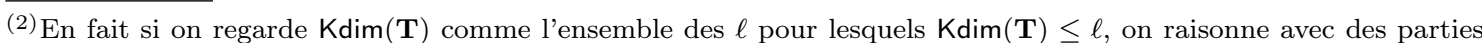
finales (éventuellement vides) de $\mathbb{N} \cup\{-1\}$, la relation d'ordre est alors l'inclusion renversée, la borne supérieure l'intersection et la borne inférieure la réunion. 
Dans la proposition suivante le point 2 est une version duale, constructive, sans points, du fait topologique suivant : la notion de bord est locale. C'est surtout le point 1 qui nous sera utile. En renversant la relation d'ordre on aurait un énoncé analogue pour l'autre bord.

Proposition 3.8. - (caractère local du bord de Krull)

1. Soit $\left(\mathfrak{a}_{i}\right)_{1 \leq i \leq m}$ une famille finie d'idéaux de $\mathbf{T}$, avec $\bigcap_{i=1}^{m} \mathfrak{a}_{i}=\{0\}$. Pour $x \in \mathbf{T}$ notons encore $x$ son image dans $\mathbf{T}_{i}=\mathbf{T} /\left(\mathfrak{a}_{i}=0\right)$. Le bord $\mathbf{T}_{i \mathrm{~K}}^{x}$ peut être vu comme le quotient de $\mathbf{T}_{\mathrm{K}}^{x}$ par un idéal $\mathfrak{b}_{i}$ et on $a: \bigcap_{i=1}^{m} \mathfrak{b}_{i}=\{0\}$.

2. Soit $\left(\mathfrak{f}_{i}\right)_{1 \leq i \leq m}$ une famille finie de filtres de $\mathbf{T}$, avec $\bigcap_{i=1}^{m} \mathfrak{f}_{i}=\{1\}$. Pour $x \in \mathbf{T}$ notons encore $x$ son image dans $\mathbf{T}_{i}=\mathbf{T} /\left(\mathfrak{f}_{i}=1\right)$. Le bord $\mathbf{T}_{i \mathrm{~K}}^{x}$ peut être vu comme le quotient de $\mathbf{T}_{\mathrm{K}}^{x}$ par un filtre $\mathfrak{g}_{i}$ et on $a: \bigcap_{i=1}^{m} \mathfrak{g}_{i}=\{1\}$.

Démonstration. - Voyons le point 1. Considérons la projection $\pi_{i}: \mathbf{T} \rightarrow \mathbf{T}_{i}$ puis la projection $\pi_{i}^{\prime}: \mathbf{T}_{i} \rightarrow \mathbf{T}_{i \mathrm{~K}}^{x}$. La composée $\mathbf{T} \rightarrow \mathbf{T}_{i \mathrm{~K}}^{x}$ montre que $\mathbf{T}_{i \mathrm{~K}}^{x} \simeq \mathbf{T} /\left(\pi_{i}^{-1}\left(\mathrm{~K}_{\mathbf{T}_{i}}^{x}\right)=0\right)$, et l'idéal $\pi_{i}^{-1}\left(\mathrm{~K}_{\mathbf{T}_{i}}^{x}\right)$ contient $\mathrm{K}_{\mathbf{T}}^{x}$. Ceci prouve la première affirmation. Soit maintenant $y \in \mathbf{T}$ tel que pour chaque $i$, $\pi_{i}(y) \in \mathrm{K}_{\mathbf{T}_{i}}^{x}$. Cela revient à dire qu'il existe $b_{i}$ tel que $\pi_{i}(y) \leq \pi_{i}(x) \vee \pi_{i}\left(b_{i}\right)$ et $\pi_{i}\left(b_{i}\right) \wedge \pi_{i}(x)=\pi_{i}(0)$, c'est-à-dire pour un certain $a_{i} \in \mathfrak{a}_{i}: y \leq x \vee a_{i} \vee b_{i}$ et $x \wedge b_{i} \in \mathfrak{a}_{i}$. En prenant $c_{i}=a_{i} \vee b_{i}$ cela fait $y \leq x \vee c_{i}$ et $x \wedge c_{i} \in \mathfrak{a}_{i}$. Enfin avec $c=c_{1} \wedge \cdots \wedge c_{m}$ on obtient $y \leq x \vee c$ avec $c \wedge x=0$. Donc $y \in \mathrm{K}_{\mathbf{T}}^{x}$, et cela montre qu'un $z \in \mathbf{T}_{\mathrm{K}}^{x}$ qui est dans tous les $\mathfrak{b}_{i}$ est nul (car il est la classe d'un $y$ ). Pour le point 2 c'est une conséquence immédiate de la proposition 3.6 et du fait 1.4, qui affirme que tout passage au quotient est un homomorphisme pour les treillis des filtres (en particulier si une intersection finie de filtres est égale à 1 cela reste vrai après passage au quotient).

Corollaire 3.9. - (caractère local de la dimension de Krull)

1. Soit $\left(\mathfrak{a}_{i}\right)_{1 \leq i \leq m}$ une famille finie d'idéaux de $\mathbf{T}$ et $\mathfrak{a}=\bigcap_{i=1}^{m} \mathfrak{a}_{i}$. Alors

$$
\operatorname{Kdim}(\mathbf{T} /(\mathfrak{a}=0))=\sup _{i} \operatorname{Kdim}\left(\mathbf{T} /\left(\mathfrak{a}_{i}=0\right)\right) .
$$

2. Soit $\left(\mathfrak{f}_{i}\right)_{1 \leq i \leq m}$ une famille finie de filtres de $\mathbf{T}$ et $\mathfrak{f}=\bigcap_{i=1}^{m} \mathfrak{f}_{i}$. Alors $\operatorname{Kdim}(\mathbf{T} /(\mathfrak{f}=1))=\sup _{i} \operatorname{Kdim}\left(\mathbf{T} /\left(\mathfrak{f}_{i}=1\right)\right)$.

Démonstration. - Il suffit de prouver le point 1 . En remplaçant $\mathbf{T}$ par $\mathbf{T} /(\mathfrak{a}=0)$ on se ramène au cas où $\mathfrak{a}=0$. Le résultat est clair pour $\ell=-1$. Et la preuve par induction fonctionne grâce à la proposition 3.8 .

On peut aussi donner une preuve directe basée sur la caractérisation 2 (c) dans la définition 3.3.

Notez qu'en mathématiques classiques la caractère local de la dimension de Krull est en général énoncé sous la forme

$$
\operatorname{Kdim}(\mathbf{T})=\sup \{\operatorname{Kdim}(\mathbf{T} /(\mathfrak{f}=1)) \mid \mathfrak{f} \text { filtre premier minimal }\} .
$$

Il s'agit d'une conséquence directe de la définition de la dimension en mathématiques classiques. On peut ensuite en déduire le corollaire 3.9 mais la preuve qu'on obtient ainsi n'est pas constructive.

On obtient également comme dans le théorème 3.1, mais avec une preuve constructive le résultat important suivant, qui nous dit qu'on peut se limiter aux bords construits avec les éléments d'un système générateur de $\mathbf{T}$.

Proposition 3.10. - Soit un treillis distributif $\mathbf{T}$ engendré par une partie $S$ et $\ell$ un entier positif ou nul. Les conditions suivantes sont équivalentes.

1. Le treillis $\mathbf{T}$ est de dimension $\leq \ell$.

2. Pour tout $x \in S$ le bord $\mathbf{T}_{\mathrm{K}}^{x}$ est de dimension $\leq \ell-1$.

3. Pour tout $x \in S$ le bord $\mathbf{T}_{x}^{\mathrm{K}}$ est de dimension $\leq \ell-1$.

4. Pour tous $x_{0}, \ldots, x_{\ell} \in S$ il existe $a_{0}, \ldots, a_{\ell} \in \mathbf{T}$ vérifiant :

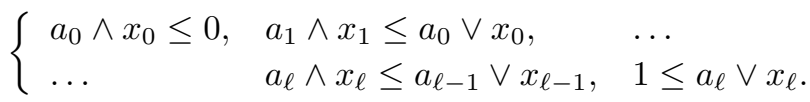


Supposons que $\mathbf{T}$ est une algèbre de Heyting, les conditions précédentes sont aussi équivalentes à

(5) Pour toute suite $x_{0}, \ldots, x_{\ell}$ dans $S$ on a l'égalité

$$
1=x_{\ell} \vee\left(x_{\ell} \rightarrow\left(\cdots\left(x_{1} \vee\left(x_{1} \rightarrow\left(x_{0} \vee \neg x_{0}\right)\right)\right) \cdots\right)\right)
$$

Supposons que $\mathbf{T}$ une algèbre de Brouwer, les conditions précédentes sont aussi équivalentes à

(6) Pour toute suite $x_{0}, \ldots, x_{\ell}$ dans $S$ on a l'égalité

$$
0=x_{0} \wedge\left(x_{0}-\left(x_{1} \wedge\left(x_{1}-\left(\cdots\left(x_{\ell} \wedge\left(1-x_{\ell}\right)\right)\right)\right) \cdots\right)\right)
$$

Démonstration. - $(2) \Leftrightarrow(4)$ par récurrence sur $\ell$, vue la définition du bord.

$(3) \Leftrightarrow(4)$ par récurrence sur $\ell$, vue la définition du bord.

$(2) \Leftrightarrow(5)$ par récurrence sur $\ell$, vue la définition du bord dans le cas d'une algèbre de Heyting.

$(3) \Leftrightarrow(6)$ par récurrence sur $\ell$, vue la définition du bord dans le cas d'une algèbre de Brouwer.

Il reste donc à voir que si (2) est vrai pour $x \in S$, alors (2) est vrai pour tout $x \in \mathbf{T}$. Cela résulte de la proposition 2.7, et des corollaires 3.7 et $3.9:$ par exemple pour tous $x, y \in \mathbf{T}, \mathbf{T}_{\mathrm{K}}^{x \vee y}$ est un quotient de $\mathbf{T} /\left(\mathrm{K}_{\mathbf{T}}^{x} \cap \mathrm{K}_{\mathbf{T}}^{y}\right) \operatorname{donc} \mathrm{Kdim}\left(\mathbf{T}_{\mathrm{K}}^{x \vee y}\right) \leq \sup \left(\mathrm{Kdim} \mathbf{T}_{\mathrm{K}}^{x}, \mathrm{Kdim} \mathbf{T}_{\mathrm{K}}^{y}\right)$.

\subsection{Dimensions et bords de Heitmann. -}

J-dimension de Heitmann pour un treillis distributif. - Nous donnons maintenant la définition constructive de la Jdim de Heitmann, que nous appelons J-dimension de Heitmann du treillis $\mathbf{T}$ (ou de l'espace spectral Spec $\mathbf{T}$ ).

Définition 3.11. - Soit $\mathbf{T}$ un treillis distributif. La J-dimension de Heitmann de $\mathbf{T}$, notée $\operatorname{Jdim} \mathbf{T}$, est la dimension de $\mathrm{Krull}$ de $\mathrm{He}(\mathbf{T})$.

En fait d'un point de vue constructif on s'est contenté de définir, pour tout entier $\ell \geq-1$, la phrase « Jdim $\mathbf{T} \leq \ell » \operatorname{par} « \operatorname{Kdim} \mathrm{He}(\mathbf{T}) \leq \ell »$.

$\mathrm{Du}$ point de vue classique on peut donner la définition directement, à la Heitmann, pour un espace spectral $X$ comme suit : si $M_{X}$ est l'ensemble des points fermés et $J_{X}$ l'adhérence de $M_{X}$ pour la topologie constructible, alors $\operatorname{Jdim} X=\mathrm{Kdim} J_{X}$.

Fait 3.12. - Soit $\mathbf{T}$ un treillis distributif, $\mathbf{T}^{\prime}=\mathbf{T} /\left(\mathrm{J}_{\mathbf{T}}(0)=0\right)$ et $\mathfrak{a}$ un idéal de $\mathbf{T}$.

1. $\operatorname{Jdim}(\mathrm{He}(\mathbf{T}))=\operatorname{Jdim} \mathbf{T}^{\prime}=\operatorname{Jdim} \mathbf{T} \leq \mathrm{Kdim}\left(\mathbf{T}^{\prime}\right) \leq \mathrm{Kdim} \mathbf{T}$.

2. Si $\mathbf{L}=\mathbf{T} /(\mathfrak{a}=0)$ est le quotient de $\mathbf{T}$ par l'idéal $\mathfrak{a}$, alors $\operatorname{Jdim} \mathbf{L} \leq \operatorname{Jdim} \mathbf{T}$.

Démonstration. - Le point 1 est conséquence du point 3 dans le fait 1.12 et le point 2 conséquence du point 4.

Remarque. - L'opération $\mathbf{T} \mapsto \mathrm{J}_{\mathbf{T}}(0)$ n'est pas fonctorielle. Même chose pour $\mathbf{T} \mapsto$ He $\mathbf{T}$. En particulier l'item 4 ne fonctionne plus a priori pour un quotient plus général, par exemple pour un quotient par un filtre. Contrairement à la Kdim la Jdim peut augmenter par passage à un quotient (on a des exemples simples en algèbre commutative).

Exemple. - Voici un exemple dû à Heitmann d'un espace spectral pour lequel

$$
\operatorname{Jdim}(\mathbf{T})<\mathrm{Kdim}\left(\mathbf{T} /\left(\mathrm{J}_{\mathbf{T}}(0)=0\right)\right)
$$

On considère $X=\operatorname{Spec} \mathbb{Z}$ et $Y=\mathbf{n}(n \geq 3)$ on recolle ces deux espaces spectraux en identifiant les deux éléments minimaux (le singleton correspondant est bien dans les deux cas un sous-espace spectral). On obtient un espace $Z=\operatorname{Spec} \mathbf{T}$ avec $M_{Z}=$ Max $\mathbf{T}$ fermé donc égal à $J_{Z}=$ Jspec $\mathbf{T}$ et zéro dimensionnel, tandis que l'unique élément minimal est le seul minorant de $M_{Z}$, donc $\mathrm{J}_{\mathbf{T}}(0)=0$ et $\operatorname{Kdim}\left(\mathbf{T} /\left(\mathrm{J}_{\mathbf{T}}(0)=0\right)\right)=\mathrm{Kdim}(\mathbf{T})=n-2$. 


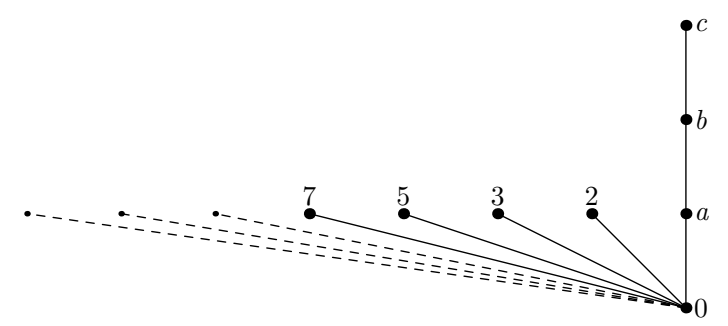

FIG. 1. Exemple de Heitmann

La proposition suivante est signalée par Heitmann dans [15].

Proposition 3.13. - (comparaison de Jspec et de jspec)

- On a toujours jspec $\mathbf{T} \subseteq$ Jspec $\mathbf{T}$.

- Si jspec $\mathbf{T}$ est nœthérien, jspec $\mathbf{T}=$ Jspec $\mathbf{T}$.

Démonstration. - On note $M \subseteq$ Spec $\mathbf{T}$ l'ensembles des idéaux maximaux. On considère un $\mathfrak{p}$ fixé dans Spec $\mathbf{T}$.

Tout d'abord les propriétés suivantes sont successivement équivalentes

$$
\begin{aligned}
& \mathfrak{p} \in \mathrm{jspec} \mathbf{T} \\
& \forall a \in \mathbf{T} \quad\left[\mathfrak{p} \in \mathrm{D}_{\mathbf{T}}(a) \quad \Rightarrow \quad \exists \mathfrak{m} \in\left(M \cap \mathrm{D}_{\mathbf{T}}(a)\right), \mathfrak{p} \subseteq \mathfrak{m}\right] \\
& \forall a \in \mathbf{T} \quad\left[\mathfrak{p} \in \mathrm{D}_{\mathbf{T}}(a) \Rightarrow \quad \exists \mathfrak{m} \in M \cap \mathrm{D}_{\mathbf{T}}(a) \cap \overline{\{\mathfrak{p}\}}\right] \\
& \forall a \in \mathbf{T} \quad\left[\mathfrak{p} \in \mathrm{D}_{\mathbf{T}}(a) \Rightarrow \exists \mathfrak{m} \in\left(M \cap \mathrm{D}_{\mathbf{T}}(a)\right), \forall b \in \mathbf{T} \quad\left(\mathfrak{m} \in \mathrm{D}_{\mathbf{T}}(b) \Rightarrow \mathfrak{p} \in \mathrm{D}_{\mathbf{T}}(b)\right)\right] \\
& \forall a \in \mathbf{T} \quad\left[\mathfrak{p} \in \mathrm{D}_{\mathbf{T}}(a) \Rightarrow \quad \exists \mathfrak{m} \in\left(M \cap \mathrm{D}_{\mathbf{T}}(a)\right), \forall b \in \mathbf{T} \quad\left(\mathfrak{p} \in \mathrm{V}_{\mathbf{T}}(b) \Rightarrow \mathfrak{m} \in \mathrm{V}_{\mathbf{T}}(b)\right)\right] \\
& \forall a \in \mathbf{T} \quad\left[\mathfrak{p} \in \mathrm{D}_{\mathbf{T}}(a) \quad \Rightarrow \quad \exists \mathfrak{m} \in M, \forall b \in \mathbf{T} \quad\left(\mathfrak{p} \in \mathrm{V}_{\mathbf{T}}(b) \Rightarrow \mathfrak{m} \in M \cap \mathrm{D}_{\mathbf{T}}(a) \cap \mathrm{V}_{\mathbf{T}}(b)\right)\right]
\end{aligned}
$$

De même sont équivalentes :

$$
\begin{array}{r}
\mathfrak{p} \in \mathrm{Jspec}_{\mathbf{T}} \\
\forall a, b \in \mathbf{T} \quad\left[\mathfrak{p} \in\left(\mathrm{D}_{\mathbf{T}}(a) \cap \mathrm{V}_{\mathbf{T}}(b)\right) \quad \Rightarrow \quad \exists \mathfrak{m} \in M \cap \mathrm{D}_{\mathbf{T}}(a) \cap \mathrm{V}_{\mathbf{T}}(b)\right]
\end{array}
$$

Ainsi on voit que $(*)$ est plus fort que $(* *)$, puisque dans $(* *), \mathfrak{m}$ peut dépendre de $a$ et $b$ tandis que dans $(*)$ il ne doit dépendre que de $a$.

Si $Z=$ jspec $\mathbf{T}$ est nœethérien l'intersection $\bigcap_{\mathrm{V}_{\mathbf{T}}(b) \ni \mathfrak{p}}\left(Z \cap \mathrm{V}_{\mathbf{T}}(b)\right)$ est égale à un seul $Z \cap \mathrm{V}_{\mathbf{T}}\left(b_{0}\right)$ et $(* *)$ avec ce $b_{0}$ donne $(*)$.

Commentaire. - Comme le fait remarquer Heitmann, les théorèmes connus utilisant le jspec le font toujours sous l'hypothèse « jspec nœethérien ». Il est donc probable que le Jspec soit la seule notion vraiment intéressante. Notez que jspec $\mathbf{T}$ n'est un sous-espace spectral de Spec $\mathbf{T}$ que lorsqu'il est égal à Jspec $\mathbf{T}$.

Remarque. - Donnons la définition de la Jdim complètement mise à plat.

- $\operatorname{Jdim} \mathbf{T} \leq \ell$ signifie $: \forall x_{0}, \ldots, x_{\ell} \in \mathbf{T} \exists a_{0}, \ldots, a_{\ell} \in \mathbf{T}$ vérifiant :

$$
\begin{aligned}
& a_{0} \wedge x_{0} \leq_{\mathrm{He}(\mathbf{T})} 0, \quad a_{1} \wedge x_{1} \leq_{\mathrm{He}(\mathbf{T})} a_{0} \vee x_{0}, \quad \ldots, \\
& \ldots, \quad a_{\ell} \wedge x_{\ell} \leq_{\mathrm{He}(\mathbf{T})} a_{\ell-1} \vee x_{\ell-1}, \quad 1 \leq_{\mathrm{He}(\mathbf{T})} a_{\ell} \vee x_{\ell} .
\end{aligned}
$$

c'est-à-dire encore $\forall x_{0}, \ldots, x_{\ell} \in \mathbf{T} \exists a_{0}, \ldots, a_{\ell} \in \mathbf{T} \quad \forall y \in \mathbf{T}$

$$
\begin{array}{ccc}
\left(a_{0} \wedge x_{0}\right) \vee y=1 & \Rightarrow & y=1 \\
\left(a_{1} \wedge x_{1}\right) \vee y=1 & \Rightarrow & a_{0} \vee x_{0} \vee y=1 \\
\vdots & \vdots & \vdots \\
\left(a_{\ell} \wedge x_{\ell}\right) \vee y=1 & \Rightarrow & a_{\ell-1} \vee x_{\ell-1} \vee y=1 \\
& & a_{\ell} \vee x_{\ell}=1
\end{array}
$$


- En particulier $\operatorname{Jdim} \mathbf{T} \leq 0$ signifie :

$\forall x_{0} \in \mathbf{T} \exists a_{0} \in \mathbf{T} \forall y \in \mathbf{T},\left(\left(a_{0} \wedge x_{0}\right) \vee y=1 \Rightarrow y=1\right)$ et $\left.a_{0} \vee x_{0}=1\right)$.

- Et $\operatorname{Jdim} \mathbf{T} \leq 1$ signifie $: \forall x_{0}, x_{1} \in \mathbf{T} \exists a_{0}, a_{1} \in \mathbf{T} \quad \forall y \in \mathbf{T}$, $\left(\left(a_{0} \wedge x_{0}\right) \vee y=1 \Rightarrow y=1\right)$ et $\left(\left(a_{1} \wedge x_{1}\right) \vee y=1 \Rightarrow a_{0} \vee x_{0} \vee y=1\right)$ et $a_{1} \vee x_{1}=1$.

La dimension de Heitmann pour un treillis distributif. - Bien que Heitmann définisse et utilise dans [15] la dimension Jdim $X$ où $X$ est le spectre d'un anneau commutatif, ses preuves sont en fait implicitement basées sur une notion voisine, mais non équivalente, que nous appellerons la dimension de Heitmann et que nous noterons Hdim.

Nous présentons cette notion directement au niveau des treillis distributifs, où les choses s'expliquent plus simplement.

La dimension Hdim $\mathbf{T}$ est toujours inférieure ou égale à $\operatorname{Jdim} \mathbf{T}$, ce qui fait que les théorèmes établis pour la Hdim seront a fortiori vrais avec la Jdim et avec la Kdim.

Définition 3.14. - Soit $\mathbf{T}$ un treillis distributif et $x \in \mathbf{T}$. On appelle bord de Heitmann de $x$ dans $\mathbf{T}$ le quotient $\mathbf{T}_{\mathrm{H}}^{x} \stackrel{\text { def }}{=} \mathbf{T} /\left(\mathrm{H}_{\mathbf{T}}^{x}=0\right)$, où

$$
\mathrm{H}_{\mathbf{T}}^{x}=\downarrow x \vee\left(\mathrm{J}_{\mathbf{T}}(0): x\right)
$$

On dira aussi que $\mathrm{H}_{\mathbf{T}}^{x}$ est l'idéal bord de Heitmann de $x$ dans $\mathbf{T}$.

Lemme 3.15. - (bord de Krull et bord de Heitmann)

Soit $\mathbf{T}$ un treillis distributif, $\mathbf{T}^{\prime}=\mathbf{T} /\left(\mathrm{J}_{\mathbf{T}}(0)=0\right), \pi: \mathbf{T} \rightarrow \mathbf{T}^{\prime}$ la projection canonique, $x \in \mathbf{T}$ et $\bar{x}=\pi(x)$. Alors on $a$ :

1. $\mathrm{H}_{\mathbf{T}^{\prime}}^{\bar{x}}=\mathrm{K}_{\mathbf{T}^{\prime}}^{\bar{x}}$.

2. $\pi^{-1}\left(\mathrm{~K}_{\mathbf{T}^{\prime}}^{\bar{x}}\right)=\mathrm{H}_{\mathbf{T}}^{x}$ et $\mathbf{T}_{\mathrm{H}}^{\bar{x}} \simeq \mathbf{T}_{\mathrm{K}}^{\prime \bar{x}} \simeq \mathbf{T}_{\mathrm{H}}^{x}$.

Démonstration. - Clair d'après les définitions.

Lemme 3.16. - Soit $\mathbf{L}=\mathbf{T} /(\mathfrak{a}=0)$ un treillis quotient d'un treillis distributif $\mathbf{T}$ par un idéal. Par abus, nous notons $x$ l'image de $x \in \mathbf{T}$ dans $\mathbf{L}$. Alors $\mathbf{L}_{\mathrm{H}}^{x}$ est un quotient de $\mathbf{T}_{\mathrm{H}}^{x}$.

Démonstration. - Notons $\pi: \mathbf{T} \rightarrow \mathbf{L}$ la projection canonique. On veut montrer que si $z \in \mathrm{H}_{\mathbf{T}}^{x}$ alors $\pi(z) \in \mathrm{H}_{\mathbf{L}}^{\pi(x)}$. On suppose donc $z \leq_{\mathbf{T}} x \vee u$ avec $x \wedge u \in \mathrm{J}_{\mathbf{T}}(0)$. Comme $\pi\left(\mathrm{J}_{\mathbf{T}}(0)\right) \subseteq \mathrm{J}_{\mathbf{L}}(0)$, cela donne $\pi(z) \leq_{\mathbf{L}} \pi(x) \vee \pi(u)$ avec $\pi(x) \wedge \pi(u) \in \mathrm{J}_{\mathbf{L}}(0)$, ce qui implique $\pi(z) \in \mathrm{H}_{\mathbf{L}}^{\pi(x)}$.

Remarque. - Le lemme précédent serait faux pour un quotient plus général, par exemple pour un quotient par un filtre. Il reste vrai chaque fois que $\pi\left(\mathrm{J}_{\mathbf{T}}(0)\right) \subseteq \mathrm{J}_{\mathbf{L}}(0)$.

Proposition 3.17. - (comparaison de deux bords à la Heitmann)

On considère pour $x \in \mathbf{T}$ son image $\hat{x} \in \mathrm{He}(\mathbf{T})$.

1. On compare les deux quotients de $\mathbf{T}$ que sont $\mathrm{He}\left(\mathbf{T}_{\mathrm{H}}^{x}\right)$ et $(\mathrm{He}(\mathbf{T}))_{\mathrm{K}}^{\hat{x}}:$ le premier est un quotient du second.

2. Lorsque $\mathrm{He}(\mathbf{T})$ est une algèbre de Heyting, on a égalité.

Démonstration. - Le treillis $(\mathrm{He}(\mathbf{T}))_{\mathrm{K}}^{\hat{x}}$ est un quotient de $\mathbf{T}$ dont la relation de préordre $a \preceq b$ peut etre décrite de la manière suivante :

$$
\exists y \quad\left(x \wedge y \in \mathrm{J}_{\mathbf{T}}(0) \text { et } \forall z[a \vee z=1 \Rightarrow x \vee y \vee b \vee z=1]\right)
$$

Considérons le préordre qui définit $\mathrm{He}\left(\mathbf{T}_{\mathrm{H}}^{x}\right)$ :

$$
\forall u \quad\left(1 \leq_{\mathbf{T}_{\mathrm{H}}^{x}} a \vee u \Rightarrow 1 \leq_{\mathbf{T}_{\mathrm{H}}^{x}} b \vee u\right)
$$

Prouvons que la relation de préordre $\preceq$ entraine la relation de préordre $(* *)$.

On a un $y$ vérifiant $(*)$. On considère un $u$ tel que $1 \leq a \vee u$ dans $\mathbf{T}_{\mathrm{H}}^{x}$ et on cherche à montrer que $1 \leq b \vee u$ dans $\mathbf{T}_{\mathrm{H}}^{x}$.

La relation $1 \leq a \vee u$ dans $\mathbf{T}_{\mathrm{H}}^{x}$ s'ecrit $1 \leq a \vee u \vee x \vee y^{\prime}$ pour un $y^{\prime}$ tel que $y^{\prime} \wedge x \in \mathrm{J}_{\mathbf{T}}(0)$. 
On pose $z=u \vee x \vee y^{\prime}$, on applique $(*)$ et on obtient $x \vee b \vee u \vee\left(y \vee y^{\prime}\right)=1$.

Mais $\left(y \vee y^{\prime}\right) \wedge x \in \mathrm{J}_{\mathbf{T}}(0)$ donc en posant $y^{\prime \prime}=y \vee y^{\prime}$ on a $1 \leq b \vee u \vee x \vee y^{\prime \prime}$ avec $x \wedge y^{\prime \prime} \in \mathrm{J}_{\mathbf{T}}(0)$ c'est-à-dire $1 \leq b \vee u$ dans $\mathbf{T}_{\mathrm{H}}^{x}$.

Voyons le point 2. Nous notons $\pi: \mathbf{T} \rightarrow \mathrm{He}(\mathbf{T})$ la projection canonique. Rappelons (fait 1.12) que $\pi^{-1}(0)=\mathrm{J}_{\mathbf{T}}(0)$ et $\pi^{-1}(1)=\{1\}$. Nous supposons que $\mathrm{He}(\mathbf{T})$ est une algèbre de Heyting. Notons $\widetilde{x}$ un élément de $\mathbf{T}$ tel que $\pi(\widetilde{x})=\pi(x) \rightarrow 0$ dans $\mathrm{He}(\mathbf{T})$. Alors on peut réécrire $(*)$ sous la forme

$$
\forall z[a \vee z=1 \Rightarrow x \vee \widetilde{x} \vee b \vee z=1]
$$

De même $1 \leq \mathbf{T}_{\mathrm{H}}^{x} a \vee u$, qui signifie

$$
\exists y^{\prime}\left(x \wedge y^{\prime} \in \mathrm{J}_{\mathbf{T}}(0) \text { et } 1 \leq a \vee u \vee x \vee y^{\prime}\right),
$$

se réécrit $1 \leq a \vee u \vee x \vee \widetilde{x}$. En conséquence $(* *)$ se réécrit

$$
\forall u[a \vee u \vee x \vee \widetilde{x}=1 \Rightarrow b \vee u \vee x \vee \widetilde{x}]
$$

et il est clair que $\left(*^{\prime}\right)$ et $\left(* *^{\prime}\right)$ sont équivalents.

Définition 3.18. - La dimension de Heitmann d'un treillis distributif $\mathbf{T}$, notée Hdim $\mathbf{T}$, est définie de manière inductive comme suit :

- $\operatorname{Hdim} \mathbf{T}=-1$ si et seulement si $\mathbf{T}=\mathbf{1}$.

- Pour $\ell \geq 0, \operatorname{Hdim} \mathbf{T} \leq \ell$ si et seulement si $: \forall x \in \mathbf{T}, \operatorname{Hdim}\left(\mathbf{T}_{\mathrm{H}}^{x}\right) \leq \ell-1$.

Du lemme 3.16 on déduit par récurrence sur $\ell$, avec la même convention de notation qu'en 3.4 le lemme suivant.

Lemme 3.19. - Si $\mathbf{L}$ est le quotient de $\mathbf{T}$ par un idéal, alors $\operatorname{Hdim} \mathbf{L} \leq \operatorname{Hdim} \mathbf{T}$.

Proposition 3.20. - (comparaison des dimensions Jdim et Hdim)

- On a toujours $\operatorname{Hdim} \mathbf{T} \leq \operatorname{Jdim} \mathbf{T}$.

- Lorsque $\mathrm{He}(\mathbf{T})$ est une algèbre de Heyting, on a égalité : $\operatorname{Hdim} \mathbf{T}=\operatorname{Jdim} \mathbf{T}$.

Démonstration. - Le point 1 se démontre par récurrence sur $\operatorname{Jdim} \mathbf{T}$ à partir du point 1 de la proposition 3.17 .

Pour le point 2, on suppose que $\mathrm{He}(\mathbf{T})$ est une algèbre de Heyting et on fait une récurrence en utilisant le point 2 de la proposition 3.17. Pour que la récurrence fonctionne il faut montrer que $(\mathrm{He}(\mathbf{T}))_{\mathrm{K}}^{\hat{x}}=\mathrm{He}\left(\mathrm{H}_{\mathbf{T}}^{x}\right)$ est également une algèbre de Heyting. Or cela résulte de ce que $(\mathrm{He}(\mathbf{T}))_{\mathrm{K}}^{\hat{x}}$ est un quotient de $\mathrm{He}(\mathbf{T})$ par un idéal principal (puisque $\mathrm{He}(\mathbf{T})$ est une algèbre de Heyting) et du fait 1.14 .

Proposition 3.21. - Notons $\mathbf{T}^{\prime}$ pour le quotient $\mathbf{T} /\left(\mathrm{J}_{\mathbf{T}}(0)=0\right)$.

1. On a toujours $\operatorname{Hdim} \mathbf{T}=\operatorname{Hdim}\left(\mathbf{T}^{\prime}\right)$.

2. $\operatorname{Jdim} \mathbf{T} \leq 0 \Leftrightarrow \operatorname{Hdim} \mathbf{T} \leq 0 \Leftrightarrow \operatorname{Kdim}\left(\mathbf{T}^{\prime}\right) \leq 0$, (c'est-à-dire $\mathbf{T}^{\prime}$ est une algèbre de Boole). C'est le cas lorsque $\mathrm{He}(\mathbf{T})$ est fini.

Démonstration. - Le premier point résulte du lemme 3.15, point 2.

Pour le deuxième point on sait déjà que $\operatorname{Hdim} \mathbf{T} \leq \operatorname{Jdim} \mathbf{T} \leq \operatorname{Kdim} \mathbf{T}^{\prime}$. Le lemme 3.15 , point 1 , prouve que $\operatorname{Hdim} \mathbf{T} \leq 0$ implique $\mathrm{Kdim} \mathbf{T}^{\prime} \leq 0$.

Lorsque $\mathbf{T}$ est fini l'espace Jspec $\mathbf{T}$ est simplement l'ensemble des idéaux maximaux avec pour topologie toutes les parties (puisque les points sont fermés). Par ailleurs le fait 1.12.1 permet de conclure aussi lorsque $\mathrm{He}(\mathbf{T})$ est fini.

Remarque. - Au moins en mathématiques classiques $\mathrm{He}(\mathbf{T})$ est fini si et seulement si l'ensemble $M$ des idéaux maximaux est fini (le cas semi-local en algèbre commutative).

La proposition suivante est l'analogue de la proposition 2.7, en remplaçant le bord de Krull par le bord de Heitmann. 
Proposition 3.22. - Pour tous $x, y \in \mathbf{T}$ les idéaux bords de Heitmann de $x \wedge y$ et $x \vee y$ contiennent l'intersection des idéaux bords de Heitmann de $x$ et $y$, plus précisément :

$$
\mathrm{H}_{\mathbf{T}}^{x} \cap \mathrm{H}_{\mathbf{T}}^{y}=\mathrm{H}_{\mathbf{T}}^{x \vee y} \cap \mathrm{H}_{\mathbf{T}}^{x \wedge y}
$$

Démonstration. — Résulte de la proposition 2.7 et du lemme 3.15.

La proposition suivante est l'analogue du point 1 de la proposition 3.8 .

Proposition 3.23. - On se donne une famille finie $\left(\mathfrak{a}_{i}\right)_{1 \leq i \leq m}$ d'idéaux de $\mathbf{T}$, avec $\bigcap_{i=1}^{m} \mathrm{~J}_{\mathbf{T}}\left(\mathfrak{a}_{i}\right) \subseteq$ $\mathrm{J}_{\mathbf{T}}(0)$ (c'est le cas en particulier si $\bigcap_{i=1}^{m} \mathfrak{a}_{i}=\{0\}$ ). Pour $x \in \mathbf{T}$ notons encore $x$ son image dans $\mathbf{T}_{i}=\mathbf{T} /\left(\mathfrak{a}_{i}=0\right)$. Alors le bord $\mathbf{T}_{i \mathrm{H}}^{x}$ peut être vu comme le quotient de $\mathbf{T}_{\mathrm{H}}^{x}$ par un idéal $\mathfrak{b}_{i}$ et on a : $\bigcap_{i=1}^{m} \mathfrak{b}_{i}=\{0\}$.

Démonstration. - Résulte du lemme 3.15 et de la proposition 3.8, appliquée au treillis $\mathbf{T}^{\prime}=$ $\mathbf{T} /\left(\mathrm{J}_{\mathbf{T}}(0)=0\right)$ et aux idéaux images des $\mathrm{J}_{\mathbf{T}}\left(\mathfrak{a}_{i}\right)$ dans $\mathbf{T}^{\prime}$.

Le corollaire suivant est l'analogue du corollaire 3.9 (avec les mêmes notations qu'en 3.4).

Corollaire 3.24. - Soit $\left(\mathfrak{a}_{i}\right)_{1 \leq i \leq m}$ une famille finie d'idéaux de $\mathbf{T}$ et $\mathfrak{a}=\bigcap_{i=1}^{m} \mathfrak{a}_{i}$. Alors $\operatorname{Hdim}(\mathbf{T} /(\mathfrak{a}=0))=\sup _{i} \operatorname{Hdim}\left(\mathbf{T} /\left(\mathfrak{a}_{i}=0\right)\right)$.

Démonstration. - En remplaçant $\mathbf{T}$ par $\mathbf{T} /(\mathfrak{a}=0)$ on se ramène au cas où $\mathfrak{a}=0$. La chose est claire pour $\ell=-1$. Et la preuve par récurrence fonctionne grâce à la proposition 3.23.

Proposition 3.25. - Soit $S$ un système générateur du treillis distributif $\mathbf{T}$ et $\ell \geq 0$. Les propriétés suivantes sont équivalentes :

1. Pour tout $x \in \mathbf{T}, \operatorname{Hdim}\left(\mathbf{T}_{\mathrm{H}}^{x}\right) \leq \ell-1$.

2. Pour tout $x \in S, \operatorname{Hdim}\left(\mathbf{T}_{\mathrm{H}}^{x}\right) \leq \ell-1$.

Démonstration. - Cela résulte de la proposition 3.22, du lemme 3.16 et du corollaire 3.24 : par exemple pour tous $x, y \in \mathbf{T}$, puisque $\mathrm{H}_{\mathbf{T}}^{x \vee y} \subseteq \mathrm{H}_{\mathbf{T}}^{x} \cap \mathrm{H}_{\mathbf{T}}^{y}$, le treillis $\mathbf{T}_{\mathrm{H}}^{x \vee y}$ est un quotient de $\mathbf{T} /\left(\mathrm{H}_{\mathbf{T}}^{x} \cap\right.$ $\left.\mathrm{H}_{\mathbf{T}}^{y}\right)$ par un idéal, $\operatorname{donc} \operatorname{Hdim}\left(\mathbf{T}_{\mathrm{H}}^{x \vee y}\right) \leq \sup \left(\operatorname{Hdim} \mathbf{T}_{\mathrm{H}}^{x}, \operatorname{Hdim} \mathbf{T}_{\mathrm{H}}^{y}\right)$.

Remarque. - Explicitons encore un peu plus la dimension de Heitmann. Pour ceci nous introduisons «l'idéal bord de Heitmann itéré ». Pour $x_{1}, \ldots, x_{n} \in \mathbf{T}$ nous notons $\mathbf{T}_{\mathrm{H}}\left[x_{1}\right]=\mathbf{T}_{\mathrm{H}}^{x_{1}}$, $\mathbf{T}_{\mathrm{H}}\left[x_{1}, x_{2}\right]=\left(\mathbf{T}_{\mathrm{H}}^{x_{1}}\right)_{\mathrm{H}}^{x_{2}}, \mathbf{T}_{\mathrm{H}}\left[x_{1}, x_{2}, x_{3}\right]=\left(\left(\mathbf{T}_{\mathrm{H}}^{x_{1}}\right)_{\mathrm{H}}^{x_{2}}\right)_{\mathrm{H}}^{x_{3}}$, etc. . les treillis bords quotients successifs, et $\mathrm{H}\left[\mathbf{T} ; x_{1}, \ldots, x_{k}\right]=\mathrm{H}_{\mathbf{T}}\left[x_{1}, \ldots, x_{k}\right]$ désigne le noyau de la projection canonique $\mathbf{T} \rightarrow \mathbf{T}_{\mathrm{H}}\left[x_{1}, \ldots, x_{k}\right]$. Dire que $\operatorname{Hdim} \mathbf{T} \leq \ell$ revient à dire que pour tous $x_{0}, \ldots, x_{\ell} \in \mathbf{T}$ on a $1 \in \mathrm{H}_{\mathbf{T}}\left[x_{0}, \ldots, x_{\ell}\right]$.

Il nous faut donc expliciter les idéaux $\mathrm{H}_{\mathbf{T}}\left[x_{0}, \ldots, x_{\ell}\right]$. Pour ceci nous devons expliciter l'image réciproque $\pi^{-1}(\mathrm{H}[\mathbf{T} /(\mathfrak{a}=0) ; \pi(x)]$ ) (que nous noterons $\mathrm{H}[\mathbf{T}, \mathfrak{a} ; x]$ ) lorsqu'on a une projection $\pi: \mathbf{T} \rightarrow \mathbf{T} /(\mathfrak{a}=0)$.

Par définition on a $y \in \mathrm{H}[\mathbf{T}, \mathfrak{a} ; x]$ si et seulement si $y \leq x \vee z \bmod \mathfrak{a}$ pour un $z$ qui vérifie $\pi(z \wedge x) \in \mathrm{J}_{\mathbf{T} /(\mathfrak{a}=0)}(0)$. Cette dernière condition signifie $: \forall u \in \mathbf{T},(\pi((z \wedge x) \vee u=\pi(1) \Rightarrow \pi(u)=$ $\pi(1)$. Et ceci s'écrit encore

$$
\forall u \in \mathbf{T},((\exists a \in \mathfrak{a}(z \wedge x) \vee u \vee a=1) \Rightarrow(\exists b \in \mathfrak{a} u \vee b=1))
$$

Par ailleurs, $y \leq x \vee z \quad \bmod \mathfrak{a}$ signifie $\exists a^{\prime} \in \mathfrak{a} y \leq x \vee z \vee a^{\prime}$ et la condition $\pi(z \wedge x) \in \mathrm{J}_{\mathbf{T} /(\mathfrak{a}=0)}(0)$ n'est pas changée si on remplace $z$ par $z \vee a^{\prime}$. En bref nous obtenons la condition suivante pour $y \in \mathrm{H}[\mathbf{T}, \mathfrak{a} ; x]:$

$$
\exists z \in \mathbf{T}[y \leq x \vee z \text { et } \forall u \in \mathbf{T},((\exists a \in \mathfrak{a}(z \wedge x) \vee u \vee a=1) \Rightarrow(\exists b \in \mathfrak{a} u \vee b=1))]
$$

On voit donc apparaître une formule d'une complexité logique redoutable. Surtout si on songe que $\exists a \in \mathfrak{a}$ et $\exists b \in \mathfrak{a}$ devront être explicités avec $\mathfrak{a}=\mathrm{H}_{\mathbf{T}}\left[x_{1}, \ldots, x_{k}\right]$ si on veut obtenir un expression pour $y \in \mathrm{H}_{\mathbf{T}}\left[x_{1}, \ldots, x_{k}, x\right]$. Contrairement à l'expression pour $\mathrm{Jdim} \mathbf{T} \leq \ell$ qui ne comportait que deux alternances de quantificateurs quel que soit l'entier $\ell$, on voit pour $\mathrm{Hdim} \leq \ell$ des expressions de plus en plus imbriquées au fur et à mesure que $\ell$ augmente. En fait il se trouve que pour les anneaux 
commutatifs, c'est la Hdim qui fait fonctionner les preuves par récurrence dans les sections 5 et suivantes, et c'est la vraie raison pour laquelle on est amené à introduire cette dimension. Comme elle vérifie $H \operatorname{dim} \mathbf{T} \leq \operatorname{Jdim} \mathbf{T} \leq \mathrm{Kdim}\left(\mathbf{T} /\left(\mathrm{J}_{\mathbf{T}}(0)=0\right)\right)$ on a quand même des moyens raisonnables pour la majorer. Mais on manque d'exemples avec une majoration meilleure que celle $\operatorname{par} \operatorname{Kdim}\left(\mathbf{T} /\left(\mathbf{J}_{\mathbf{T}}(0)=0\right)\right)$.

\section{Dimensions de Krull et Heitmann pour un anneau commutatif}

Dans cette section, A désigne toujours un anneau commutatif.

4.1. Le treillis de Zariski. - Nous rappelons ici l'approche constructive de Joyal [18] pour le spectre d'un anneau commutatif.

Si $J \subseteq \mathbf{A}$, nous notons $\mathcal{I}_{\mathbf{A}}(J)=\langle J\rangle_{\mathbf{A}}$ (ou $\langle J\rangle$ si le contexte est clair) l'idéal engendré par $J$; nous notons $\mathrm{D}_{\mathbf{A}}(J)=\mathrm{D}(\mathbf{A}, J)$ (ou $\mathrm{D}(J)$ si le contexte est clair) le nilradical de l'idéal $\langle J\rangle$ :

$$
\mathrm{D}_{\mathbf{A}}(J)=\sqrt{\langle J\rangle}=\left\{x \in \mathbf{A} \mid \exists m \in \mathbb{N} x^{m} \in\langle J\rangle\right\}
$$

Lorsque $J=\left\{x_{1}, \ldots, x_{n}\right\}$ nous notons $\mathrm{D}_{\mathbf{A}}\left(x_{1}, \ldots, x_{n}\right)=\mathrm{D}\left(\mathbf{A}, x_{1}, \ldots, x_{n}\right)$ pour $\mathrm{D}_{\mathbf{A}}(J)$. Si le contexte est clair, nous abrégeons $\mathrm{D}_{\mathbf{A}}(x)$ en $\widetilde{x}$. Les $\widetilde{x}$ forment un système générateur de Zar $\mathbf{A}$, stable par $\wedge$.

Par définition le treillis de Zariski de $\mathbf{A}$, noté Zar A, a pour éléments les radicaux d'idéaux de type fini : ce sont donc les $\mathrm{D}_{\mathbf{A}}\left(x_{1}, \ldots, x_{n}\right)$, c'est-à-dire les $\mathrm{D}_{\mathbf{A}}(\mathfrak{a})$ pour les idéaux de type fini $\mathfrak{a}$. La relation d'ordre est l'inclusion, le inf et le sup sont donnés par

$$
\mathrm{D}_{\mathbf{A}}\left(\mathfrak{a}_{1}\right) \wedge \mathrm{D}_{\mathbf{A}}\left(\mathfrak{a}_{2}\right)=\mathrm{D}_{\mathbf{A}}\left(\mathfrak{a}_{1} \mathfrak{a}_{2}\right) \quad \text { et } \quad \mathrm{D}_{\mathbf{A}}\left(\mathfrak{a}_{1}\right) \vee \mathrm{D}_{\mathbf{A}}\left(\mathfrak{a}_{2}\right)=\mathrm{D}_{\mathbf{A}}\left(\mathfrak{a}_{1}+\mathfrak{a}_{2}\right)
$$

Le treillis de Zariski de $\mathbf{A}$ est un treillis distributif, et

$$
\mathrm{D}_{\mathbf{A}}\left(x_{1}, \ldots, x_{n}\right)=\widetilde{x_{1}} \vee \cdots \vee \widetilde{x_{n}}
$$

Si $J \subseteq \mathbf{A}$ nous notons $\widetilde{J}=\{\widetilde{x} \mid x \in J\} \subseteq$ Zar A. Soient $U$ et $J$ deux familles finies dans $\mathbf{A}$, on a

$$
\bigwedge \widetilde{U} \leq_{\text {Zara }} \bigvee \widetilde{J} \Longleftrightarrow \prod_{u \in U} u \in \sqrt{\langle J\rangle} \Longleftrightarrow \mathcal{M}(U) \cap\langle J\rangle \neq \emptyset
$$

où $\mathcal{M}(U)$ est le monoïde multiplicatif engendré par $U$.

Cela suffit à décrire le treillis Zar A. Plus précisément on a [2, 4] :

Proposition 4.1. - Le treillis Zar A est (à isomorphisme près) le treillis engendré par des symboles $\mathrm{D}_{\mathbf{A}}(a)$ soumis aux relations suivantes

$$
\begin{array}{ll}
\mathrm{D}_{\mathbf{A}}\left(0_{\mathbf{A}}\right)=0 & \mathrm{D}_{\mathbf{A}}\left(1_{\mathbf{A}}\right)=1 \\
\mathrm{D}_{\mathbf{A}}(x+y) \leq \mathrm{D}_{\mathbf{A}}(x) \vee \mathrm{D}_{\mathbf{A}}(y) & \mathrm{D}_{\mathbf{A}}(x y)=\mathrm{D}_{\mathbf{A}}(x) \wedge \mathrm{D}_{\mathbf{A}}(y)
\end{array}
$$

L'opération Zar est un foncteur de la catégorie des anneaux commutatifs vers celle des treillis distributifs. Notez que via ce foncteur la projection $\mathbf{A} \rightarrow \mathbf{A} / \mathrm{D}_{\mathbf{A}}(0)$ donne un isomorphisme $\operatorname{Zar} \mathbf{A} \simeq \operatorname{Zar}\left(\mathbf{A} / \mathrm{D}_{\mathbf{A}}(0)\right)$. On a $\operatorname{Zar} \mathbf{A}=\mathbf{1}$ si et seulement si $1_{\mathbf{A}}=0_{\mathbf{A}}$.

Un théorème important de Hochster [16] affirme que tout espace spectral est homéomorphe au spectre d'un anneau commutatif. Une version sans point du théorème de Hochster est : tout treillis distributif est isomorphe au treillis de Zariski d'un anneau commutatif (pour une preuve non constructive voir [1]).

4.2. Idéaux, filtres et quotients de Zar A. - Rappelons qu'en mathématiques classiques le spectre de Zariski Spec A d'un anneau commutatif est un espace topologique dont les points sont les idéaux premiers de l'anneau et dont la topologie est définie par la base d'ouverts formée par les $\mathfrak{D}_{\mathbf{A}}(a)=\{\mathfrak{p} \in \operatorname{Spec} \mathbf{A} \mid a \notin \mathfrak{p}\}$. On note aussi $\mathfrak{D}_{\mathbf{A}}\left(x_{1}, \ldots, x_{n}\right)$ pour $\mathfrak{D}_{\mathbf{A}}\left(x_{1}\right) \cup \cdots \cup \mathfrak{D}_{\mathbf{A}}\left(x_{n}\right)$. 
Idéaux de A et de Zar A. - Nous disons qu'un idéal $\mathfrak{J}$ est radical si $\mathfrak{J}=\sqrt{\mathfrak{J}}$. En mathématiques classiques, tout idéal radical est l'intersection des idéaux premiers qui le contiennent.

Introduisons la notation (lorsque $J \subseteq \mathbf{A}$ )

$$
\mathcal{I Z}_{\mathbf{A}}(J)=\mathcal{I}_{\text {Zar } \mathbf{A}}(\widetilde{J})
$$

pour l'idéal de Zar A engendré par $\widetilde{J}$. En particulier

$$
\mathcal{I Z}_{\mathbf{A}}\left(x_{1}, \ldots, x_{n}\right)=\downarrow \mathrm{D}_{\mathbf{A}}\left(x_{1}, \ldots, x_{n}\right)=\downarrow\left(\widetilde{x_{1}} \vee \cdots \vee \widetilde{x_{n}}\right) .
$$

On a $\mathcal{I Z}_{\mathbf{A}}(J)=\mathcal{I Z}_{\mathbf{A}}(\sqrt{\langle J\rangle})$, et on établit facilement le fait fondamental suivant.

Fait 4.2. -

- L'application $\mathfrak{a} \mapsto \mathcal{I Z}_{\mathbf{A}}(\mathfrak{a})$ définit un isomorphisme du treillis des idéaux radicaux de $\mathbf{A}$ vers le treillis des idéaux de Zar $\mathbf{A}$.

- Par restriction les idéaux premiers (resp. les idéaux maximaux) de l'anneau $\mathbf{A}$ et ceux du treillis distributif Zar A sont également en correspondance naturelle bijective.

- Pour tout anneau commutatif A, Spec A (au sens des anneaux commutatifs) s'identifie à Spec(Zar A) (au sens des treillis distributifs).

Remarque. - En mathématiques classiques on a un isomorphisme entre le treillis Zar $\mathbf{A}$ et le treillis des ouverts quasi-compacts de $\operatorname{Spec} \mathbf{A}$. On peut alors identifier $\mathrm{D}_{\mathbf{A}}\left(x_{1}, \ldots, x_{n}\right)$ (qui est un élément de Zar $\mathbf{A}), \mathrm{D}_{\text {Zar } \mathbf{A}}\left(\mathrm{D}_{\mathbf{A}}\left(x_{1}, \ldots, x_{n}\right)\right.$ ) (qui est un ouvert quasi-compact de $\operatorname{Spec}(\operatorname{Zar} \mathbf{A})$ ) et $\mathfrak{D}_{\mathbf{A}}\left(x_{1}, \ldots, x_{n}\right)$ (qui est un ouvert quasi-compact de $\mathrm{Spec} \mathbf{A}$ ). Du point de vue constructif, on considère Spec $\mathbf{A}$ comme un « espace topologique sans point », c'est-à-dire un espace défini uniquement à travers une base d'ouverts, et les « identifications » ci-dessus sont de pures identités.

On a aussi facilement :

Fait 4.3. - (quotients)

Si $J \subseteq \mathbf{A}$, alors $\operatorname{Zar}(\mathbf{A} /\langle J\rangle) \simeq \operatorname{Zar}\left(\mathbf{A} / \mathrm{D}_{\mathbf{A}}(J)\right) \simeq \operatorname{Zar}(\mathbf{A}) /\left(\mathcal{I Z}_{\mathbf{A}}(J)=0\right)$.

Fait 4.4. - (transporteurs)

Soient $\mathfrak{A}$ et $\mathfrak{B}$ des idéaux de $\mathbf{A}, \mathfrak{a}=\mathrm{D}_{\mathbf{A}}(\mathfrak{A})$ et $\mathfrak{b}=\mathrm{D}_{\mathbf{A}}(\mathfrak{B})$. Alors $\mathfrak{a}: \mathfrak{b}=\mathfrak{a}: \mathfrak{B}$ est un idéal radical de $\mathbf{A}$ et dans $\operatorname{Zar} \mathbf{A}$ on a $\mathcal{I Z}_{\mathbf{A}}(\mathfrak{a}): \mathcal{I Z}_{\mathbf{A}}(\mathfrak{b})=\mathcal{I Z}_{\mathbf{A}}(\mathfrak{a}: \mathfrak{b})$.

Fait 4.5. - (recouvrement par des idéaux)

Soit $\mathfrak{a}_{i}$ une famille finie d'idéaux de $\mathbf{A}$. Les $\mathcal{I Z}_{\mathbf{A}}\left(\mathfrak{a}_{i}\right)$ recouvrent Zar $\mathbf{A}$ (c'est-à-dire leur intersection est réduite à 0$)$ si et seulement si $\bigcap_{i} \mathfrak{a}_{i} \subseteq \mathrm{D}_{\mathbf{A}}(0)$.

En mathématiques classiques le treillis de Zariski de $\mathbf{A}$ est nœthérien (ce qui revient à dire que Spec $\mathbf{A}$ est nothérien) si et seulement si tout idéal $\mathrm{D}_{\mathbf{A}}(\mathfrak{J})$ est « radicalement de type fini », c'est-à-dire est un élément de Zar $\mathbf{A}$ : un élément de la forme $\mathrm{D}_{\mathbf{A}}\left(x_{1}, \ldots, x_{n}\right)$.

Le treillis Zar $\mathbf{A}=\mathbf{T}$ est une algèbre de Heyting si et seulement si

$$
\forall \mathfrak{a}, \mathfrak{b} \in \mathbf{T}, \quad(\mathfrak{a}: \mathfrak{b}) \in \mathbf{T} .
$$

Le résultat suivant est important en mathématiques constructives.

Proposition 4.6. - (cf. [4]) Si A est un anneau nœthérien cohérent Zar A est une algèbre de Heyting. Si en outre $\mathbf{A}$ est fortement discret, la relation d'ordre dans Zar $\mathbf{A}$ est décidable (on dit alors que le treillis est discret).

Filtres de A et de Zar A. - Un filtre dans un anneau commutatif est un monoïde $\mathfrak{F}$ qui vérifie $x y \in \mathfrak{F} \Rightarrow x \in \mathfrak{F}$. Un filtre premier est un filtre qui vérifie $x+y \in \mathfrak{F} \Rightarrow x \in \mathfrak{F}$ ou $y \in \mathfrak{F}$ (c'est le complémentaire d'un idéal premier).

Pour $x \in \mathbf{A}$ le filtre $\uparrow \widetilde{x}$ de Zar $\mathbf{A}$ est noté $\mathcal{F}_{\mathbf{A}}(x)$. Plus généralement pour $S \subseteq \mathbf{A}$ on note $\mathcal{F Z}_{\mathbf{A}}(S)$ le filtre de $\operatorname{Zar} \mathbf{A}$ :

$$
\mathcal{F Z}_{\mathbf{A}}(S)=\{x \in \operatorname{Zar} \mathbf{A} \mid \exists y \in \mathcal{M}(S) x \geq \widetilde{y}\} .
$$


On a aussi $\mathcal{F Z}_{\mathbf{A}}(S)=\mathcal{F} \mathcal{Z}_{\mathbf{A}}(\mathfrak{F})$ où $\mathfrak{F}$ est le filtre de $\mathbf{A}$ engendré par $S$.

Les faits suivants sont faciles.

Fait 4.7. - L'application $\mathfrak{f} \mapsto \mathcal{F Z}_{\mathbf{A}}(\mathfrak{f})$ établit une correspondance injective croissante des filtres de $\mathbf{A}$ vers les filtres de Zar $\mathbf{A}$, et un sup fini (le sup de $\mathfrak{f}_{1}$ et $\mathfrak{f}_{2}$ est engendré par les $f_{1} f_{2}$ où $f_{i} \in \mathfrak{f}_{i}$ ) donne pour image le sup fini des filtres images. Cette correspondance $\mathcal{F}_{\mathcal{A}}$ se restreint en une bijection entre les filtres premiers de $\mathbf{A}$ et ceux de Zar $\mathbf{A}$.

Notez cependant que le filtre principal de Zar $\mathbf{A}$ engendré par $\widetilde{a_{1}} \vee \cdots \vee \widetilde{a_{n}}$ (c'est-à-dire l'intersection des filtres $\uparrow \widetilde{a_{i}}$ ), ne correspond en général à aucun filtre de $\mathbf{A}$.

Fait 4.8. - (localisés)

Soit $S$ un monoïde de $\mathbf{A}, \mathfrak{F}$ le filtre engendré par $S$, et $\mathfrak{f}=\mathcal{F} \mathcal{Z}_{\mathbf{A}}(S)=\mathcal{F} \mathcal{Z}_{\mathbf{A}}(\mathfrak{F})$.

Alors $S^{-1} \mathbf{A}=\mathbf{A}_{S}=\mathbf{A}_{\mathfrak{F}}$ et $\operatorname{Zar}\left(\mathbf{A}_{S}\right) \simeq \operatorname{Zar}(\mathbf{A}) /(\mathfrak{f}=1)$.

Fait 4.9. - (filtre complémentaire)

Soit $x \in \mathbf{A}$, le filtre $1_{Z a r} \mathbf{A} \backslash \mathcal{F} \mathcal{Z}_{\mathbf{A}}(x)$ est égal à $\mathcal{F Z}_{\mathbf{A}}(1+x \mathbf{A})$.

Fait 4.10. - (recouvrement par des filtres)

Soit $\left(S_{i}\right)_{1 \leq i \leq n}$ une famille finie de monoüdes de $\mathbf{A}$.

1. Les filtres $\mathcal{F Z}_{\mathbf{A}}\left(S_{i}\right)$ recouvrent Zar $\mathbf{A}$ (c'est-à-dire leur intersection est réduite à $\{1\}$ ) si et seulement si les monö̈des $S_{i}$ sont comaximaux c'est-à-dire que pour tous $x_{i} \in S_{i}$ on a $\left\langle x_{1}, \ldots, x_{n}\right\rangle=\langle 1\rangle$.

2. Plus généralement on a $\mathcal{F} \mathcal{Z}_{\mathbf{A}}\left(S_{1}\right) \cap \cdots \cap \mathcal{F}_{\mathbf{A}}\left(S_{n}\right) \subseteq \mathcal{F Z}_{\mathbf{A}}(S)$ si et seulement si pour tous $x_{i} \in S_{i}$ il existe $x \in S$ tel que $x \in\left\langle x_{1}, \ldots, x_{n}\right\rangle$.

4.3. Le treillis de Heitmann. - Dans un anneau commutatif, le radical de Jacobson d'un idéal $\mathfrak{J}$ est (du point de vue des mathématiques classiques) l'intersection des idéaux maximaux qui contiennent $\mathfrak{J}$. On le note $\mathrm{J}_{\mathbf{A}}(\mathfrak{J})=\mathrm{J}(\mathbf{A}, \mathfrak{J})$, ou $\mathrm{J}(\mathfrak{J})$ si le contexte est clair. En mathématiques constructives on utilise la définition suivante, classiquement équivalente :

$$
\mathrm{J}_{\mathbf{A}}(\mathfrak{J}) \stackrel{\text { def }}{=}\{x \in \mathbf{A} \mid \forall y \in \mathbf{A}, \quad 1+x y \text { est inversible modulo } \mathfrak{J}\}
$$

On notera $\mathrm{J}_{\mathbf{A}}\left(x_{1}, \ldots, x_{n}\right)=\mathrm{J}\left(\mathbf{A}, x_{1}, \ldots, x_{n}\right)$ pour $\mathrm{J}_{\mathbf{A}}\left(\left\langle x_{1}, \ldots, x_{n}\right\rangle\right)$. L'idéal $\mathrm{J}_{\mathbf{A}}(0)$ est appelé le radical de Jacobson de l'anneau $\mathbf{A}$.

Définition 4.11. - Le treillis $\mathrm{He}(\mathrm{Zar} \mathbf{A})$ s'appelle treillis de Heitmann de l'anneau commutatif A et se note Heit $\mathbf{A}$.

En mathématiques classiques, vu le fait 4.2 et vue la définition du radical de Jacobson via les intersections d'idéaux maximaux, le fait suivant, qui conduit à une interprétation simple du treillis Heit A, est évident. Nous sommes néanmoins intéressés par une preuve constructive directe.

Fait 4.12. - (radical de Jacobson)

La correspondance bijective $\mathcal{I Z}_{\mathbf{A}}$ préserve le passage au radical de Jacobson. Autrement dit si $\mathfrak{J}$ est un idéal de $\mathbf{A}$ et $\mathfrak{j}=\mathcal{I Z}_{\mathbf{A}}(\mathfrak{J})$, alors $\mathrm{J}_{\mathrm{Zar} \mathbf{A}}(\mathfrak{j})=\mathcal{I Z}_{\mathbf{A}}\left(\mathrm{J}_{\mathbf{A}}(\mathfrak{J})\right)$.

Démonstration. - Il suffit de montrer que pour tout $x \in \mathbf{A}, \widetilde{x} \in \mathrm{J}_{\mathrm{Zar} \mathbf{A}}(\mathrm{j})$ si et seulement si $\widetilde{x} \in \mathcal{I Z}_{\mathbf{A}}\left(\mathrm{J}_{\mathbf{A}}(\mathfrak{J})\right)$. Puisque $\mathrm{J}_{\mathbf{A}}(\mathfrak{J})$ est un idéal radical, on cherche donc à montrer l'équivalence

$$
\forall x \in \mathbf{A} \quad\left(\widetilde{x} \in \mathrm{J}_{\text {Zar } \mathbf{A}}(\mathfrak{j}) \Leftrightarrow x \in \mathrm{J}_{\mathbf{A}}(\mathfrak{J})\right)
$$

Par définition $\widetilde{x} \in \mathrm{J}_{\mathrm{Zar} \mathbf{A}}(\mathfrak{j})$ signifie

$$
\forall y \in \operatorname{Zar} \mathbf{A} \quad(\widetilde{x} \vee y=1 \text { Zar } \mathbf{A} \Rightarrow \exists z \in \mathfrak{j} z \vee y=1 \text { Zar } \mathbf{A})
$$

c'est-à-dire encore puisque tout $y \in$ Zar $\mathbf{A}$ est de la forme $\mathrm{D}_{\mathbf{A}}\left(y_{1}, \ldots, y_{k}\right)$,

$$
\forall y_{1}, \ldots, y_{k} \in \mathbf{A} \quad\left(\left\langle x, y_{1}, \ldots, y_{k}\right\rangle=1_{\mathbf{A}} \Rightarrow \exists z \in \mathfrak{j} z \vee \mathrm{D}_{\mathbf{A}}\left(y_{1}, \ldots, y_{k}\right)=1_{\text {Zar } \mathbf{A}}\right)
$$


ceci est immédiatement équivalent à

$$
\forall y_{1}, \ldots, y_{k} \in \mathbf{A} \quad\left(\left\langle x, y_{1}, \ldots, y_{k}\right\rangle=1_{\mathbf{A}} \Rightarrow \exists u \in \mathfrak{J} \quad\left\langle u, y_{1}, \ldots, y_{k}\right\rangle=1_{\mathbf{A}}\right)
$$

puis à

$$
\forall y \in \mathbf{A}\left(\langle x, y\rangle=1_{\mathbf{A}} \Rightarrow \exists u \in \mathfrak{J} \quad\langle u, y\rangle=1_{\mathbf{A}}\right)
$$

ou encore à : tout $y \in \mathbf{A}$ de la forme $1+x a$ est inversible modulo $\mathfrak{J}$. C'est-à-dire $x \in \mathrm{J}_{\mathbf{A}}(\mathfrak{J})$.

Corollaire 4.13. - Soient $\mathfrak{j}_{1}$ et $\mathfrak{j}_{2}$ deux idéaux de type fini de $\mathbf{A}$. Les éléments $\mathrm{D}_{\mathbf{A}}\left(\mathfrak{j}_{1}\right)$ et $\mathrm{D}_{\mathbf{A}}\left(\mathfrak{j}_{2}\right)$ de Zar $\mathbf{A}$ sont égaux dans le quotient Heit $\mathbf{A}$ si et seulement si $\mathrm{J}_{\mathbf{A}}\left(\mathfrak{j}_{1}\right)=\mathrm{J}_{\mathbf{A}}\left(\mathfrak{j}_{2}\right)$. En conséquence Heit $\mathbf{A}$ s'identifie à l'ensemble des $\mathrm{J}_{\mathbf{A}}\left(x_{1}, \ldots, x_{n}\right)$, avec $\mathrm{J}_{\mathbf{A}}\left(\mathfrak{j}_{1}\right) \wedge \mathrm{J}_{\mathbf{A}}\left(\mathfrak{j}_{2}\right)=\mathrm{J}_{\mathbf{A}}\left(\mathfrak{j}_{1} \mathfrak{j}_{2}\right)$ et $\mathrm{J}_{\mathbf{A}}\left(\mathfrak{j}_{1}\right) \vee$ $\mathrm{J}_{\mathbf{A}}\left(\mathfrak{j}_{2}\right)=\mathrm{J}_{\mathbf{A}}\left(\mathfrak{j}_{1}+\mathfrak{j}_{2}\right)$.

Remarques. -

1. $\mathrm{Vu}$ les bonnes propriétés de la correspondance $\mathcal{I Z}_{\mathbf{A}}$, avec $\mathbf{T}=$ Zar $\mathbf{A}$, on a l'isomorphisme $\operatorname{Zar}\left(\mathbf{A} / \mathrm{J}_{\mathbf{A}}(0)\right) \simeq \mathbf{T} /\left(\mathrm{J}_{\mathbf{T}}(0)=0\right)$. Par contre il ne semble pas qu'il y ait une $\mathbf{A}$-algèbre $\mathbf{B}$ naturellement attachée à $\mathbf{A}$ pour laquelle on ait $\operatorname{Zar} \mathbf{B} \simeq \mathrm{He}(\operatorname{Zar} \mathbf{A})$.

2. Notez que, en général $\mathrm{J}_{\mathbf{A}}\left(x_{1}, \ldots, x_{n}\right)$ est un idéal radical mais pas le radical d'un idéal de type fini.

3. On voit aussi facilement que $\mathrm{J}_{\mathbf{A}}\left(\mathfrak{j}_{1}\right) \wedge \mathrm{J}_{\mathbf{A}}\left(\mathfrak{j}_{2}\right)=\mathrm{J}_{\mathbf{A}}\left(\mathfrak{j}_{1}\right) \cap \mathrm{J}_{\mathbf{A}}\left(\mathfrak{j}_{2}\right)=\mathrm{J}_{\mathbf{A}}\left(\mathfrak{j}_{1} \cap \mathfrak{j}_{2}\right)$ (cela résulte d'ailleurs du lemme 1.11). Il peut sembler surprenant que $J_{\mathbf{A}}\left(\mathfrak{j}_{1}\right) \cap J_{\mathbf{A}}\left(\mathfrak{j}_{2}\right)=J_{\mathbf{A}}\left(\mathfrak{j}_{1} \mathfrak{j}_{2}\right)$ (c'est a priori moins clair que pour les $\left.\mathrm{D}_{\mathbf{A}}\right)$. Voici le calcul élémentaire qui (re)démontre ce fait. On a $x \in \mathrm{J}_{\mathbf{A}}\left(\mathfrak{j}_{1}\right)$ si et seulement si $\forall y(1+x y)$ est inversible modulo $\mathfrak{j}_{1}$, et $x \in \mathrm{J}_{\mathbf{A}}\left(\mathfrak{j}_{2}\right)$ si et seulement si $\forall y(1+x y)$ est inversible modulo $\mathfrak{j}_{2}$. Mais si $a=1+x y$ est inversible modulo $\mathfrak{j}_{1}$ et $\mathfrak{j}_{2}$, il est inversible modulo leur produit : en effet $1+a a_{1} \in \mathfrak{j}_{1}$ et $1+a a_{2} \in \mathfrak{j}_{2}$ impliquent que $\left(1+a a_{1}\right)\left(1+a a_{2}\right)$, qui se réécrit $1+a a^{\prime}$, est dans $\mathfrak{j}_{1} \mathfrak{j}_{2}$.

4.4. Dimension et bords de Krull. — En mathématiques constructives on donne la définition suivante.

Définition 4.14. - La dimension de Krull d'un anneau commutatif est la dimension de Krull de son treillis de Zariski.

Vus le fait 4.2 et le théorème 3.1, il s'agit d'une définition équivalente à la définition usuelle en mathématiques classiques.

Définition 4.15. - Soit $\mathbf{A}$ un anneau commutatif, $x \in \mathbf{A}$ et $\mathbf{j}$ un idéal de type fini.

1. Le bord supérieur de Krull de $\mathbf{j}$ dans $\mathbf{A}$ est l'anneau quotient $\mathbf{A}_{\mathrm{K}}^{\mathfrak{j}}:=\mathbf{A} / \mathrm{K}_{\mathbf{A}}(\mathfrak{j})$ où

$$
K_{\mathbf{A}}(\mathfrak{j}):=\mathfrak{j}+\left(D_{\mathbf{A}}(0): \mathfrak{j}\right)
$$

On note aussi $\mathbf{A}_{\mathrm{K}}^{x}=\mathbf{A}_{\mathrm{K}}^{x \mathbf{A}}$ et on l'appelle le bord supérieur de $x$ dans $\mathbf{A}$. On dira aussi que $\mathrm{K}_{\mathbf{A}}(\mathfrak{j})$ est l'idéal bord de Krull de $\mathbf{j}$. On notera aussi $\mathrm{K}_{\mathbf{A}}\left(y_{1}, \ldots, y_{n}\right)$ pour $\mathrm{K}_{\mathbf{A}}\left(\left\langle y_{1}, \ldots, y_{n}\right\rangle\right)$ et $\mathrm{K}_{\mathbf{A}}^{x}$ pour $\mathrm{K}_{\mathbf{A}}(x)$.

2. Le bord inférieur de Krull de $x$ dans $\mathbf{A}$ est l'anneau localisé $\mathbf{A}_{x}^{\mathrm{K}}:=\mathbf{A}_{\mathrm{S}_{x}^{\mathrm{K}}}$ où $\mathrm{S}_{x}^{\mathrm{K}}=x^{\mathbb{N}}(1+x \mathbf{A})$. On dira aussi que le monoïde $x^{\mathbb{N}}(1+x \mathbf{A})$ est le monö̈de bord de Krull de $x$.

Ainsi un élément arbitraire de $\mathrm{K}_{\mathbf{A}}\left(y_{1}, \ldots, y_{n}\right)$ s'écrit $\sum_{i} a_{i} y_{i}+b$ avec tous les $b y_{i}$ nilpotents.

Proposition 4.16. - Soit $x \in \mathbf{A}$ et $\mathbf{j}=\left\langle j_{1}, \ldots, j_{n}\right\rangle$ un idéal de type fini. Considérons $\widetilde{x} \in$ Zar $\mathbf{A}$ et $\varphi=\mathrm{D}_{\mathbf{A}}(\mathfrak{j})=\tilde{j_{1}} \vee \cdots \vee \widetilde{j_{n}} \in \operatorname{Zar} \mathbf{A}$. Alors :

1. L'idéal bord de Krull de $\varphi=\mathrm{D}_{\mathbf{A}}(\mathfrak{j})$ dans $Z$ Zar $\mathbf{A}, \mathrm{K}_{\mathrm{Zar} \mathbf{A}}$, est égal à $\mathcal{I Z}_{\mathbf{A}}\left(\mathrm{K}_{\mathbf{A}}(\mathfrak{j})\right)$. En conséquence $(\operatorname{Zar} \mathbf{A})_{\mathrm{K}}^{\varphi}$ s'identifie naturellement avec $\operatorname{Zar}\left(\mathbf{A}_{\mathrm{K}}^{\mathfrak{j}}\right)$.

2. Le filtre bord de Krull de $\widetilde{x}$ dans $\mathrm{Zar} \mathbf{A}, \mathrm{K}_{\widetilde{x}}^{\mathrm{Zar} \mathbf{A}}$, est égal à $\mathcal{F} \mathcal{Z}\left(\mathrm{S}_{x}^{\mathrm{K}}\right)$. En conséquence $\left.(\mathrm{Zar} \mathbf{A})\right)_{\widetilde{x}}^{\mathrm{K}}$ s'identifie naturellement avec $\operatorname{Zar}\left(\mathbf{A}_{x}^{\mathrm{K}}\right)$. 
Démonstration. - Pour l'idéal bord, on a par définition $\mathrm{K}_{\mathrm{Zar} \mathbf{A}}=\mathrm{K}_{\mathrm{Zar} \mathbf{A}}\left(\mathrm{D}_{\mathbf{A}} \varphi\right)=\mathrm{D}_{\mathbf{A}} \varphi \vee\left(\mathrm{D}_{\mathbf{A}}(0)\right.$ : $\left.\mathrm{D}_{\mathbf{A}} \varphi\right)$. D'après les faits 4.2 et 4.4 , il est égal à $\mathcal{I Z}_{\mathbf{A}}\left(\varphi+\left(\mathrm{D}_{\mathbf{A}}(0): \varphi\right)\right)$ et aussi à $\mathcal{I}_{\mathbf{A}}\left(\mathfrak{j}+\left(\mathrm{D}_{\mathbf{A}}(0)\right.\right.$ : $\mathfrak{j}))=\mathcal{I Z}_{\mathbf{A}}\left(\mathrm{K}_{\mathbf{A}}^{\mathfrak{j}}\right)$. Enfin pour les passages au quotient on applique le fait 4.3.

Pour le filtre bord de Krull, cela fonctionne de la même manière en utilisant les faits 4.7 et 4.9 puis en passant au treillis quotient avec le fait 4.8 .

Comme corollaire des propositions 3.10 et 4.16 on obtient l'analogue suivant du théorème 3.1 , dans une version entièrement constructive. Rappelons que la dimension de Krull d'un anneau est -1 si et seulement si l'anneau est trivial (i.e., $1_{\mathbf{A}}=0_{\mathbf{A}}$ ).

Théorème 4.17. - Pour un anneau commutatif $\mathbf{A}$ et un entier $\ell \geq 0$ les propriétés suivantes sont équivalentes :

1. La dimension de Krull de $\mathbf{A}$ est $\leq \ell$.

2. Pour tout $x \in \mathbf{A}$ la dimension de Krull de $\mathbf{A}_{\mathrm{K}}^{x}$ est $\leq \ell-1$.

3. Pour tout idéal de type fini $\mathfrak{j}$ de $\mathbf{A}$ la dimension de Krull de $\mathbf{A}_{\mathrm{K}}^{\mathfrak{j}}$ est $\leq \ell-1$.

4. Pour tout $x \in \mathbf{A}$ la dimension de Krull de $\mathbf{A}_{x}^{\mathrm{K}}$ est $\leq \ell-1$.

Ce théorème nous donne une bonne signification intuitive de la dimension de Krull.

Avec le fait 4.2 on obtient le même théorème en mathématiques classiques.

$\mathrm{Vu}$ son importance, nous allons donner des preuves directes simples des équivalences entre les points 1, 2 et 4 en mathématiques classiques.

Démonstration. - Preuve directe en mathématiques classiques Montrons d'abord l'équivalence des points 1 et 2 . Rappelons que les idéaux premiers de $S^{-1} \mathbf{A}$ sont de la forme $S^{-1} \mathfrak{p}$ où $\mathfrak{p}$ est un idéal premier de $\mathbf{A}$ qui ne coupe pas $S$. L'équivalence résulte alors clairement des deux affirmations suivantes.

(a) Soit $x \in \mathbf{A}$, si $\mathfrak{m}$ est un idéal maximal de $\mathbf{A}$ il coupe toujours $\mathrm{S}_{x}^{\mathrm{K}}$. En effet si $x \in \mathfrak{m}$ c'est clair et sinon, $x$ est inversible modulo $\mathfrak{m}$ ce qui signifie que $1+x \mathbf{A}$ coupe $\mathfrak{m}$.

(b) Si $\mathfrak{m}$ est un idéal maximal de $\mathbf{A}$, et si $x \in \mathfrak{m} \backslash \mathfrak{p}$ où $\mathfrak{p}$ est un idéal premier contenu dans $\mathfrak{m}$, alors $\mathfrak{p} \cap \mathrm{S}_{x}^{\mathrm{K}}=\emptyset$ : en effet si $x(1+x y) \in \mathfrak{p}$ alors, puisque $x \notin \mathfrak{p}$ on a $1+x y \in \mathfrak{p} \subseteq \mathfrak{m}$, ce qui donne la contradiction $1 \in \mathfrak{m}$ (puisque $x \in \mathfrak{m}$ ).

Ainsi, si $\mathfrak{p}_{0} \subsetneq \cdots \subsetneq \mathfrak{p}_{\ell}$ est une chaîne avec $\mathfrak{p}_{\ell}$ maximal, elle est raccourcie d'au moins son dernier terme lorsqu'on localise en $\mathrm{S}_{x}^{\mathrm{K}}$, et elle n'est raccourcie que de son dernier terme si $x \in \mathfrak{p}_{\ell} \backslash \mathfrak{p}_{\ell-1}$. L'équivalence des points 1 et 4 se démontre de manière «opposée », en remplaçant les idéaux premiers par les filtres premiers. On remarque d'abord que les filtres premiers de $\mathbf{A} / \mathfrak{J}$ sont de la forme $(S+\mathfrak{J}) / \mathfrak{J}$, où $S$ est un filtre premier de $\mathbf{A}$ qui ne coupe pas $\mathfrak{J}$. Il suffit alors de démontrer les deux affirmations «opposées » de (a) et (b) qui sont les suivantes :

(a') Soit $x \in \mathbf{A}$, si $S$ est un filtre maximal de $\mathbf{A}$ il coupe toujours $\mathrm{K}_{\mathbf{A}}^{x}$. En effet si $x \in S$ c'est clair et sinon, puisque $S$ est maximal $S x^{\mathbb{N}}$ contient 0 , ce qui signifie qu'il y a un entier $n$ et un élément $s$ de $S$ tels que $s x^{n}=0$. Alors $(s x)^{n}=0$ et $s \in(\sqrt{0}: x) \subseteq \mathrm{K}_{\mathbf{A}}^{x}$.

(b') Si $S$ est un filtre maximal de $\mathbf{A}$, et si $x \in S \backslash S^{\prime}$ où $S^{\prime} \subseteq S$ est un filtre premier, alors $S^{\prime} \cap \mathrm{K}_{\mathbf{A}}^{x}=\emptyset$. En effet si $a x+b \in S^{\prime}$ avec $(b x)^{n}=0$ alors, puisque $x \notin S^{\prime}$ on a $a x \notin S^{\prime}$ et, vu que $S^{\prime}$ est premier, $b \in S^{\prime} \subseteq S$, mais comme $x \in S,(b x)^{n}=0 \in S$ ce qui est absurde.

En outre le théorème 4.17 redonne la caractérisation constructive élémentaire de cette dimension en terme d'identités algébriques comme dans $[4,21]$ :

Corollaire 4.18. - Les propriétés suivantes sont équivalentes :

1. La dimension de Krull de $\mathbf{A}$ est $\leq \ell$. 
2. Pour tous $x_{0}, \ldots, x_{\ell} \in \mathbf{A}$ il existe $b_{0}, \ldots, b_{\ell} \in \mathbf{A}$ tels que

$$
\left.\begin{array}{rl}
\mathrm{D}_{\mathbf{A}}\left(b_{0} x_{0}\right) & =\mathrm{D}_{\mathbf{A}}(0) \\
\mathrm{D}_{\mathbf{A}}\left(b_{1} x_{1}\right) & \leq \mathrm{D}_{\mathbf{A}}\left(b_{0}, x_{0}\right) \\
\vdots & \vdots \\
\mathrm{D}_{\mathbf{A}}\left(b_{\ell} x_{\ell}\right) & \leq \mathrm{D}_{\mathbf{A}}\left(b_{\ell-1}, x_{\ell-1}\right) \\
\mathrm{D}_{\mathbf{A}}(1) & =\mathrm{D}_{\mathbf{A}}\left(b_{\ell}, x_{\ell}\right)
\end{array}\right\}
$$

3. Pour tous $x_{0}, \ldots, x_{\ell} \in \mathbf{A}$ il existe $a_{0}, \ldots, a_{\ell} \in \mathbf{A}$ et $m_{0}, \ldots, m_{\ell} \in \mathbb{N}$ tels que

$$
x_{0}^{m_{0}}\left(x_{1}^{m_{1}} \cdots\left(x_{\ell}^{m_{\ell}}\left(1+a_{\ell} x_{\ell}\right)+\cdots+a_{1} x_{1}\right)+a_{0} x_{0}\right)=0 .
$$

Démonstration. - Montrons l'equivalence de (1) et (3). Utilisons par exemple pour (1) la caractérisation via les localisés $\mathbf{A}_{x}^{\mathrm{K}}$. L'équivalence pour la dimension 0 est claire. Supposons la chose établie pour la dimension $\leq \ell$. On voit alors que $S^{-1} \mathbf{A}$ est de dimension $\leq \ell$ si et seulement si pour tous $x_{0}, \ldots, x_{\ell} \in \mathbf{A}$ il existe $a_{0}, \ldots, a_{\ell} \in \mathbf{A}, m_{0}, \ldots, m_{\ell} \in \mathbb{N}$ et $s \in S$ tels que

$$
x_{0}^{m_{0}}\left(x_{1}^{m_{1}} \cdots\left(x_{\ell}^{m_{\ell}}\left(s+a_{\ell} x_{\ell}\right)+\cdots+a_{1} x_{1}\right)+a_{0} x_{0}\right)=0 .
$$

Il reste donc à remplacer $s$ par un élément arbitraire de la forme $x_{\ell+1}^{m_{\ell+1}}\left(1+a_{\ell+1} x_{\ell+1}\right)$. On a $(3) \Rightarrow(2)$ en prenant $: b_{\ell}=1+a_{\ell} x_{\ell}$, et $b_{k-1}=x_{k}^{m_{k}} b_{k}+a_{k-1} x_{k-1}$, pour $k=\ell, \ldots, 1$. On a $(2) \Rightarrow(1)$ en considérant la caractérisation (4) de la dimension de Krull d'un treillis distributif donnée dans la proposition 3.10 et en l'appliquant au treillis de Zariski Zar A avec $S=$ $\left\{\mathrm{D}_{\mathbf{A}}(x) \mid x \in \mathbf{A}\right\}$. On pourrait aussi v'erifier par un calcul direct que $(2) \Rightarrow(3)$.

Remarque. - Le système d'inégalités (33) dans le point (2) du corollaire précédent établit une relation intéressante et symétrique entre les deux suites $\left(b_{0}, \ldots, b_{\ell}\right)$ et $\left(x_{0}, \ldots, x_{\ell}\right)$. Lorsque $\ell=0$, cela signifie $\mathrm{D}_{\mathbf{A}}\left(b_{0}\right) \wedge \mathrm{D}_{\mathbf{A}}\left(x_{0}\right)=0$ et $\mathrm{D}_{\mathbf{A}}\left(b_{0}\right) \vee \mathrm{D}_{\mathbf{A}}\left(x_{0}\right)=1$, c'est-à-dire que les deux éléments $\mathrm{D}_{\mathbf{A}}\left(b_{0}\right)$ et $\mathrm{D}_{\mathbf{A}}\left(x_{0}\right)$ sont compléments l'un de l'autre dans Zar A. Dans Spec A cela signifie que les ouverts de base correspondants sont complémentaires. Nous introduisons donc la terminologie suivante : lorsque deux suites $\left(b_{0}, \ldots, b_{\ell}\right)$ et $\left(x_{0}, \ldots, x_{\ell}\right)$ vérifient les inégalités $(33)$ nous dirons qu'elles sont complémentaires.

Remarque. - Il est facile d'établir constructivement que

$$
\operatorname{Kdim}\left(\mathbf{K}\left[X_{1}, \ldots, X_{n}\right]\right)=n
$$

lorsque $\mathbf{K}$ est un corps, ou même un anneau zéro dimensionnel (cf. [4]). On peut aussi traiter de façon constructive la dimension de Krull des anneaux géométriques (les $\mathbf{K}$-algèbres de présentation finie). Donc les théorèmes des sections 5 et suivantes ont un contenu algorithmique clair pour ces anneaux (comme pour tout anneau où l'on est capable d'expliciter constructivement la dimension de Krull).

Remarque. - On a aussi (déjà démontré pour les treillis distributifs) les résultats suivants :

- si $\mathbf{B}$ est un quotient ou un localisé de $\mathbf{A}$, alors $K \operatorname{dim} \mathbf{B} \leq \mathrm{Kdim} \mathbf{A}$,

- si $\left(\mathfrak{a}_{i}\right)_{1 \leq i \leq m}$ est une famille finie d'idéaux de $\mathbf{A}$ et $\mathfrak{a}=\bigcap_{i=1}^{m} \mathfrak{a}_{i}$, alors

$$
\operatorname{Kdim}(\mathbf{A} / \mathfrak{a})=\sup _{i} \operatorname{Kdim}\left(\mathbf{A} / \mathfrak{a}_{i}\right) .
$$

- si $\left(S_{i}\right)_{1 \leq i \leq m}$ une famille finie de monoïdes comaximaux de $\mathbf{A}$ alors $\operatorname{Kdim}(\mathbf{A})=\sup _{i} \operatorname{Kdim}\left(\mathbf{A}_{S_{i}}\right)$.

- en mathématiques classiques on $\operatorname{Kdim}(\mathbf{A})=\sup _{\mathfrak{m}} \operatorname{Kdim}\left(\mathbf{A}_{\mathfrak{m}}\right)$, où $\mathfrak{m}$ partcourt tous les idéaux maximaux.

Remarque. - On peut illustrer le corollaire 4.18 ci-dessus en introduisant « l'idéal bord de Krull itéré ». Pour $x_{1}, \ldots, x_{n} \in \mathbf{A}$ considérons $\left(\mathbf{A}_{\mathrm{K}}^{x_{1}}\right)_{\mathrm{K}}^{x_{2}},\left(\left(\mathbf{A}_{\mathrm{K}}^{x_{1}}\right)_{\mathrm{K}}^{x_{2}}\right)_{\mathrm{K}}^{x_{3}}$, etc. . les anneaux bords supérieurs successifs, et notons $\mathrm{K}_{\mathbf{A}}\left[x_{1}, \ldots, x_{\ell}\right]$ le noyau de la projection canonique $\mathbf{A} \rightarrow\left(\ldots\left(\mathbf{A}_{\mathrm{K}}^{x_{1}}\right)^{\cdots}\right)_{\mathrm{K}}^{x_{\ell}}$. Alors on a $y \in \mathrm{K}_{\mathbf{A}}\left[x_{0}, \ldots, x_{\ell}\right]$ si et seulement si $\exists a_{0}, \ldots, a_{\ell} \in \mathbf{T}$ et $m_{0}, \ldots, m_{\ell} \in \mathbb{N}$ vérifiant :

$$
x_{0}^{m_{0}}\left(x_{1}^{m_{1}} \cdots\left(x_{\ell}^{m_{\ell}}\left(y+a_{\ell} x_{\ell}\right)+\cdots+a_{1} x_{1}\right)+a_{0} x_{0}\right)=0 .
$$

Et la dimension de Krull est $\leq \ell$ si et seulement si pour tous $x_{0}, \ldots, x_{\ell} \in \mathbf{A}$ on a $1 \in \mathrm{K}_{\mathbf{A}}\left[x_{0}, \ldots, x_{\ell}\right]$. 


\subsection{Dimensions de Heitmann. -}

Le spectre de Heitmann. - L'espace spectral que Heitmann a défini pour remplacer le jspectrum, c'est-à-dire l'adhérence pour la topologie constructible du spectre maximal dans Spec A, correspond à la définition suivante.

Définition 4.19. - On appelle spectre de Heitmann d'un anneau commutatif A le sous espace Jspec(Zar A) de Spec A. On le note aussi Jspec A. On note jspec A pour jspec(Zar A), c'est-à-dire le j-spectrum de l'anneau au sens usuel.

En mathématiques classiques le théorème 2.11 donne :

Fait 4.20. - Pour tout anneau commutatif $\mathbf{A}$, le spectre de Heitmann de $\mathbf{A}$ s'identifie à l'espace spectral Spec(Heit A) (au sens des treillis distributifs).

On a alors la définition constructive élémentaire sans points de la dimension introduite par Heitmann.

Définition 4.21. - La J-dimension de Heitmann de A, notée Jdim A, est la dimension de Krull de Heit(A), autrement dit c'est la Jdim de Zar A.

En mathématiques classiques Jdim A est égal à la dimension de l'espace spectral Jspec A, définie de manière abstraite « avec points ».

On peut aussi noter jdim A pour la dimension de jspec A, qui n'est pas un espace spectral (et nous ne proposons pas de définition constructive sans point pour cette dimension).

Remarque. - Précisons la signification de $\operatorname{Jdim} \mathbf{A} \leq \ell$ dans le cas des anneaux commutatifs. Comme il s'agit de la dimension de Krull de Heit $\mathbf{A}$ et que les éléments de Heit A s'identifient aux radicaux de Jacobson d'idéaux de type fini on obtient la caractérisation suivante :

$\forall x_{0}, \ldots, x_{\ell} \in \mathbf{A} \exists \mathfrak{a}_{0}, \ldots, \mathfrak{a}_{\ell}$, idéaux de type fini de $\mathbf{A}$ tels que :

$$
\begin{array}{rlc}
x_{0} \mathfrak{a}_{0} & \subseteq & \mathrm{J}_{\mathbf{A}}(0) \\
x_{1} \mathfrak{a}_{1} & \subseteq & \mathrm{J}_{\mathbf{A}}\left(\left\langle x_{0}\right\rangle+\mathfrak{a}_{0}\right) \\
\vdots & \vdots & \vdots \\
x_{\ell} \mathfrak{a}_{\ell} & \subseteq & \mathrm{J}_{\mathbf{A}}\left(\left\langle x_{\ell-1}\right\rangle+\mathfrak{a}_{\ell-1}\right) \\
\langle 1\rangle & = & \mathrm{J}_{\mathbf{A}}\left(\left\langle x_{\ell}\right\rangle+\mathfrak{a}_{\ell}\right)
\end{array}
$$

On ne peut apparemment pas éviter le recours aux idéaux de type fini et cela fait que l'on n'obtient pas une définition « au premier ordre».

Notez que chaque appartenance $x \in \mathrm{J}_{\mathbf{A}}\left(y_{1}, \ldots, y_{m}\right)$ s'exprime elle-même par $: \forall z \in \mathbf{A}, 1+x z$ est inversible modulo $\left\langle y_{1}, \ldots, y_{m}\right\rangle$, c'est-à-dire encore

$$
\forall z \in \mathbf{A} \exists t, u_{1}, \ldots, u_{m} \in \mathbf{A}, \quad 1=(1+x z) t+u_{1} y_{1}+\cdots+u_{m} y_{m} .
$$

\section{Dimension et bord de Heitmann. -}

Définition 4.22. - La dimension de Heitmann d'un anneau commutatif est la dimension de Heitmann de son treillis de Zariski.

Définition 4.23. - Soit $\mathbf{A}$ un anneau commutatif, $x \in \mathbf{A}$ et $\mathbf{j}$ un idéal de type fini. Le bord de Heitmann de $\mathbf{j}$ dans $\mathbf{A}$ est l'anneau quotient $\mathbf{A} / \mathrm{H}_{\mathbf{A}}(\mathfrak{j})$ avec

$$
\mathrm{H}_{\mathbf{A}}(\mathfrak{j}):=\mathfrak{j}+\left(\mathrm{J}_{\mathbf{A}}(0): \mathfrak{j}\right)
$$

qui est aussi appelé l'idéal bord de Heitmann de j dans $\mathbf{A}$. On notera aussi

$$
\mathrm{H}_{\mathbf{A}}\left(y_{1}, \ldots, y_{n}\right) \text { pour } \mathrm{H}_{\mathbf{A}}\left(\left\langle y_{1}, \ldots, y_{n}\right\rangle\right), \mathrm{H}_{\mathbf{A}}^{x} \text { pour } \mathrm{H}_{\mathbf{A}}(x) \text { et } \mathbf{A}_{\mathrm{H}}^{x} \text { pour } \mathbf{A} / \mathrm{H}_{\mathbf{A}}^{x} \text {. }
$$

Ainsi un élément arbitraire de $\mathrm{H}_{\mathbf{A}}\left(y_{1}, \ldots, y_{n}\right)$ s'écrit $\sum_{i} a_{i} y_{i}+b$ avec tous les $b y_{i}$ dans $\mathrm{J}_{\mathbf{A}}(0)$. La proposition suivante résulte des bonnes propriétés de la correspondance bijective $\mathcal{I Z}_{\mathbf{A}}$ (voir les faits $4.2,4.3,4.4$ et 4.12 ). 
Proposition 4.24. - Pour un idéal de type fini $\mathfrak{j}$ le bord de Heitmann de $\mathbf{j}$ au sens des anneaux commutatifs et celui au sens des treillis distributifs se correspondent. Plus précisément, avec $j=$ $\mathrm{D}_{\mathbf{A}}(\mathfrak{j})$ et $\mathbf{T}=\operatorname{Zar} \mathbf{A}$, on $a$ :

$$
\mathcal{I Z}_{\mathbf{A}}\left(\mathrm{H}_{\mathbf{A}}(\mathfrak{j})\right)=\mathrm{H}_{\mathbf{T}}(j) \text {, et } \operatorname{Zar}\left(\mathbf{A} / \mathrm{H}_{\mathbf{A}}(\mathfrak{j})\right) \simeq \mathbf{T} /\left(\mathrm{H}_{\mathbf{T}}(j)=0\right)=\mathbf{T}_{\mathbf{H}}^{j} .
$$

Comme corollaire des propositions 3.25 et 4.24 on obtient.

Proposition 4.25. - Pour un anneau commutatif $\mathbf{A}$ et un entier $\ell \geq 0$ les propriétés suivantes sont équivalentes :

1. La dimension de Heitmann de $\mathbf{A}$ est $\leq \ell$.

2. Pour tout $x \in \mathbf{A}, \operatorname{Hdim}\left(\mathbf{A} / \mathrm{H}_{\mathbf{A}}(x)\right) \leq \ell-1$.

3. Pour tout idéal de type fini $\mathbf{j}$ de $\mathbf{A}, \operatorname{Hdim}\left(\mathbf{A} / \mathrm{H}_{\mathbf{A}}(\mathfrak{j})\right) \leq \ell-1$.

Remarque. - La dimension de Heitmann de $\mathbf{A}$ peut donc être définie de manière inductive comme suit :

$-\operatorname{Hdim} \mathbf{A}=-1$ si et seulement si $1_{\mathbf{A}}=0_{\mathbf{A}}$.

- Pour $\ell \geq 0, \operatorname{Hdim} \mathbf{A} \leq \ell$ si et seulement si pour tout $x \in \mathbf{A}, \operatorname{Hdim}\left(\mathbf{A} / \mathrm{H}_{\mathbf{A}}(x)\right) \leq \ell-1$.

On peut illustrer cette définition. Nous introduisons «l'idéal bord de Heitmann itéré ». Pour $x_{1}, \ldots, x_{n} \in \mathbf{A}$ nous notons

$$
\mathbf{A}_{\mathrm{H}}\left[x_{1}\right]=\mathbf{A}_{\mathrm{H}}^{x_{1}}, \mathbf{A}_{\mathrm{H}}\left[x_{1}, x_{2}\right]=\left(\mathbf{A}_{\mathrm{H}}^{x_{1}}\right)_{\mathrm{H}}^{x_{2}}, \mathbf{A}_{\mathrm{H}}\left[x_{1}, x_{2}, x_{3}\right]=\left(\left(\mathbf{A}_{\mathrm{H}}^{x_{1}}\right)_{\mathrm{H}}^{x_{2}}\right)_{\mathrm{H}}^{x_{3}}, \text { etc } \ldots
$$

les anneaux bords de Heitmann successifs, et $\mathrm{H}\left[\mathbf{A} ; x_{1}, \ldots, x_{k}\right]=\mathrm{H}_{\mathbf{A}}\left[x_{1}, \ldots, x_{k}\right]$ désigne le noyau de la projection canonique $\mathbf{A} \rightarrow \mathbf{A}_{\mathrm{H}}\left[x_{1}, \ldots, x_{k}\right]$. Pour décrire ces idéaux nous avons besoin de la notation

$$
\lceil z, x, a, y, b\rceil=1+(1+(z+a x) x y) b .
$$

Alors on a :

$-z \in \mathrm{H}_{\mathbf{A}}\left[x_{0}\right]$ si et seulement si :

$$
\exists a_{0} \forall y_{0} \exists b_{0},\left\lceil z, x_{0}, a_{0}, y_{0}, b_{0}\right\rceil=0
$$

- $z \in \mathrm{H}_{\mathbf{A}}\left[x_{0}, x_{1}\right]$ si et seulement si :

$$
\exists a_{1} \forall y_{1} \exists b_{1} \exists a_{0} \forall y_{0} \exists b_{0},\left\lceil\left\lceil z, x_{1}, a_{1}, y_{1}, b_{1}\right\rceil, x_{0}, a_{0}, y_{0}, b_{0}\right\rceil=0
$$

$-z \in \mathrm{H}_{\mathbf{A}}\left[x_{0}, x_{1}, x_{2}\right]$ si et seulement si :

$\exists a_{2} \forall y_{2} \exists b_{2} \exists a_{1} \forall y_{1} \exists b_{1} \exists a_{0} \forall y_{0} \exists b_{0},\left\lceil\left\lceil\left\lceil z, x_{2}, a_{2}, y_{2}, b_{2}\right\rceil, x_{1}, a_{1}, y_{1}, b_{1}\right\rceil, x_{0}, a_{0}, y_{0}, b_{0}\right\rceil=0$

Et ainsi de suite. Et la dimension de Heitmann est $\leq \ell$ si et seulement si pour tous $x_{0}, \ldots, x_{\ell} \in \mathbf{A}$ on a $1 \in \mathrm{H}_{\mathbf{A}}\left[x_{0}, \ldots, x_{\ell}\right]$.

Proposition 4.26. - Soit $\mathfrak{j}=\left\langle j_{1}, \ldots, j_{n}\right\rangle$ un idéal de type fini. Notons $\varphi=\mathrm{J}_{\mathbf{A}}(\mathfrak{j})=\mathrm{J}_{\mathbf{A}}\left(j_{1}\right) \vee$ $\cdots \vee \mathrm{J}_{\mathbf{A}}\left(j_{n}\right)$. Alors Heit $\left(\mathbf{A} / \mathrm{H}_{\mathbf{A}}(\mathfrak{j})\right)$ s'identifie naturellement avec un quotient de (Heit $\left.\mathbf{A}\right)_{\mathrm{K}}^{\varphi}$. Il y a égalité lorsque Heit $\mathbf{A}$ est une algèbre de Heyting, donc en particulier lorsque Jspec $\mathbf{A}$ est nœethérien.

Démonstration. - Déjà démontré pour un treillis distributif arbitraire à la place de Zar A (proposition 3.17).

Remarque. - On a aussi (déjà démontré pour les treillis distributifs) les résultats suivants :

- on a toujours $\operatorname{Hdim} \mathbf{A} \leq \operatorname{Jdim} \mathbf{A} \leq \operatorname{Kdim}\left(\mathbf{A} / \mathrm{J}_{\mathbf{A}}(0)\right)$,

- si $\left(\mathfrak{a}_{i}\right)_{1 \leq i \leq m}$ est une famille finie d'idéaux de $\mathbf{A}$ et $\mathfrak{a}=\bigcap_{i=1}^{m} \mathfrak{a}_{i}$, alors $\operatorname{Hdim}(\mathbf{A} / \mathfrak{a})=\sup _{i} \operatorname{Hdim}\left(\mathbf{A} / \mathfrak{a}_{i}\right)$.

- si Heit $\mathbf{A}$ est une algèbre de Heyting (en particulier si Jspec $\mathbf{A}$ est nothérien) on a $\operatorname{Hdim} \mathbf{A}=$ $\operatorname{Jdim} \mathbf{A}$,

- si jspec $\mathbf{A}$ est nothérien, alors jspec $\mathbf{A}=\operatorname{Jspec} \mathbf{A}$ et $\operatorname{Hdim} \mathbf{A}=\operatorname{Jdim} \mathbf{A}=\operatorname{jdim} \mathbf{A}$.

$-\operatorname{Hdim} \mathbf{A} \leq 0 \Leftrightarrow \operatorname{Jdim} \mathbf{A} \leq 0 \Leftrightarrow \operatorname{Kdim}\left(\mathbf{A} / \mathrm{J}_{\mathbf{A}}(0)\right) \leq 0$. 
Notez que le treillis Heit $\mathbf{A}=\mathbf{T}$ est une algèbre de Heyting si et seulement si est vérifiée la propriété suivante :

$$
\begin{array}{r}
\forall \mathfrak{a}, \mathfrak{b} \in \mathbf{T} \exists \mathfrak{c} \in \mathbf{T}(\mathfrak{c} \mathfrak{b} \subseteq \mathfrak{a} \text { et } \forall x \in \mathbf{A}(x \mathfrak{b} \subseteq \mathfrak{a} \Rightarrow x \in \mathfrak{c})) \\
\left(\mathfrak{a}=\mathrm{J}_{\mathbf{A}}\left(a_{1}, \ldots, a_{n}\right), \mathfrak{b}=\mathrm{J}_{\mathbf{A}}\left(b_{1}, \ldots, b_{m}\right), \mathfrak{c}=\mathrm{J}_{\mathbf{A}}\left(c_{1}, \ldots, c_{\ell}\right)\right) .
\end{array}
$$

\section{Le théorème de Kronecker et le stable range de Bass (versions non nœthériennes de Heitmann)}

Dans cette section et celles qui suivent on établit la version élémentaire, non nœthérienne et constructive des théorèmes cités dans les titres. La version classique de ces théorèmes est pour l'essentiel due à Heitmann [15], hormis pour le théorème de Swan et le « Bass cancellation theorem » traités dans la section 7 .

Pour le théorème de Kronecker en particulier seule la dimension de Krull intervient, et le théorème se trouve donc dans [15] (voir aussi [14]).

Pour les autres théorèmes des sections 5 et 6 , notre «dimension de Heitmann » peut être dans certains cas strictement plus petite que la dimension utilisée par Heitmann (celle de son J-spectrum), et donc notre version est dans ce cas « meilleure ».

Enfin concernant le théorème de Swan et toute la section 7. Nos versions sont constructives et non nothériennes, et la « dimension de Heitmann » que nous utilisons coïncide dans le cas où jspec A est nœethérien avec celle utilisée par Swan, ce qui n'est pas le cas de la $\delta$-dim utilisée par Heitmann.

Les preuves données dans les sections suivantes sont essentiellement celles de [3] (pour le théorème de Kronecker) et de [5], quelquefois améliorées en tenant compte de [9]. Nous avons rajouté en outre quelques précisions, ainsi que les résultats de la section 5.3.

5.1. Le théorème de Kronecker. - Ce théorème a d'abord été démontré par Kronecker sous la forme suivante : une variété algébrique dans $\mathbb{C}^{n}$ peut toujours être définie par $n+1$ équations.

Il a été étendu au cas des anneaux nothériens (par van der Waerden, dans [25]) sous la forme suivante : dans un anneau nothérien de dimension de Krull $n$, tout idéal a même radical qu'un idéal engendré par au plus $n+1$ éléments.

La version de Kronecker a été améliorée par divers auteurs dans $[\mathbf{1 1}, \mathbf{2 3}]$ qui ont montré que $n$ équations suffisent en général. On peut trouver une preuve constructive de ce théorème dans [7]. Par ailleurs on ne sait toujours pas si toute courbe dans l'espace complexe de dimension 3 est ou non intersection de deux surfaces.

Heitmann [15] a enfin généralisé la version van der Waerden au cas non nothérien.

Le lemme suivant, bien que terriblement anodin, est une clef essentielle.

Lemme 5.1. - Pour $u, v \in \mathbf{A}$ on a

$$
\begin{aligned}
\mathrm{D}_{\mathbf{A}}(u, v) & =\mathrm{D}_{\mathbf{A}}(u+v, u v)=\mathrm{D}_{\mathbf{A}}(u+v) \vee \mathrm{D}_{\mathbf{A}}(u v) \text { et donc aussi } \\
\mathrm{J}_{\mathbf{A}}(u, v) & =\mathrm{J}_{\mathbf{A}}(u+v, u v)=\mathrm{J}_{\mathbf{A}}(u+v) \vee \mathrm{J}_{\mathbf{A}}(u v)
\end{aligned}
$$

En particulier

$-S i u v \in \mathrm{D}_{\mathbf{A}}(0)$, alors $\mathrm{D}_{\mathbf{A}}(u, v)=\mathrm{D}_{\mathbf{A}}(u+v)$

- Si uv $\in \mathrm{J}_{\mathbf{A}}(0)$, alors $\mathrm{J}_{\mathbf{A}}(u, v)=\mathrm{J}_{\mathbf{A}}(u+v)$

Rappelons que deux suites qui vérifient les inégalités (33) dans le corollaire 4.18 sont dites complémentaires.

Lemme 5.2. - Si $\left(b_{1}, \ldots, b_{n}\right)$ et $\left(x_{1} \ldots, x_{n}\right)$ sont deux suites complémentaires dans $\mathbf{A}$ alors pour tout $a \in \mathbf{A}$ on $a$ :

$$
\mathrm{D}_{\mathbf{A}}\left(a, b_{1}, \ldots, b_{n}\right)=\mathrm{D}_{\mathbf{A}}\left(b_{1}+a x_{1}, \ldots, b_{n}+a x_{n}\right),
$$

c'est-à-dire encore : $a \in \mathrm{D}_{\mathbf{A}}\left(b_{1}+a x_{1}, \ldots, b_{n}+a x_{n}\right)$. 
Démonstration. - Pour $n=0$ l'anneau est trivial et $\mathrm{D}_{\mathbf{A}}(a)=\mathrm{D}_{\mathbf{A}}(\emptyset)$.

Faisons aussi le cas $n=1$ bien que ce soit inutile (vue la preuve par récurrence). On a $b_{1} x_{1} \in \mathrm{D}_{\mathbf{A}}(0)$ et $1 \in \mathrm{D}_{\mathbf{A}}\left(b_{1}, x_{1}\right)$. A fortiori $b_{1} a x_{1} \in \mathrm{D}_{\mathbf{A}}(0)$ et $a \in \mathrm{D}_{\mathbf{A}}\left(b_{1}, a x_{1}\right)$. Et le lemme 5.1 nous dit que $\mathrm{D}_{\mathbf{A}}\left(b_{1}, a x_{1}\right)=\mathrm{D}_{\mathbf{A}}\left(b_{1}+a x_{1}\right)$.

Donnons maintenant la preuve de la récurrence. On considère l'anneau quotient $\mathbf{B}=\mathbf{A} / \mathrm{D}_{\mathbf{A}}\left(b_{1}, x_{1}\right)$. Par hypothèse de récurrence on a pour tout $a$,

$$
a \in \mathrm{D}_{\mathbf{B}}\left(b_{2}+a x_{2}, \ldots, b_{n}+a x_{n}\right) .
$$

Cela signifie dans A

$$
a \in \mathrm{D}_{\mathbf{A}}\left(b_{1}, x_{1}, b_{2}+a x_{2}, \ldots, b_{n}+a x_{n}\right) .
$$

On en déduit $a \in \mathrm{D}_{\mathbf{A}}\left(b_{1}, a x_{1}, b_{2}+a x_{2}, \ldots, b_{n}+a x_{n}\right)\left(\operatorname{car} a \in \mathrm{D}_{\mathbf{A}}(\mathfrak{J})\right.$ implique $\left.a \in \mathrm{D}_{\mathbf{A}}(a \mathfrak{J})\right)$. Mais $\mathrm{D}_{\mathbf{A}}\left(b_{1} x_{1}\right)=0$ implique $\mathrm{D}_{\mathbf{A}}\left(b_{1} a x_{1}\right)=0$ et le lemme 5.1 nous dit que $\mathrm{D}_{\mathbf{A}}\left(b_{1}, a x_{1}\right)=\mathrm{D}_{\mathbf{A}}\left(b_{1}+\right.$ $\left.a x_{1}\right)$.

Théorème 5.3. - (de Kronecker, avec la dimension de Krull, sans noethérianité)

1. Soit $n \geq 0$. Si $\mathrm{Kdim} \mathbf{A}<n$ et $b_{1}, \ldots, b_{n} \in \mathbf{A}$ alors il existe $x_{1}, \ldots, x_{n}$ tels que pour tout $a \in \mathbf{A} \mathrm{D}_{\mathbf{A}}\left(a, b_{1}, \ldots, b_{n}\right)=\mathrm{D}_{\mathbf{A}}\left(b_{1}+a x_{1}, \ldots, b_{n}+a x_{n}\right)$.

2. En conséquence, dans un anneau de dimension de Krull $\leq n$, tout idéal de type fini a même radical qu'un idéal engendré par au plus $n+1$ éléments.

Démonstration. - Le premier point est clair d'après le lemme 5.2 et le corollaire 4.18. La deuxième affirmation en découle car il suffit d'itérer le processus. En fait, si $\mathfrak{J}=\mathrm{D}_{\mathbf{A}}\left(b_{1}, \ldots, b_{n+r}\right)$ avec $\mathrm{Kdim} \mathbf{A} \leq n$ et $r \geq 2$ on obtient en fin de compte $\mathfrak{J}=\mathrm{D}_{\mathbf{A}}\left(b_{1}+c_{1}, \ldots, b_{n+1}+c_{n+1}\right)$ avec les $c_{i} \in\left\langle b_{n+2}, \ldots, b_{n+r}\right\rangle$.

5.2. Le théorème «stable range» de Bass. — Le théorème suivant est dû à Bass dans le cas nœthérien avec la dimension de Krull. La version non nœthérienne avec la Jdim est due à Heitmann. Nous donnons ici la version non noethérienne avec la Hdim, a priori plus générale.

Notez que la version non nœthérienne avec la dimension de Krull résulte directement du premier point dans le théorème 5.3 .

Théorème 5.4. - (de Bass, avec la dimension de Heitmann)

Soit $n \geq 0$. Si $\mathbf{H} \operatorname{dim} \mathbf{A}<n$ et $1 \in\left\langle a, b_{1}, \ldots, b_{n}\right\rangle$ alors il existe $x_{1}, \ldots, x_{n}$ tels que

$$
1 \in\left\langle b_{1}+a x_{1}, \ldots, b_{n}+a x_{n}\right\rangle .
$$

Démonstration. - On remarque que pour toute liste $L$

$$
1 \in\langle L\rangle \Longleftrightarrow 1 \in \mathrm{D}_{\mathbf{A}}(L) \Longleftrightarrow 1 \in \mathrm{J}_{\mathbf{A}}(L) .
$$

La preuve qui suit peut être considérée comme une répétition de celle du théorème 5.3 lorsque $a=1$ en remplaçant le bord de Krull par le bord de Heitmann. La preuve est par récurrence sur $n$. Lorsque $n=0$ l'anneau est trivial et $\mathrm{D}_{\mathbf{A}}(1)=\mathrm{D}_{\mathbf{A}}(\emptyset)$.

Supposons $n \geq 1$. Soit $\mathfrak{j}=\mathrm{H}_{\mathbf{A}}\left(b_{n}\right)$ l'idéal bord de Heitmann de $b_{n}$. Puisque $\mathbf{A} / \mathbf{j}$ est de dimension de Heitmann $\leq n-2$ l'hypothèse de récurrence nous donne $x_{1}, \ldots, x_{n-1} \in \mathbf{A}$ tels que

$$
1 \in \mathrm{D}\left(b_{1}+x_{1} a, \ldots, b_{n-1}+x_{n-1} a\right) \text { dans } \mathbf{A} / \mathfrak{j} .
$$

Notons $L$ pour $b_{1}+x_{1} a, \ldots, b_{n-1}+x_{n-1} a$. Par définition du bord de Heitmann, l'égalité ci-dessus implique qu'il existe $x_{n}$ tel que

$$
x_{n} b_{n} \in \mathrm{J}_{\mathbf{A}}(0) \quad \text { et } \quad 1 \in \mathrm{D}_{\mathbf{A}}\left(L, b_{n}, x_{n}\right)=\mathrm{D}_{\mathbf{A}}\left(L, b_{n}\right) \vee \mathrm{D}_{\mathbf{A}}\left(x_{n}\right)
$$

Puisque

$$
1 \in \mathrm{D}_{\mathbf{A}}\left(L, b_{n}, a\right)=\mathrm{D}_{\mathbf{A}}\left(L, b_{n}\right) \vee \mathrm{D}_{\mathbf{A}}(a)
$$

cela implique par distributivité

$$
1 \in \mathrm{D}_{\mathbf{A}}\left(L, b_{n}, x_{n} a\right)=\mathrm{J}_{\mathbf{A}}\left(L, b_{n}, x_{n} a\right)
$$


Puisque $b_{n} x_{n} a \in \mathrm{J}_{\mathbf{A}}(0)$ le lemme 5.1 nous dit que $\mathrm{J}_{\mathbf{A}}\left(b_{n}, x_{n} a\right)=\mathrm{J}_{\mathbf{A}}\left(b_{n}+x_{n} a\right)$, et donc que

$$
\mathrm{J}_{\mathbf{A}}\left(L, b_{n}+x_{n} a\right)=\mathrm{J}_{\mathbf{A}}\left(L, b_{n}, x_{n} a\right)=\langle 1\rangle,
$$

ce qui était le but recherché.

Rappelons qu'un vecteur $L$ de $\mathbf{A}^{m}$ est dit unimodulaire lorsque ses coordonnées engendrent l'idéal $\langle 1\rangle$, c'est-à-dire encore lorsque $\mathrm{D}_{\mathbf{A}}(L)=1$.

Corollaire 5.5. - Soit $n \geq 0$. Si $\operatorname{Hdim} \mathbf{A} \leq n$ et $V \in \mathbf{A}^{n+2}$ est unimodulaire, il peut être transformé en le vecteur $(1,0 \ldots, 0)$ par des manipulations élémentaires.

Il s'ensuit alors directement qu'un module stablement libre de rang $\geq n+1$ sur un anneau dont la dimension de Heitmann est $\leq n$ est libre (comme dans [20], p. 28), sans aucune hypothèse nœethérienne.

5.3. Une généralisation de Heitmann. - On traite ici constructivement le corollaire 2.2 de Heitmann [15], qui généralise à la fois les résultats de Bass et Kronecker. Cela débouche sur une amélioration du théorème de Kronecker. Dans [15] ces résultats sont établis pour la Jdim.

Lemme 5.6. - Supposons $b \in \mathrm{J}\left(\mathbf{A}\left[a^{-1}\right], 0\right)$.

1. Supposons $b \in \mathrm{J}\left(\mathbf{A}\left[a^{-1}\right], 0\right)$. Alors $a \in \mathrm{D}_{\mathbf{A}}\left(b_{1}, \ldots, b_{n}, b\right) \Rightarrow a \in \mathrm{D}_{\mathbf{A}}\left(b_{1}, \ldots, b_{n}\right)$.

2. Supposons que by $\in \mathrm{J}\left(\mathbf{A}\left[a^{-1}\right], 0\right)$ et $a \in \mathrm{D}_{\mathbf{A}}\left(b_{1}, \ldots, b_{n}, b, y\right)$. Alors :

$-a \in \mathrm{D}_{\mathbf{A}}\left(b_{1}, \ldots, b_{n}, b+y\right)$.

$-\mathrm{D}_{\mathbf{A}}\left(b_{1}, \ldots, b_{n}, b, y\right)=\mathrm{D}_{\mathbf{A}}\left(b_{1}, \ldots, b_{n}, b+a y\right)$.

Démonstration. - Montrons le point 1. Dire que $b$ est dans le radical de Jacobson de $\mathbf{A}\left[a^{-1}\right]$ signifie que pour tout $x \in \mathbf{A}$ et tout $p \in \mathbb{N}, 1+x b / a^{p}$ est inversible dans $\mathbf{A}\left[a^{-1}\right]$, c'est-à-dire $a \in \mathrm{D}_{\mathbf{A}}\left(a^{p}+x b\right)$. Or lorsqu'on dit $a \in \mathrm{D}_{\mathbf{A}}\left(b_{1}, \ldots, b_{n}, b\right)$ on affirme qu'il existe $x \in \mathbf{A}$ et $p \in \mathbb{N}$ tels que $a^{p}+x b \in \mathrm{D}_{\mathbf{A}}\left(b_{1}, \ldots, b_{n}\right)$, c'est-à-dire $\mathrm{D}_{\mathbf{A}}\left(a^{p}+x b\right) \leq \mathrm{D}_{\mathbf{A}}\left(b_{1}, \ldots, b_{n}\right)$.

Le premier item du point 2 résulte du point 1 et du lemme 5.1. Pour le deuxième item du point 2 , il suffit de montrer que $a \in \mathrm{D}_{\mathbf{A}}\left(b_{1}, \ldots, b_{n}, b+a y\right)$. Mais cela résulte du premier item, puisque $a \in \mathrm{D}_{\mathbf{A}}\left(b_{1}, \ldots, b_{n}, b, y\right)$ implique $a \in \mathrm{D}_{\mathbf{A}}\left(b_{1}, \ldots, b_{n}, b, a y\right)$.

Remarque. - On peut se demander si l'idéal $\mathrm{J}\left(\mathbf{A}\left[a^{-1}\right], 0\right)$ est le meilleur possible. La réponse est oui. L'implication du point 1 est vérifiée (pour tous $\left.b_{1}, \ldots, b_{n}\right)$ en remplaçant $J\left(\mathbf{A}\left[a^{-1}\right], 0\right)$ par $\mathfrak{J}$ seulement si $\mathfrak{J} \subseteq \mathrm{J}\left(\mathbf{A}\left[a^{-1}\right], 0\right)$.

Voici le corollaire 2.2 de Heitmann [15], avec la Hdim qui remplace la Jdim. Si $L=\left(b_{1}, \ldots, b_{n}\right) \in$ $\mathbf{A}^{n}$ nous écrivons $\mathrm{D}_{\mathbf{A}}(L)$ pour $\mathrm{D}_{\mathbf{A}}\left(b_{1}, \ldots, b_{n}\right)$.

Lemme 5.7. - Si $\operatorname{Hdim}\left(\mathbf{A}\left[a^{-1}\right]\right)<n, L \in \mathbf{A}^{n}$ et $\mathrm{D}_{\mathbf{A}}(b) \leq \mathrm{D}_{\mathbf{A}}(a) \leq \mathrm{D}_{\mathbf{A}}(b, L)$ alors il existe $X \in \mathbf{A}^{n}$ tel que $\mathrm{D}_{\mathbf{A}}(L+a X)=\mathrm{D}_{\mathbf{A}}(b, L)$. En outre nous pouvons prendre $X=$ a $Y$ avec $Y \in \mathbf{A}^{n}$.

Démonstration. - On raisonne par récurrence sur $n$, le cas $n=0$ étant trivial. On commence par chercher $X \in \mathbf{A}^{n}$. Soit $\mathfrak{j}=\mathrm{H}\left(\mathbf{A}\left[a^{-1}\right], b_{n}\right)$. On pose $\mathfrak{i}=\mathfrak{j} \cap \mathbf{A}$ et $\mathbf{A}^{\prime}=\mathbf{A} / \mathfrak{i}$. On obtient $\mathbf{A}\left[a^{-1}\right] / \mathfrak{j}=\mathbf{A}^{\prime}\left[a^{-1}\right]$ (en fait $\mathfrak{j} \cap \mathbf{A}$ est mis pour « l'image réciproque de $\mathfrak{j}$ dans $\mathbf{A}$ » et l'égalité est un isomorphisme canonique). Dans $\mathbf{A}^{\prime}$ on a $b_{n}=0$, de plus $\operatorname{Hdim}\left(\mathbf{A}^{\prime}\left[a^{-1}\right]\right)<n-1$, donc on peut appliquer l'hypothèse de récurrence avec $\mathbf{A}^{\prime}$ et $a, b, b_{1}, \ldots, b_{n-1}$, et on obtient $x_{1}, \ldots, x_{n-1} \in \mathbf{A}$ tels que $\mathrm{D}\left(b_{1}+a x_{1}, \ldots, b_{n-1}+a x_{n-1}\right)=\mathrm{D}\left(b, b_{1}, \ldots, b_{n-1}\right)$ dans $\mathbf{A}^{\prime}$. Cela signifie que

$$
a \in \mathrm{D}\left(b_{1}+x_{1} a, \ldots, b_{n-1}+x_{n-1} a\right) \text { dans } \mathbf{A}^{\prime} .
$$

Notons $L$ pour $b_{1}+x_{1} a, \ldots, b_{n-1}+x_{n-1} a$. Par définition du bord, l'égalité ci-dessus veut dire qu'il existe $x_{n}$ tel que $x_{n} b_{n} \in \mathrm{J}\left(\mathbf{A}\left[a^{-1}\right], 0\right)$ et $a \in \mathrm{D}_{\mathbf{A}}\left(L, b_{n}, x_{n}\right)$. On termine en utilisant le lemme $5.6(2)$. 
Finalement, nous pouvons appliquer le résultat avec $a^{2}$ à la place de $a$ puisque $\mathbf{A}\left[a^{-2}\right]=\mathbf{A}\left[a^{-1}\right]$ et $\mathrm{D}_{\mathbf{A}}(a)=\mathrm{D}_{\mathbf{A}}\left(a^{2}\right)$.

Pour $a \in \mathbf{A}$ on a toujours $\operatorname{Hdim}\left(\mathbf{A}\left[a^{-1}\right]\right) \leq \operatorname{Kdim}\left(\mathbf{A}\left[a^{-1}\right]\right) \leq \operatorname{Kdim}(\mathbf{A})$. Par conséquent le théorème suivant améliore le théorème de Kronecker.

Théorème 5.8. - (de Kronecker, avec la dimension de Heitmann, sans nœethérianité)

1. Soit $n \geq 0$. Si $\operatorname{Hdim}\left(\mathbf{A}\left[a^{-1}\right]\right)<n$ et $a, b_{1}, \ldots, b_{n} \in \mathbf{A}$ alors il existe $x_{1}, \ldots, x_{n} \in \mathbf{A}$ tels que $\mathrm{D}_{\mathbf{A}}\left(a, b_{1}, \ldots, b_{n}\right)=\mathrm{D}_{\mathbf{A}}\left(b_{1}+a x_{1}, \ldots, b_{n}+a x_{n}\right)$.

2. En conséquence, si $a_{1}, \ldots, a_{r}, b_{1}, \ldots, b_{n} \in \mathbf{A}$ et $\operatorname{Hdim}\left(\mathbf{A}\left[a_{i}^{-1}\right]\right)<n$ pour $i=1, \ldots, r$ alors il existe $y_{1}, \ldots, y_{n} \in\left\langle a_{1}, \ldots, a_{r}\right\rangle$ tels que

$\mathrm{D}_{\mathbf{A}}\left(a_{1}, \ldots, a_{r}, b_{1}, \ldots, b_{n}\right)=\mathrm{D}_{\mathbf{A}}\left(b_{1}+y_{1}, \ldots, b_{n}+y_{n}\right)$.

Démonstration. - Le premier point est une conséquence directe du lemme 5.7 en faisant $a=b$.

Le deuxième point s'en déduit par récurrence sur $r$ :

$$
\mathfrak{J}=\mathrm{D}_{\mathbf{A}}\left(a_{1}, \ldots, a_{r}, b_{1}, \ldots, b_{n}\right)=\mathrm{D}_{\mathbf{A}}\left(a_{1}, \ldots, a_{r-1}, b_{1}, \ldots, b_{n}\right) \vee \mathrm{D}_{\mathbf{A}}\left(a_{r}\right)=\mathfrak{I} \vee \mathrm{D}_{\mathbf{A}}\left(a_{r}\right),
$$

$\mathfrak{I}=\mathrm{D}_{\mathbf{A}}\left(b_{1}+z_{1}, \ldots, b_{n}+z_{n}\right)$ avec $z_{1}, \ldots, z_{n} \in\left\langle a_{1}, \ldots, a_{r-1}\right\rangle$, donc $\mathfrak{J}=\mathrm{D}_{\mathbf{A}}\left(a_{r}, b_{1}+z_{1}, \ldots, b_{n}+z_{n}\right)$ et on applique une nouvelle fois le résultat.

Remarque. - Dans le cas d'un anneau local nothérien $\mathbf{A}$, l'idéal maximal est radicalement engendré par $n=\mathrm{Kdim} \mathbf{A}$ éléments, mais pas moins. Ceci montre que parmi $n$ «énérateurs radicaux $»$ du maximal, il n'y en a aucun vérifiant $\operatorname{Hdim}\left(\mathbf{A}\left[x^{-1}\right]\right)<n-1 . P$ ar ailleurs $\operatorname{Hdim}(\mathbf{A})=0$ puisque $\mathbf{A}$ est local.

\section{Le splitting off de Serre et le théorème de Forster, à la Heitmann}

Dans cette section nous donnons une version constructive du travail de Heitmann [15] concernant le splitting off de Serre, le théorème de Forster et une variation sur le théorème de Swan. Nous remplaçons la Jdim par la Hdim, qui est plus facile à manipuler et donne des résultats a priori plus précis.

Dans la section suivante, nous donnerons des théorèmes qui sont « meilleurs » dans la mesure où ils sont plus faciles à mettre en œuvre (l'hypothèse est moins lourde car elle ne fait pas intervenir la dimension de nombreux localisés) et où la dimension de Heitmann est mal controlée lors d'une localisation.

\subsection{Manipulations élémentaires de colonnes. -}

Lemme 6.1. - Si $a \in \mathbf{A}, \operatorname{Hdim}\left(\mathbf{A}\left[a^{-1}\right]\right)<n$ et $L \in \mathbf{A}^{n}$ il existe $X \in \mathbf{A}^{n}$ tel que $a \in \mathrm{D}_{\mathbf{A}}(L-a X)$. En outre nous pouvons prendre $X=a Y$ avec $Y \in \mathbf{A}^{n}$.

Démonstration. — Cas particulier du lemme 5.7 : faire $a=b$.

Pour $n=1$ cela donne : $\operatorname{si} \operatorname{Hdim}\left(\mathbf{A}\left[a^{-1}\right]\right) \leq 0$ alors pour tout $b$ on peut trouver $x$ tel que $a \in \mathrm{D}_{\mathbf{A}}(b+x a)$.

Corollaire 6.2. - Soit $M$ une matrice dans $\mathbf{A}^{n \times n}$ et $\delta$ son déterminant. Si $\operatorname{Hdim}\left(\mathbf{A}\left[\delta^{-1}\right]\right)<n$ alors pour tout $C \in \mathbf{A}^{n}$ il existe $X \in \mathbf{A}^{n}$ tel que $\delta \in \mathrm{D}_{\mathbf{A}}(M X-C)$. En outre nous pouvons trouver $X$ de la forme $\delta Y, Y \in \mathbf{A}^{n}$.

Démonstration. — La preuve est basée sur les formules de Cramer. Soit $\widetilde{M}$ la matrice adjointe de $M$, et $L=\widetilde{M} C$. Nous avons $\widetilde{M}(M X-C)=\delta X-L$ pour un vecteur colonne arbitraire $X \in \mathbf{A}^{n}$. Donc l'idéal engendré par les coordonnées de $\delta X-L$ est inclus dans celui engendré par les coordonnées de $M X-C$, et

$$
\mathrm{D}_{\mathbf{A}}(\delta X-L) \leq \mathrm{D}_{\mathbf{A}}(M X-C)
$$


D'après le lemme 6.1 nous pouvons trouver un $X \in \mathbf{A}^{n}$ tel que $\delta \in \mathrm{D}_{\mathbf{A}}(\delta X-L)$, et donc $\delta \in$ $\mathrm{D}_{\mathbf{A}}(M X-C)$ comme demandé.

Pour la fin de cette section nous fixons les notations suivantes :

Notation 6.3. - Soit $F$ une matrice dans $\mathbf{A}^{n \times(p+1)}$ de colonnes $C_{0}, C_{1}, \ldots, C_{p}$, et $G$ la matrice ayant pour colonnes $C_{1}, C_{2}, \ldots, C_{p}$. Soit $\Delta_{k}(F)$ l'idéal déterminantiel d'ordre $k$ de $F$ (l'idéal engendré par les mineurs $\nu$ d'ordre $k$ de $F$ ).

Théorème 6.4. - Fixons $0<k \leq$ p. Supposons que pour chaque mineur $\nu$ d'ordre $k$ de $G$ l'anneau $\mathbf{A}\left[\nu^{-1}\right]$ est de dimension de Heitmann $<k$. Alors il existe $t_{1}, \ldots, t_{p}$ tels que

$$
\mathrm{D}_{\mathbf{A}}\left(C_{0}, \Delta_{k}(F)\right) \leq \mathrm{D}_{\mathbf{A}}\left(C_{0}+t_{1} C_{1}+\cdots+t_{p} C_{p}\right)
$$

(i.e., $\Delta_{k}(F) \subseteq \mathrm{D}_{\mathbf{A}}\left(C_{0}+t_{1} C_{1}+\cdots+t_{p} C_{p}\right)$ et $\mathrm{D}_{\mathbf{A}}\left(C_{0}\right) \leq \mathrm{D}_{\mathbf{A}}\left(C_{0}+t_{1} C_{1}+\cdots+t_{p} C_{p}\right)$ ).

Démonstration. - L'inclusion $\Delta_{k}(F) \subseteq \mathrm{D}_{\mathbf{A}}\left(C_{0}+t_{1} C_{1}+\cdots+t_{p} C_{p}\right)=\mathrm{D}_{\mathbf{A}}\left(C_{0}^{\prime}\right)$ signifie que pour tout mineur $\nu$ d'ordre $k$ de $F$ on a $\nu \in \mathrm{D}_{\mathbf{A}}\left(C_{0}^{\prime}\right)$. Soit $\Delta_{k}(G)$ l'idéal déterminantiel d'ordre $k$ de $G$. Remarquons que si le mineur $\nu$ fait intervenir la colonne $C_{0}$ et les colonnes $C_{i_{j}}\left(1 \leq i_{1}<\cdots<\right.$ $i_{k-1} \leq p$ ) alors on peut y remplacer la colonne $C_{0}$ par la colonne $C_{0}^{\prime}=C_{0}+t_{1} C_{1}+\cdots+t_{p} C_{p}$ sans modifier sa valeur autrement qu'en ajoutant une combinaison linéaire de mineurs d'ordre $k$ de $G$, de sorte que $\nu \in \mathrm{D}_{\mathbf{A}}\left(C_{0}^{\prime}, \Delta_{k}(G)\right)$. En conclusion il nous suffit de réaliser $\Delta_{k}(G) \subseteq \mathrm{D}_{\mathbf{A}}\left(C_{0}^{\prime}\right)$ pour avoir $\Delta_{k}(F) \subseteq \mathrm{D}_{\mathbf{A}}\left(C_{0}^{\prime}\right)$.

En fait il suffit de savoir réaliser

$$
\mathrm{D}_{\mathbf{A}}\left(C_{0}, \nu_{1}\right) \leq \mathrm{D}_{\mathbf{A}}\left(C_{0}+t_{1} C_{1}+\cdots+t_{p} C_{p}\right)=\mathrm{D}_{\mathbf{A}}\left(C_{0}^{\prime}\right)
$$

pour un mineur $\nu_{1}$ d'ordre $k$ de $G$. Car alors nous remplaçons $C_{0}$ par $C_{0}^{\prime}$ dans $F$ (ce qui ne change pas $G$ ) et nous pouvons passer à un autre mineur $\nu_{2}$ de $G$ pour lequel nous obtiendrons $t_{1}^{\prime}, \ldots, t_{p}^{\prime}$ avec

$$
\mathrm{D}_{\mathbf{A}}\left(C_{0}, \nu_{1}, \nu_{2}\right) \leq \mathrm{D}_{\mathbf{A}}\left(C_{0}^{\prime}, \nu_{2}\right) \leq \mathrm{D}_{\mathbf{A}}\left(C_{0}^{\prime}+t_{1}^{\prime} C_{1}+\cdots+t_{p}^{\prime} C_{p}\right)=\mathrm{D}_{\mathbf{A}}\left(C_{0}^{\prime \prime}\right)
$$

avec $C_{0}^{\prime \prime}=C_{0}+t_{1}^{\prime \prime} C_{1}+\cdots+t_{p}^{\prime \prime} C_{p}$ et ainsi de suite.

Pour réaliser

$$
\nu \in \mathrm{D}_{\mathbf{A}}\left(C_{0}+t_{1} C_{1}+\cdots+t_{p} C_{p}\right) \quad \text { et } \quad \mathrm{D}_{\mathbf{A}}\left(C_{0}\right) \leq \mathrm{D}_{\mathbf{A}}\left(C_{0}+t_{1} C_{1}+\cdots+t_{p} C_{p}\right)
$$

pour un mineur $\nu$ d'ordre $k$ de $G$ nous utilisons le corollaire 6.2 : nous trouvons $t_{1}, \ldots, t_{p} \in \nu \mathbf{A}$ (avec $t_{i}=0$ pour les colonnes qui n’interviennent pas dans le mineur $\nu$ ) tels que

$$
\nu \in \mathrm{D}_{\mathbf{A}}\left(C_{0}+t_{1} C_{1}+\cdots+t_{p} C_{p}\right)=\mathrm{D}_{\mathbf{A}}\left(C_{0}^{\prime}\right)
$$

Puisque $t_{1}, \ldots, t_{p}$ sont des multiples de $\nu$ nous avons aussi $\mathrm{D}_{\mathbf{A}}\left(C_{0}\right) \leq \mathrm{D}_{\mathbf{A}}\left(\nu, C_{0}^{\prime}\right)=\mathrm{D}_{\mathbf{A}}\left(C_{0}^{\prime}\right)$ comme demandé.

Toujours avec les notations 6.3, nous obtenons le corollaire suivant.

Théorème 6.5. - Supposons que $1 \in \Delta_{1}(F)$ et que pour $k=1, \ldots, p$ et chaque mineur $\nu$ d'ordre $k$ de $G$ l'anneau $\mathbf{A}\left[\nu^{-1}\right] / \Delta_{k+1}(F)$ est de dimension de Heitmann $<k$. Alors il existe $t_{1}, \ldots, t_{p}$ tels que le vecteur $C_{0}+t_{1} C_{1}+\cdots+t_{p} C_{p}$ est unimodulaire.

En particulier si $k \leq p, 1 \in \Delta_{k}(F)$ et $\operatorname{Hdim}\left(\mathbf{A}\left[\nu^{-1}\right]\right)<k$ pour tout $\nu \in \mathbf{A}$, il existe $t_{1}, \ldots, t_{p}$ tels que le vecteur $C_{0}+t_{1} C_{1}+\cdots+t_{p} C_{p}$ est unimodulaire.

Démonstration. - En utilisant le théorème 6.4, nous définissons une suite de vecteurs $C_{0}^{k}, k=$ $1, \ldots, p$ avec $C_{0}^{1}=C_{0}$. Pour $k=1, \ldots, p$, nous raisonnons dans $\mathbf{A}_{k}=\mathbf{A} / \Delta_{k+1}(F)$. Nous construisons $C_{0}^{k+1}$ de la forme $C_{0}^{k}+u_{1}^{k} C_{1}+\cdots+u_{p}^{k} C_{p}$ tel que $\mathrm{D}_{\mathbf{A}_{k}}\left(C_{0}^{k}, \Delta_{k}(F)\right) \leq \mathrm{D}_{\mathbf{A}_{k}}\left(C_{0}^{k+1}\right)$. Cela signifie que nous avons, dans $\mathbf{A}$

$$
\mathrm{D}_{\mathbf{A}}\left(C_{0}^{k}, \Delta_{k}(F)\right) \leq \mathrm{D}_{\mathbf{A}}\left(C_{0}^{k+1}, \Delta_{k+1}(F)\right)
$$

D'où le résultat puisque $\Delta_{1}(F)=1$ et $\Delta_{p+1}(F)=0$. 
Un cas particulier plus simple s'énonce avec la dimension de Krull. Notez que la matrice $G$ ne figure plus dans l'hypothèse. La conclusion resterait donc valable après avoir permuté des colonnes.

Théorème 6.6. - Supposons que $1=\Delta_{1}(F)$ et que pour $k=1, \ldots, p$, l'anneau $\mathbf{A} / \Delta_{k+1}(F)$ est de dimension de Krull $<k$. Alors il existe $t_{1}, \ldots, t_{p}$ tels que le vecteur $C_{0}+t_{1} C_{1}+\cdots+t_{p} C_{p}$ est unimodulaire.

En particulier si $\operatorname{Kdim} \mathbf{A}<k \leq p$ et $\Delta_{k}(F)=1$, il existe un vecteur unimodulaire dans le module image de $F$.

Notez que la condition $1 \in \Delta_{1}(F)$ peut se relire « $\mathbf{A} / \Delta_{1}(F)$ est de dimension de Krull $<0 »$. On aurait donc pu énoncer l'hypothèse du théorème sous la forme : pour chaque $k \geq 0$ l'anneau A $/ \Delta_{k+1}(F)$ est de dimension de Krull $<k$.

Nous donnons maintenant en mathématiques classiques une reformulation « avec idéaux premiers $»$ du théorème 6.5 dans lequel on remplace la dimension de Heitmann par la dimension de Krull. Dans l'énoncé qui suit, $\mathrm{D}_{\mathbf{A}}(\mathfrak{J})$ pour un idéal de type fini $\mathfrak{J}$ est identifié à l'ouvert quasicompact correspondant de $\operatorname{Spec} \mathbf{A}$ et $V_{\mathbf{A}}(\mathfrak{J})$ est son complémentaire.

Théorème 6.7. - Pour tout idéal premier $\mathfrak{p}$ notons $r_{\mathfrak{p}}$ le rang de la matrice $F$ vue dans le corps des fractions de $\mathbf{A} / \mathfrak{p}$ (on a $0 \leq r_{\mathfrak{p}} \leq p+1$ ). On donne deux formulations équivalentes pour les hypothèses du théorème.

1. Supposons que pour $k=0, \ldots, p$ la dimension de Krull du sous-espace spectral $\mathrm{D}_{\mathbf{A}}\left(\Delta_{k}(G)\right) \cap$ $\mathrm{V}_{\mathbf{A}}\left(\Delta_{k+1}(F)\right)$ de $\operatorname{Spec} \mathbf{A}$ soit $<k$ (pour $k=0$ cela signifie simplement $1 \in \Delta_{1}(F)$ ).

2. Supposons que pour tout $\mathfrak{p} \in \mathrm{D}_{\mathbf{A}}\left(\Delta_{r_{\mathfrak{p}}-1}(G)\right)$ on a $\operatorname{Kdim}(A / \mathfrak{p})<r_{\mathfrak{p}}$.

Alors il existe un vecteur unimodulaire dans le module image de $F$. En outre ce vecteur peut être écrit sous la forme donnée dans le théorème 6.5.

Démonstration. - Fixons $k \leq p$ et posons $\mathbf{B}_{k}=\mathbf{A} / \Delta_{k+1}(F)$. Soit $\nu$ un mineur d'ordre $k$ de $G$. Les idéaux premiers de $\mathbf{B}_{k}[1 / \nu]$ peuvent être identifiés aux idéaux premiers $\mathfrak{p}$ de $\mathbf{A}$ qui contiennent $\Delta_{k+1}(F)$ mais ne contiennent pas $\nu$. Donc le maximum $\operatorname{des} \mathbf{K} \operatorname{dim} \mathbf{B}_{k}[1 / \nu]$ est la longueur maximum d'une chaîne d'idéaux premiers qui contiennent $\Delta_{k+1}(F)$ mais évitent au moins un mineur d'ordre $k$ de $G$. C'est donc la dimension de Krull de $\mathrm{D}_{\mathbf{A}}\left(\Delta_{k}(G)\right) \cap \mathrm{V}_{\mathbf{A}}\left(\Delta_{k+1}(F)\right)$. Ainsi la première formulation de l'hypothèse nous ramène aux hypothèses du théorème 6.5 .

Soit $\mathfrak{p}$ un idéal premier de A. Dire que $r_{\mathfrak{p}} \leq k$ signifie que modulo $\mathfrak{p}$ tous les mineurs d'ordre $p+1$ sont nuls, c'est-à-dire $\Delta_{k+1}(F) \subseteq \mathfrak{p}$. La deuxième condition est donc bien équivalente à la première.

\subsection{Quelques conséquences. -}

Le théorème splitting off de Serre, non nœethérien. - On déduit directement du théorème 6.5 , comme dans $[\mathbf{1 0}, \mathbf{1 5}]$, la version suivante « améliorée » du Splitting-off de Serre.

Théorème 6.8. - (théorème de Serre non nœethérien à la Heitmann, version dimension de Krull) Soit $M$ un $\mathbf{A}$-module projectif de rang $\geq k$ sur un anneau $\mathbf{A}$ tel que $\operatorname{Kdim} \mathbf{A}<k$. Alors $M \simeq N \oplus \mathbf{A}$ pour un certain $N$.

Démonstration. - Si $F$ est une matrice de projection dont l'image est (isomorphe à) $M$, l'hypothèse que $M$ est de rang $\geq k$ signifie que $\Delta_{k}(F)=\langle 1\rangle$. Le théorème 6.5 nous donne dans l'image de $F$ un vecteur unimodulaire. C'est un élément $C$ de $M$ tel que $\mathbf{A} C$ est facteur direct dans $M$.

Remarque. - Dans le fonctionnement de cette preuve, on voit qu'on aurait pu se contenter de supposer que $M$ est un module image d'une matrice $F$ telle que $\Delta_{k}(F)=1$. 
Le théorème de Forster, versions non noethériennes. - Voici une version du théorème de Forster [13].

Théorème 6.9. - Soit $M$ un module de type fini sur un anneau $\mathbf{A}$ et $\mathfrak{J}$ l'annulateur de $M$. Si la dimension de Krull de $\mathbf{A} / \mathfrak{J}$ est $\leq d$. et si $M$ est localement engendré par $r$ éléments alors $M$ peut être engendré par $d+r$ éléments.

Démonstration. - $M$ est un quotient d'un module de présentation finie $M^{\prime}$ dont l'idéal de Fitting d'ordre $r$ égal à 1 , et nous pouvons donc supposer $M$ de présentation finie. On regarde $M$ comme un A/ $\mathfrak{J}$-module. Soit $m_{0}, m_{1}, \ldots, m_{p}$ un système de générateurs de $M$ et $F$ une matrice de présentation de $M$ correspondant à ce système générateur. Dire que $M$ est localement engendré par $r$ éléments signifie que $1=\Delta_{p+1-r}(F)$. Si $p \geq d+r$ on a $1=\Delta_{d+1}(F)$ et d'après le théorème 6.4 , appliqué à la matrice transposée de $F$, nous pouvons trouver $t_{1}, \ldots, t_{p}$ tels que $M$ soit engendré par $m_{1}-t_{1} m_{0}, \ldots, m_{p}-t_{p} m_{0}$.

Dans le cas d'un module de présentation finie le théorème 6.5 appliqué à une matrice de présentation du module nous donne des énoncés plus sophistiqués comme dans [15].

Donnons par exemple une formulation avec la dimension de Krull, plus facile à formuler.

Théorème 6.10. - (de Forster, version non nœthérienne de Heitmann)

Soit $M$ un module de présentation finie sur $\mathbf{A}$. Notons $\mathfrak{f}_{k}$ son idéal de Fitting d'ordre $k$ et $V_{k}=$ $\mathrm{V}_{\mathbf{A}}\left(\mathfrak{f}_{k}\right) \subseteq \operatorname{Spec} \mathbf{A}$.

- Version constructive (sans points). Si $\operatorname{Kdim}\left(\mathbf{A}\left[\nu^{-1}\right] / \mathfrak{f}_{k}\right)<m-k$ pour $k=0, \ldots, m$ et pour tout générateur $\nu$ de $\mathfrak{f}_{k+1}$, alors $M$ peut être engendré par $m$ éléments.

- Version classique (avec points). Pour tout idéal premier $\mathfrak{p}$ de $\mathbf{A}$, notons $\mu_{\mathfrak{p}}$ la dimension de l'espace vectoriel $M \otimes_{\mathbf{A}} \operatorname{Frac}(\mathbf{A} / \mathfrak{p})$ sur le corps $\operatorname{Frac}(\mathbf{A} / \mathfrak{p})$ (autrement dit $\mu_{\mathfrak{p}}=k$ si et seulement si $\left.\mathfrak{p} \in V_{k-1} \backslash V_{k}\right)$.

- Si pour $k=0, \ldots, m$ et pour tout $\mathfrak{p} \in V_{k} \backslash V_{k+1}$ on a $\operatorname{Kdim}(\mathbf{A} / \mathfrak{p}) \leq m-k$, ou ce qui revient au même,

- si pour tout $\mathfrak{p} \in V_{0}$ on a $\operatorname{Kdim}(\mathbf{A} / \mathfrak{p})+\mu_{\mathfrak{p}} \leq m$,

alors $M$ peut être engendré par $m$ éléments.

Rappelons que le support du module $M$ est le fermé $V_{0}$. La version avec points peut donc être énoncée en demandant que pour tout $\mathfrak{p}$ dans le support de $M$ on ait $\operatorname{Kdim}(\mathbf{A} / \mathfrak{p})+\mu_{\mathfrak{p}} \leq m$.

La version sans points reste valable en remplaçant la dimension de Krull par celle de Heitmann, mais pas la version avec points, car on n'obtient pas alors l'équivalent de la version sans points.

La version sans points avec la Jdim (reformulée avec points) est la variante du théorème de Swan obtenue par Heitmann dans [15].

\section{Théorèmes de Serre et de Swan, non nœthériens}

Nous passons maintenant à des versions où la dimension de Heitmann qui intervient n'utilise plus de localisations.

Ceci nous permet d'améliorer le théorème de Swan lui-même [24], répondant ainsi positivement à une question posée dans [15].

\subsection{Manipulations élémentaires de colonnes. -}

Lemme 7.1. - Soient $L, L_{1} \ldots, L_{k} \in \mathbf{A}^{m}$ et $b_{1}, \ldots, b_{k} \in A$. Si $\operatorname{Hdim}(\mathbf{A})<k$ et

$$
1=\mathrm{D}_{\mathbf{A}}\left(a, b_{1}, \ldots, b_{k}\right) \vee \mathrm{D}_{\mathbf{A}}(L)
$$

alors il existe $x_{1}, \ldots, x_{k} \in a \mathbf{A}$ tels que

$$
1=\mathrm{D}_{\mathbf{A}}\left(b_{1}+a x_{1}, \ldots, b_{k}+a x_{k}\right) \vee \mathrm{D}_{\mathbf{A}}\left(L+x_{1} L_{1}+\cdots+x_{k} L_{k}\right)
$$


Démonstration. - La preuve est par induction sur $k$. Pour $k=0$, c'est clair. Si $k>0$, soit $\mathfrak{j}=\mathrm{H}_{\mathbf{A}}\left(b_{k}\right)$ l'idéal bord de Heitmann $b_{k}$. On a $b_{k} \in \mathfrak{j}$ et $\operatorname{Hdim}(\mathbf{A} / \mathfrak{j})<k-1$, donc par induction, on peut trouver $y_{1}, \ldots, y_{k-1}$ tels que

$$
1=\mathrm{D}_{\mathbf{A} / \mathfrak{j}}\left(b_{1}+a^{2} y_{1}, \ldots, b_{k-1}+a^{2} y_{k-1}, L+a y_{1} L_{1}+\cdots+a y_{k-1} L_{k-1}\right)
$$

Posons $L^{\prime}=L+a y_{1} L_{1}+\cdots+a y_{k-1} L_{k-1}, X=\left(b_{1}+a^{2} y_{1}, \ldots, b_{k-1}+a^{2} y_{k-1}\right)$ et remarquons que $\mathrm{D}_{\mathbf{A}}\left(L^{\prime}, a\right)=\mathrm{D}_{\mathbf{A}}(L, a)$ et $\mathrm{D}_{\mathbf{A}}(X, a)=\mathrm{D}_{\mathbf{A}}\left(a, b_{1}, \ldots b_{k-1}\right)$. L'égalité $(\alpha)$ signifie qu'il existe $y_{k}$ tel que $b_{k} y_{k} \in \mathrm{J}_{\mathbf{A}}(0)$ et

$$
1=\mathrm{D}_{\mathbf{A}}(X) \vee \mathrm{D}_{\mathbf{A}}\left(L^{\prime}\right) \vee \mathrm{D}_{\mathbf{A}}\left(b_{k}, y_{k}\right)
$$

On a

$$
\mathrm{D}_{\mathbf{A}}\left(X, L^{\prime}+a y_{k} L_{k}\right) \vee \mathrm{D}_{\mathbf{A}}\left(b_{k}, a^{2}\right)=\mathrm{D}_{\mathbf{A}}\left(a, b_{1}, \ldots, b_{k}, L\right)=1
$$

et, d'après $(\beta)$,

$$
\mathrm{D}_{\mathbf{A}}\left(X, L^{\prime}+a y_{k} L_{k}\right) \vee \mathrm{D}_{\mathbf{A}}\left(b_{k}, y_{k}\right)=\mathrm{D}_{\mathbf{A}}\left(X, L^{\prime}\right) \vee \mathrm{D}_{\mathbf{A}}\left(b_{k}, y_{k}\right)=1
$$

$(\gamma)$ et $(\delta)$ impliquent par distributivité

$$
\mathrm{D}_{\mathbf{A}}\left(X, L^{\prime}+a y_{k} L_{k}, b_{k}, a^{2} y_{k}\right)=1=\mathrm{J}_{\mathbf{A}}\left(X, L^{\prime}+a y_{k} L_{k}, b_{k}, a^{2} y_{k}\right)
$$

et d'après le lemme 5.1 puique $b_{k} a^{2} y_{k} \in \mathrm{J}_{\mathbf{A}}(0)$

$$
1=\mathrm{J}_{\mathbf{A}}\left(X, L^{\prime}+a y_{k} L_{k}, b_{k}+a^{2} y_{k}\right)
$$

ce qui est exactement le résultat souhaité (34) avec $x_{i}=a y_{i}$.

Remarques. -

1) Lorsqu'on remplace la dimension de Heitmann par celle de Krull, le lemme précédent admet une version « uniforme » dans laquelle les $x_{i}$ ne dépendent pas de $a$ (comme dans le lemme 5.2).

2) Le lemme ci-dessus peut être vu comme une variante raffinée du théorème 5.4. En fait dans la suite, nous utilisons ce lemme uniquement avec l'hypothèse renforcée comme suit : $\operatorname{Si} \operatorname{Hdim}(\mathbf{A})<k$ et $1=\mathrm{D}_{\mathbf{A}}(a, L) \ldots$

On en déduit le corollaire suivant (qui simplifie l'argument dans [5] et a été suggéré par Lionel $\operatorname{Ducos}[\mathbf{9}])$.

Corollaire 7.2. - Supposons que $C, C_{1}, \ldots, C_{k}$ sont des vecteurs dans $\mathbf{A}^{m}$ et $\nu$ est un mineur d'ordre $k$ de $C_{1}, \ldots, C_{k}$. Si $1=\mathrm{D}_{\mathbf{A}}(\nu) \vee \mathrm{D}_{\mathbf{A}}(C)$ et $\operatorname{Hdim}(\mathbf{A})<k$ alors il existe $x_{1}, \ldots, x_{k} \in A$ tels que $1=\mathrm{D}_{\mathbf{A}}\left(C+x_{1} C_{1}+\cdots+x_{k} C_{k}\right)$.

Démonstration. - On prend $a=\nu$ et $b_{i}$ le mineur obtenu en remplaçant $C_{i}$ par $C$ dans $C_{1}, \ldots, C_{k}$. On applique le lemme 7.1. On remarque alors que $b_{i}+a x_{i}$ est aussi le mineur obtenu en remplaçant $C_{i}$ par $C+x_{1} C_{1}+\cdots+x_{n} C_{n}$ dans $C_{1}, \ldots, C_{n}$ de sorte que $b_{i}+a x_{i} \in \mathrm{D}_{\mathbf{A}}\left(C+x_{1} C_{1}+\cdots+x_{n} C_{n}\right)$ et

$$
1=\mathrm{D}_{\mathbf{A}}\left(b_{1}+a x_{1}, \ldots, b_{n}+a x_{n}\right) \vee \mathrm{D}_{\mathbf{A}}\left(C+x_{1} C_{1}+\cdots+x_{n} C_{n}\right)
$$

implique que $\mathrm{D}_{\mathbf{A}}\left(C+x_{1} C_{1}+\cdots+x_{n} C_{n}\right)=1$.

Nous allons prouver une variante du théorème 6.4. Nous reprenons les notations 6.3.

Lemme 7.3. - Si $\mathrm{D}_{\mathbf{A}}(C) \vee \Delta_{k}(G)=1$ et $\operatorname{Hdim}(\mathbf{A})<k$ alors il existe $t_{1}, \ldots, t_{p}$ tels que $C+$ $t_{1} C_{1}+\cdots+t_{p} C_{p}$ est unimodulaire.

Démonstration. - Comme $\mathrm{D}_{\mathbf{A}}(C) \vee \Delta_{k}(G)=1$, on a une famille $\nu_{1}, \ldots, \nu_{p}$ de mineurs de $G$ d'ordre $k$ tels que $1=\mathrm{D}_{\mathbf{A}}(C) \vee \bigvee_{i} \mathrm{D}_{\mathbf{A}}\left(\nu_{i}\right)$. On applique alors le corollaire 7.2 pour avoir $\mathrm{D}_{\mathbf{A}}(C) \vee \mathrm{D}_{\mathbf{A}}\left(\nu_{k}\right)=1$ dans $\mathbf{A} / J_{k}$ avec $J_{k}=\bigvee_{i>k} \mathrm{D}_{\mathbf{A}}\left(\nu_{i}\right)$ jusqu'à ce que nous obtenions $\mathrm{D}_{\mathbf{A}}(C)=1$ dans $\mathbf{A}$.

Théorème 7.4. - Fixons $k \leq p$. Supposons que la dimension de Heitmann de $\mathbf{A}$ est $<k$ et $\Delta_{k}(F)=1$. Alors il existe $t_{1}, \ldots, t_{p}$ tels que $1=\mathrm{D}_{\mathbf{A}}\left(C_{0}+t_{1} C_{1}+\cdots+t_{p} C_{p}\right)$.

Démonstration. — Cela résulte directement du lemme 7.3. 
Les théorèmes de Serre et Swan avec la dimension de Heitmann. - On a alors les résultats suivants, que l'on prouve à partir du théorème 7.4 par les mêmes arguments que pour les théorèmes 6.8 et 6.9 .

Théorème 7.5. — (théorème de Serre, version dimension de Heitmann)

Soit $M$ un A-module projectif de rang $\geq k$ sur un anneau $\mathbf{A}$ tel que $\operatorname{Hdim} \mathbf{A}<k$. Alors $M \simeq N \oplus \mathbf{A}$ pour un certain $N$.

Théorème 7.6. - (théorème de Swan, version dimension de Heitmann)

Si $\operatorname{Hdim}(\mathbf{A}) \leq d$ et si $M$ est un module de type fini sur $\mathbf{A}$ localement engendré par $r$ éléments, alors $M$ peut être engendré par $d+r$ éléments.

\subsection{D'autres résultats. -}

Le théorème de Swan, forme sophistiquée. — Un raffinement du théorème 7.4 est le résultat suivant.

Théorème 7.7. - Supposons que $1 \in \Delta_{1}(F)$ et que pour $k=1, \ldots, p$, l'anneau $\mathbf{A} / \Delta_{k+1}(F)$ est de dimension de Heitmann $<k$. Alors il existe $t_{1}, \ldots, t_{p}$ tels que le vecteur $C_{0}+t_{1} C_{1}+\cdots+t_{p} C_{p}$ est unimodulaire.

Démonstration. - On applique le lemme 7.3 successivement avec les anneaux $\mathbf{A} / \Delta_{2}(F), \mathbf{A} / \Delta_{3}(F)$, ... (raisonner comme dans la preuve du théorème 6.5).

Théorème 7.8. - (théorème de Swan général, version dimension de Heitmann)

Soit $M$ un module de type fini sur $\mathbf{A}$ et $\mathfrak{f}_{k}$ son idéal de Fitting d'ordre $k$. Si la dimension $\operatorname{Hdim}\left(\mathbf{A} / \mathfrak{f}_{k}\right)$ est strictement inférieure à $m-k$ pour $k=0, \ldots, m$, alors $M$ peut être engendré par $m$ éléments.

Démonstration. — Si le module est de présentation finie il suffit d'appliquer le théorème 7.7 à la matrice transposée d'une matrice de présentation de $M$ (de la même manière que pour le théorème 6.10).

Le raisonnement dans le cas général est un peu plus subtil. Notons $h_{1}, \ldots, h_{q}$ des générateurs de $M$ et supposons $q>m$. Notons $h=\left(h_{1}, \ldots, h_{q}\right)$. Toute relation entre les $h_{i}$ peut être écrite comme un vecteur colonne $C \in \mathbf{A}^{q}$ qui vérifie $h C=0$. L'idéal de Fitting $\mathfrak{f}_{q-i}$ de $M$ est l'idéal $\Delta_{i}$ engendré par les mineurs d'ordre $i$ des matrices $F \in \mathbf{A}^{q \times n}$ qui vérifient $h F=0$ (c'est-à-dire les matrices dont les colonnes sont des relations entre les $h_{i}$ ). Nous les appelerons des « matrices de relation $»$. Comme $\mathrm{D}_{\mathbf{A}}\left(\Delta_{q}\right)=\mathrm{D}_{\mathbf{A}}\left(\mathfrak{f}_{0}\right)=\mathrm{D}_{\mathbf{A}}(\operatorname{Ann}(M))$ et que nous pouvons remplacer $\mathbf{A}$ par $\mathbf{A} / \operatorname{Ann}(M)$, nous supposons sans perte de généralité que $\mathrm{D}_{\mathbf{A}}\left(\Delta_{q}\right)=\mathrm{D}_{\mathbf{A}}(0)$.

Les hypothèses impliquent que $\operatorname{Hdim}\left(\mathbf{A} / \Delta_{k}\right)<k-1$ pour $k=1, \ldots, q$ (ce sont les hypothèses lorsque $q=m+1)$.

On a donc $\Delta_{1}=1$, ce qui se constate sur une matrice de relations $F$. On peut alors appliquer le lemme 7.3 avec la matrice $F$, l'entier $k=1$ et l'anneau $\mathbf{A} / \Delta_{2}$. Ceci nous donne une relation $C$ telle que $\mathrm{D}_{\mathbf{A} / \Delta_{2}}(C)=1$, c'est-à-dire encore telle qu'il existe $a \in \Delta_{2}$ vérifiant $1+a \in \mathrm{D}_{\mathbf{A}}(C)$.

Le fait que $a \in \Delta_{2}$ se constate sur une matrice de relations $G$ et on considère la nouvelle matrice $F=(C \mid G)$. On a donc $\mathrm{D}_{\mathbf{A}}(C) \vee \Delta_{2}(G)=1$ et $\operatorname{Hdim}\left(\mathbf{A} / \Delta_{3}\right)<2$. On peut alors appliquer le lemme 7.3 avec la nouvelle matrice $F$, l'entier $k=2$ et l'anneau $\mathbf{A} / \Delta_{3}$. Ceci nous donne une relation $C$ telle que $\mathrm{D}_{\mathbf{A} / \Delta_{3}}(C)=1$, et ainsi de suite.

On obtient en fin de compte une relation $C$ telle que $\mathrm{D}_{\mathbf{A}}(C)=1$, puisque $\mathrm{D}_{\mathbf{A}}\left(\Delta_{q}\right)=0$. Et $\operatorname{Hdim} \mathbf{A}=\operatorname{Hdim}\left(\mathbf{A} / \Delta_{q}\right)<q-1$. En conséquence le corollaire 5.5 du théorème stable range de Bass s'applique et nous pouvons transformer la colonne $C$ en $(1,0, \ldots, 0)$ par des manipulations élémentaires, ce qui revient à remplacer le système générateur $h$ par une autre système de $q$ éléments dont le premier est nul, bref à réduire de 1 le nombre de générateurs. 
Notons qu'il n'y a pas a priori de version « avec points »du théorème précédent car la Hdim n'est pas (à notre connaissance) la dimension de Krull d'un espace spectral. Pour obtenir une version avec points, nous devrions considérer la variante (a priori plus faible) dans laquelle la Hdim est replacée par la Jdim. On aurait alors sans doute une formulation classique utilisant les points du J-spectre de Heitmann.

Le théorème de simplification de Bass, avec la dimension de Heitmann, sans hypothèse nothérienne. - Nous prouvons maintenant le théorème de simplification de Bass.

Lemme 7.9. - Si $\Delta_{k}(G)=1=\mathrm{D}_{\mathbf{A}}(C) \vee \mathrm{D}_{\mathbf{A}}(a)$ et $\operatorname{Hdim}(\mathbf{A})<k$ alors il existe $t_{1}, \ldots, t_{p}$ tels que $1=\mathrm{D}_{\mathbf{A}}\left(C+a t_{1} C_{1}+\cdots+a t_{p} C_{p}\right)$.

Démonstration. - Il suffit d'appliquer le lemme 7.3 aux vecteurs $C, a C_{1}, \ldots, a C_{n}$ (ce qui remplace $G \operatorname{par} a G)$

Théorème 7.10. - (théorème de simplification de Bass, version dimension de Heitmann) Soit $M$ un $\mathbf{A}$-module projectif de rang $\geq k$ sur un anneau $\mathbf{A}$ tel que $\operatorname{Hdim} \mathbf{A}<k$, et $N, Q$ deux autres A-modules projectifs de type fini. Alors $M \oplus Q \simeq N \oplus Q$ implique $M \simeq N$.

Démonstration. - Pour être complet, on redonne l'argument de [10]. On peut supposer que $Q=\mathbf{A}$ et que $N \subseteq \mathbf{A}^{m}$ est image d'une matrice idempotente. On a un isomorphisme $\phi: M \oplus \mathbf{A} \rightarrow N \oplus \mathbf{A} \subseteq$ $\mathbf{A}^{m+1}$. Soit $(C, a) \in \mathbf{A}^{m+1}$ le vecteur $\phi(0,1)$. Il nous faut construire un automorphisme de $N \oplus \mathbf{A}$ qui envoie $(C, a)$ sur $(0,1)$.

Soit $\alpha: M \oplus \mathbf{A} \rightarrow \mathbf{A},(U, x) \mapsto x$. La forme linéaire $\alpha \circ \phi^{-1}$ envoie $(C, a)$ sur 1 , d'où $\mathrm{D}_{\mathbf{A}}(C) \vee \mathrm{D}_{\mathbf{A}}(a)=$ 1. Donc, par le lemme 7.9, il existe $C^{\prime} \in N$ tel que $\mathrm{D}_{\mathbf{A}}\left(C+a C^{\prime}\right)=1$. On a donc une forme linéaire $\lambda: N \rightarrow \mathbf{A}$ vérifiant $\lambda\left(C+a C^{\prime}\right)=1$ (obtenue comme restriction d'une forme linéaire sur $\mathbf{A}^{m}$ ). On a alors les trois automorphismes suivants de $N \oplus \mathbf{A}$ qui p ar composition réalisent le but fixé :

$$
\begin{array}{lllll}
\psi_{1}:(V, x) & \mapsto\left(V+x C^{\prime}, \lambda(x C-a V)\right), & & (C, a) \mapsto\left(C+a C^{\prime}, 0\right) \\
\psi_{2}: & (V, x) & \mapsto(V, x+\lambda(V)), & & \left(C+a C^{\prime}, 0\right) \mapsto\left(C+a C^{\prime}, 1\right) \\
\psi_{3}: & (V, x) & \mapsto\left(V-x\left(C+a C^{\prime}\right), x\right), & & \left(C+a C^{\prime}, 1\right) \mapsto(0,1)
\end{array}
$$

(on vérifie que $\psi_{1}$ est un automorphisme en remarquant que si $\psi_{1}(V, x)=(W, y)$ alors $x=y+$ $a \lambda(W))$.

Remarques. - Dans le fonctionnement de cette preuve, on voit qu'on aurait pu se contenter de supposer que $N$ est un module image d'une matrice $F$ telle que $\Delta_{k}(F)=1$ et $M \oplus Q \simeq N \oplus Q$ pour un module projectif $Q$.

Notons aussi que les automorphismes $\psi_{i}$ sont réalisés en pratique comme des automorphismes de $\mathbf{A}^{n+1}$ qui fixent $N \oplus \mathbf{A}$. De manière plus générale tous les théorèmes d'algèbre commutative que nous avons démontrés se ramènent en fin de compte à des théorèmes concernant les matrices et leurs manipulations élémentaires.

\section{Références}

[1] Banaschewski, B. Radical ideals and coherent frames. Comment. Math. Univ. Carolin. 37 no. 2, (1996), 349-370.

[2] Cederquist J., Coquand T. Entailment relations and Distributive Lattices Logic Colloquium '98 (Prague), 127-139, Lect. Notes Log., 13. Assoc. Symbol. Logic, Urbana, (2000).

[3] Coquand T. Sur un théorème de Kronecker concernant les variétés algébriques C. R. Acad. Sci. Paris, Ser. I 338 (2004), 291-294.

[4] Coquand T., Lombardi H. Hidden constructions in abstract algebra (3) Krull dimension of distributive lattices and commutative rings. dans : Commutative ring theory and applications. Eds : Fontana M., Kabbaj S.-E., Wiegand S. Lecture notes in pure and applied mathematics vol 231. M. Dekker. (2002) 477-499.

[5] Coquand T., Lombardi H., Quitté C. Generating non nœtherian modules constructively. Manuscripta mathematica 115, (2004), 513-520. 
[6] Coquand T., Lombardi H., Roy M.-F. Une caractérisation élémentaire de la dimension de Krull. From Sets and Types to Analysis and Topology : Towards Practicable Foundations for Constructive Mathematics (L. Crosilla, P. Schuster, eds.). Oxford University Press, 2005.

[7] Coquand T., Lombardi H., Schuster P. A nilregular element property. Archiv der Mathematik 85 (2005), 49-54.

[8] Curry, H. B. Foundations of mathematical logic McGraw-Hill Book Co., Inc., New York-San Francisco, Calif.-Toronto-London 1963

[9] Ducos L., Vecteurs unimodulaires et systèmes générateurs. Journal of Algebra 297 (2006), 566-583.

[10] Eisenbud D., Evans E. G., Jr. Generating modules efficiently : theorems from algebraic K-theory. J. Algebra 27 (1973), 278-305.

[11] Eisenbud D., Evans E. G., Jr. Every algebraic set in $n$-space is the intersection of $n$ hypersurfaces. Inventiones math. 19 (1973), 107-112.

[12] Español L. Constructive Krull dimension of lattices. Rev. Acad. Cienc. Zaragoza (2) 37 (1982), 5-9.

[13] Forster O.. Über die Anzahl der Erzeugenden eines Ideals in einem Notherschen Ring. Math. Z. 84 (1964) 80-87.

[14] Heitmann, R. Generating ideals in Prüfer domains. Pacific J. Math. 62 (1976) 117-126.

[15] Heitmann, R. Generating non-Nœtherian modules efficiently. Michigan Math. 312 (1984) 167-180.

[16] Hochster M. Prime ideal structure in commutative rings. Trans. Amer. Math. Soc. 142 (1969) 43-60.

[17] Johnstone P. Stone Spaces. Cambridge Studies in Advanced Mathematics, 3. Cambridge University Press, Cambridge, (1986).

[18] Joyal A. Le théorème de Chevalley-Tarski. Cahiers de Topologie et Géometrie Differentielle, (1975).

[19] Kronecker L. Grundzüge einer arithmetischen Theorie der algebraischen Grössen. J. reine angew. Math. 92, (1882) 1-123. Réimprimé dans Leopold Kronecker's Werke, II, 237-387.

[20] T.Y. Lam. Serre's conjecture. Lecture Notes in Mathematics, Vol. 635. Springer-Verlag, Berlin-New York, 1978.

[21] Lombardi H. Dimension de Krull, Nullstellensätze et Évaluation dynamique. Math. Zeitschrift, 242, (2002), 23-46.

[22] Stone M. H. Topological representations of distributive lattices and Brouwerian logics. Cas. Mat. Fys. 67, (1937), 1-25.

[23] Storch U. Bemerking zu einem Satz van M. Kneser. Arch. Math. 23, (1972), 403-404.

[24] Swan R. G. The Number of Generators of a Module. Math. Z. 102 (1967), 318-322.

[25] Van der Waerden. Review Zentralblatt für Math 24, (1941) 276.

15 novembre 2006

Thierry Coquand, Chalmers, University of Göteborg, Sweden • E-mail : coquand@cs.chalmers.se Url : http://hlombardi.free.fr/

Henri Lombardi, Laboratoire de Mathématiques, UMR CNRS 6623, UFR des Sciences et Techniques, Université de Franche-Comté, 25030 Besançon cedex, France • E-mail : henri.lombardi@univ-fcomte.fr Url : http://hlombardi.free.fr/

Claude Quitté, Laboratoire de Mathématiques,, SP2MI, Boulevard 3, Teleport 2, BP 179,, 86960 Futuroscope Cedex, France $\bullet$ E-mail : quitte@mathlabo.univ-poitiers.fr 